quatrième série-tome 41 fascicule 5 septembre-octobre 2008

$$
\begin{aligned}
& \text { ANNALES } \\
& \text { SCIENTIFIQUES } \\
& \text { de } \\
& \text { L'ECOLE } \\
& \text { NORMALE } \\
& \text { SUPÉRIEURE }
\end{aligned}
$$

Elena MANTOVAN

On non-basic Rapoport-Zink spaces 
Ann. Scient. Éc. Norm. Sup

$4^{\mathrm{e}}$ série, t. 41, 2008, p. 671 à 716

\title{
ON NON-BASIC RAPOPORT-ZINK SPACES
}

\author{
BY ELENA MANTOVAN
}

Abstract. - In this paper we study certain moduli spaces of Barsotti-Tate groups constructed by Rapoport and Zink as local analogues of Shimura varieties. More precisely, given an isogeny class of Barsotti-Tate groups with unramified additional structures, we investigate how the associated (nonbasic) moduli spaces compare to the (basic) moduli spaces associated with its isoclinic constituents.

This aspect of the geometry of the Rapoport-Zink spaces is closely related to Kottwitz's prediction that their $l$-adic cohomology groups provide a realization of certain cases of local Langlands correspondences and in particular to the question of whether they contain any supercuspidal representations.

Our results are compatible with this prediction and identify many cases when no supercuspidal representations appear. In those cases, we prove that the $l$-adic cohomology of the non-basic spaces is equal (in the appropriate sense) to the parabolic induction of the $l$-adic cohomology of some associated lower-dimensional (and in the most favorable cases basic) Rapoport-Zink spaces. Such an equality was originally conjectured by Harris in [11] (Conjecture 5.2, p. 420).

RÉSUMÉ. - Dans cet article, on considère certains espaces de Rapoport-Zink non-ramifiés, associés à des groupes $p$-divisibles non-basiques et on étudie leur géométrie vis-à-vis de celle des espaces de Rapoport-Zink basiques correspondants.

L'origine de ce problème se situe, d'une part, dans la conjecture de Kottwitz concernant la réalisation des correspondances de Langlands locales dans la cohomologie étale $l$-adique des espaces de Rapoport-Zink et, d'autre part, plus simplement dans la question d'identifier pour lesquels de ces espaces la partie supercuspidale de la cohomologie n'est pas vide.

Nos résultats sont compatibles avec cette conjecture et, dans certains cas particuliers, ils répondent à la dernière question. En particulier, dans ces cas, on établit une formule reliant la cohomologie de ces espaces à l'induction parabolique de celle de certains espaces de Rapoport-Zink de dimension inférieure (et dans les cas plus favorables basiques). Cette formule a été précédemment conjecturée par Harris dans [11] (Conjecture 5.2, p. 420). 


\section{Introduction}

1.1. - In [31] Rapoport and Zink introduce some local analogues of (PEL) type Shimura varieties, in the category of rigid analytic spaces over a $p$-adic local field. (PEL) type Shimura varieties arise as moduli spaces of abelian varieties with additional structures, namely endomorphisms, polarizations and level structures. Similarly, the spaces constructed by Rapoport and Zink are moduli spaces of Barsotti-Tate groups with the analogous additional structures.

A conjecture of Langlands predicts that some cases of correspondences between automorphic representations and global Galois representations are realized inside the cohomology of Shimura varieties. Analogously, a conjecture of Kottwitz (which is heuristically compatible with the conjecture of Langlands) predicts that some cases of correspondences between admissible representations of $p$-adic groups and local Galois representations are realized in the cohomology of the spaces constructed by Rapoport and Zink. (For linear groups over a local field, both the local case of Langlands' conjecture and Kottwitz's conjecture have been proved, respectively in [12], and [8].)

In the global case, the construction of Shimura associates to certain algebraic groups $G / \mathbb{Q}$ (together with a conjugacy class of homomorphisms $h: \mathbb{S} \rightarrow G_{\mathbb{R}}$ ) a projective system of varieties defined over a number field $E$ (called the reflex field), whose cohomology groups (regarded as an inductive limit) are naturally representations of the product of two groups: the points of $G$ over the finite adeles of $\mathbb{Q}, G\left(\mathbb{A}_{f}\right)$, and the absolute Galois group of $E$. Conjecturally, these groups realize the correspondence between automorphic representations of $G$ and representations of the global Weil group of $E$. In the local case, the construction of Rapoport and Zink depends not only on a choice of an algebraic group $G / \mathbb{Q}_{p}$ (together with a conjugacy class of cocharacters $\mu: \mathbb{G}_{m} \rightarrow G$ ) but also on a further datum $b$ associated with $(G, \mu)$. Moreover, the cohomology groups of the Rapoport-Zink spaces (again regarded as an inductive limit) are naturally representations of the product of three groups: the $p$-adic group $G\left(\mathbb{Q}_{p}\right)$, the Weil group of the local reflex field, $W_{E}$, and a second $p$-adic group $\mathrm{J}_{b}$ depending on the new data, also of the form $J_{b}=J_{b}\left(\mathbb{Q}_{p}\right)$, for an algebraic group $J_{b} / \mathbb{Q}_{p}$.

The presence of a third group raises important new questions. If we maintain our focus on the algebraic group $G$, a first goal is understanding the role played by the data $(b, \mu)$, and in particular, whether all admissible pairs would be relevant in a proof of the existence of the local Langlands correspondence for the group $G$ via the study of the cohomology of the Rapoport-Zink spaces. This question amounts to investigate for which pairs $(b, \mu)$ the cohomology of the associated Rapoport-Zink spaces contains supercuspidal representations of $G\left(\mathbb{Q}_{p}\right)$.

More completely, one would like to understand the role played by the group $J_{b}$. Indeed, Kottwitz's conjecture predicts that the cohomology of the Rapoport-Zink spaces not only realizes (some cases of) the local Langlands correspondence for the group $G$, but also for the group $J_{b}$, for each $b$. Furthermore, the obvious compatibility between the two correspondences (due to the fact that they are realized inside the same cohomology groups) would be an example of Langlands' functoriality principle (for each $b$ the group $J_{b}$ is an inner form of a Levi subgroup of $G$ ). Equivalently, the cohomology groups of the Rapoport-Zink spaces conjecturally also realize a generalized Jacquet-Langlands correspondence for $J_{b}$ and $G$.

$4^{\text {e }}$ SÉRIE - TOME $41-2008-\mathrm{N}^{\mathrm{o}} 5$ 
Kottwitz's predictions provide a conjectural answer to the first question we raised. In fact, they imply that supercuspidal representations of $G\left(\mathbb{Q}_{p}\right)$ should appear only in the cohomology groups of the Rapoport-Zink spaces associated with pairs $(b, \mu)$ for which $J_{b}$ is an inner form of $G$ (such pairs are called basic). More precisely, they predict that, for any admissible pair $b$, the admissible representations of $G\left(\mathbb{Q}_{p}\right)$ which arise in the cohomology of the associated Rapoport-Zink spaces are parabolically induced from the Levi subgroup of $G\left(\mathbb{Q}_{p}\right)$ which is an inner form of $J_{b}$.

Extending Kottwitz's predictions, in [11] (Conjecture 5.2, p. 420) Harris conjectured that the $l$-adic cohomology of non-basic Rapoport-Zink spaces is equal (in the appropriate Grothendieck group) to the non-normalized parabolic induction of the $l$-adic cohomology of the corresponding basic spaces, for a specified choice of the associated parabolic subgroup. Such a reduction of the computation of the $l$-adic cohomology of the Rapoport-Zink spaces associated with a group $G$ to that of the basic cases for $G$ and its Levi subgroups can be viewed as mirroring the results describing the admissible representations of $G\left(\mathbb{Q}_{p}\right)$ in terms of the supercuspidal representations of $G\left(\mathbb{Q}_{p}\right)$ and of its Levi subgroups. In the case of $G=G L_{n}$ and $\mu=(0, \ldots, 0,1)$ (i.e. in the case of Drinfeld's modular varieties) Harris' conjecture was already known, due to the work of Boyer ([4]), and played an important role in the proof of the existence of the local Langlands' correspondence for $G L_{n}$ in [12], and consequently also in [8].

1.2. - Let $G=\operatorname{Res}_{F_{0} / \mathbb{Q}_{p}}\left(G_{0}\right)$, for $F_{0} / \mathbb{Q}_{p}$ an unramified finite extension and $G_{0}=G L_{n}$ or $G S p_{2 n}$, for some integer $n \geq 1$. The goal of this paper is to investigate the above conjectures for the admissible pairs $(b, \mu)$ associated with such a group $G / \mathbb{Q}_{p}$. In particular, we will establish some instances of a variant of the conjecture of Harris.

Let us fix $\mu$ and consider the set $B(G, \mu)$ of all possible $b$ 's such that the pair $(b, \mu)$ is admissible. This is defined as a subset of the set of $\sigma$-conjugacy classes in $G(K)$, for $K$ the maximal unramified extension of $\mathbb{Q}_{p}$ and $\sigma$ its Frobenius automorphism. Its definition is originally due to Kottwitz who in [17] and [19] studied the set $B(G)$ of all $\sigma$-conjugacy classes in $G(K)$, for $G$ any connected reductive group over $\mathbb{Q}_{p}$. This set classifies isomorphism classes of $F$-isocrystals with $G$-structures over $k$, for $k$ the residue field of the ring of integers of $K$. Indeed, each element $b \in G(K)$ defines an exact faithful tensor functor $N_{b}$ from the category of $p$-adic representations of $G$ to that of $F$-isocrystals over $k$, via $N_{b}(W, \rho)=$ $\left(W \otimes K, \rho(b)\left(\operatorname{id}_{W} \otimes \sigma\right)\right)$. It follows from the definition that any such functor $N$ is defined by a unique $b \in G(K)$, and that if $b, b^{\prime}$ are $\sigma$-conjugate in $G(K)$ then the corresponding functors $N_{b}, N_{b^{\prime}}$ are isomorphic. For each $b \in G(K)$, the group $J_{b}$ is by definition the group of automorphisms of the $F$-isocrystal with $G$-structures $N_{b}$. (Thus if $b, b^{\prime}$ are $\sigma$-conjugate in $G(K)$ then the associated groups $J_{b}, J_{b^{\prime}}$ are isomorphic.) In the cases we are interested in, any such functor $N$ is uniquely determined by its value on the natural representation of $G$. Therefore, in these cases, an $F$-isocrystal with $G$-structures (defined as an exact faithful tensor functor) is simply an $F$-isocrystal (in the classical sense) together with additional structures, namely given endomorphisms and/or a non-degenerate alternating pairing.

We return to these cases, i.e. to $G$ as at the beginning of the section, and denote by $V$ the natural representation of $G$. To each $b \in G(K)$, we associate the Newton polygon $\nu_{b}$ of the corresponding $F$-isocrystal with $G$-structures over $k, N_{b}(V)$. Then the set $B(G, \mu)$ is realized 
as a subset of the set of convex polygons with integral break-points and the same end-points (Newton polygons), all lying above a fixed convex polygon, also with integral break-points and the same end-points, associated with $\mu$ (the Hodge polygon). It follows that there is a natural partial order on the set $B(G, \mu)$ : for any two elements $b, b^{\prime}$ in $B(G, \mu)$, we say $b \geq b^{\prime}$ if all points of $\nu_{b}$ lie below or on $\nu_{b^{\prime}}$. Under this partial ordering (which is called the Bruhat ordering), $B(G, \mu)$ has unique maximal element, which is called $\mu$-ordinary (and the corresponding admissible pair ordinary), and a unique minimal element corresponding to the basic pair. (A group-theoretic description of the Newton map for $G$ any connected reductive group over $\mathbb{Q}_{p}$ is discussed by Rapoport and Richartz in [30].)

Let $b_{0}$ be the $\mu$-ordinary element in $B(G, \mu)$. The following definition is justified by the results in [24] (we report on them in section 6). We say that an element $b \in B(G, \mu)$ (or the corresponding admissible pair $(b, \mu)))$ is of (HN) type if there is a break-point $x$ of $\nu_{b}$ which lies on $\nu_{b_{0}}$ and the two polygons coincide up to $x$ or from $x$ on. We call such a break-point $x$ of $\nu_{b}$ also of (HN) type. We remark that when $G$ is symplectic the polygon $\nu_{b}$ is symmetric (for any $b$ ). Thus, for each break-point $x$ of $\nu_{b}$ there is an associated symmetrical break-point $\hat{x}$. Furthermore, it is an easy observation that $x$ is of $(\mathrm{HN})$ type if and only if $\hat{x}$ is of $(\mathrm{HN})$ type. To each $b$ of (HN) type we attach a Levi subgroup $M_{b}$ of $G$ as follows. Every break-point $x=\left(x_{1}, x_{2}\right) \in \mathbb{Z}^{2}$ of $\nu_{b}$ defines a decomposition of the $F$-isocrystal $N_{b}(V)=V^{1} \oplus V^{2}$, for $V^{1}, V^{2}$ the two sub- $F$-isocrystals of $N_{b}(V)$ characterized by the properties that $V^{1}$ has Newton polygon $\nu_{1}$ consisting of the first $x_{1}$ slopes of $\nu_{b}$ and $V^{2}$ has Newton polygon $\nu_{2}$ consisting of the remaining slopes of $\nu_{b}$. Then, to any subset $S$ of the set of break-points of $\nu_{b}$ we associate the unique common refinement of the decompositions of $N_{b}(V)$ corresponding to each $x \in S$. It follows from the definition that any such decomposition is coarser than or equal to the slope decomposition (i.e. the decomposition of $N_{b}(V)$ into isoclinic factors), which in these notations is the decomposition associated with the set of all break-points of $\nu_{b}$. In particular, it follows from the analogous statements for the slope decomposition that any such decomposition of $V$ is $F_{0}$-linear, for $F_{0} / \mathbb{Q}_{p}$ the field extension as at the beginning of the section, and in the case when $G$ is symplectic compatible with the symplectic pairing on $V$ if the set $S$ is symmetrical, i.e. satisfying the condition $x \in S$ if and only if $\hat{x} \in S$. For each $b \in B(G, \mu)$ of $(\mathrm{HN})$ type we define $M_{b}$ to be the stabilizer in $G$ of the decomposition of $V$ into $p$-adic vector subspaces, which underlies the decomposition of the $F$-isocrystal $N_{b}(V)$ associated with the set of all break-points of $\nu_{b}$ of (HN) type. It follows from the definition that $M_{b}$ is a Levi subgroup of $G$. Finally, we also write $L_{b}$ for the stabilizer in $G$ of the decomposition of $V$ underlying the slope decomposition of $N_{b}(V)$. Then, $L_{b}$ is also a Levi subgroup of $G$ and it is an inner form of $J_{b}$ (see [17], Section 5.2, p. 215; [31], Corollary 1.14, p. 11). It follows from the definition that for each $b$ of (HN) type $M_{b} \supseteq L_{b}$, and $M_{b}=L_{b}$ if all the break-points of $\nu_{b}$ are of (HN) type (i.e. when all but possibly one of the sides of $\nu_{b}$ lie on the $\mu$-ordinary polygon $\nu_{b_{0}}$ ).

In this paper, we prove that if the admissible pair $(b, \mu)$ is of $(\mathrm{HN})$ type then the $l$-adic cohomology groups of the associated Rapoport-Zink spaces contain no supercuspidal representations. More precisely, we prove, for pairs of $(\mathrm{HN})$ type, a variant of Harris' conjecture which shows that, as representations of $G\left(\mathbb{Q}_{p}\right)$, these cohomology groups are parabolically induced from $M_{b}\left(\mathbb{Q}_{p}\right)$ to $G\left(\mathbb{Q}_{p}\right)$. In the special cases when $M_{b}=L_{b}$, e.g. for $b=b_{0}$, we prove Harris' conjecture. 
1.3. - The strategy in this paper can be described as follows. Let us fix a conjugacy class $\mu$ of cocharacters of $G$. For any non-basic element $b \in B(G, \mu) \subset B(G)$, there exists a proper Levi subgroup $M$ of $G$ such that $b$ arises as the image of an element of $B(M)$ (which with abuse of notations we still denote by $b$ ) under the natural map $B(M) \rightarrow B(G)$. It is clear from the definition that such a Levi subgroup $M$ of $G$ is not unique. On the other hand, it follows from the definition of $L_{b}$ that each $b \in B(G)$ arises as the image of a basic element of $B\left(L_{b}\right)$, and thus as the image of an element in $B(M)$, for any $M \supset L_{b}$. Furthermore, for each $b \in B(G, \mu)$, there exists a unique conjugacy class of cocharacters $w_{b} \mu$ of $L_{b}$ (which is the image of $\mu$ under conjugation by the element $w_{b} \in W$, the Weyl group of $G$ ) such that $b \in B\left(L_{b}\right)$ is $w_{b} \mu$-admissible and thus $b \in B\left(L_{b}, w_{b} \mu\right)$ the unique basic element (see [17], Proposition 6.2, p. 219; [11], Proposition 4.1, p. 415). It follows that, for any $M \supset$ $L_{b}, b \in B\left(M, w_{b} \mu\right)$ (here, with a second abuse of notation we still write $w_{b} \mu$ for the class of cocharacters of $M$ induced by that of $L_{b}$ via the inclusion $\left.L_{b} \subset M\right)$. In particular, any element $b \in B(G, \mu)$ of (HN) type arises as the image of an element in $B\left(M_{b}, w_{b} \mu\right)$.

We state a variant of Harris' conjecture. It predicts that, given a connected reductive group $G$ and a conjugacy class of its cocharacters $\mu$ in the class considered in [31], for any $\mu$-admissible element $b \in B(G, \mu)$ and any Levi subgroup $M$ of $G$ for which the pair $\left(b, w_{b} \mu\right)$ as described above is admissible, the $l$-adic cohomology of the Rapoport-Zink spaces associated with the data $(G, b, \mu)$ is equal to the non-normalized parabolic induction, from $M\left(\mathbb{Q}_{p}\right)$ to $G\left(\mathbb{Q}_{p}\right)$ of that of the Rapoport-Zink spaces corresponding to the data $\left(M, b, w_{b} \mu\right)$. Then, Harris' conjecture (as stated in [11]) is, for any group $G$ and admissible pair $(b, \mu)$, the case $M=L_{b}$ of this variant. On the other hand, if we assume Harris' conjecture to hold not only for $G$ but also for its Levi subgroups, then the above variant follows.

In this paper we prove this variant of Harris' conjecture under the assumptions that the pair $(b, \mu)$ is of $(\mathrm{HN})$ type and $M \supset M_{b}$, for a group $G$ as in section 1.2. We remark that, for a given pair $(b, \mu)$, the general case of $M \supset M_{b}$ follows from the special case $M=M_{b}$, as we compare the result for the group $G$ with those corresponding to its Levi subgroups containing $M_{b}$.

Finally, we point out that although the conclusive results obtained in this paper only apply to admissible pairs of ( $\mathrm{HN})$ type (and the associated Levi subgroups), most of the work is carried out for any admissible pair (and any Levi subgroup satisfying the above condition).

1.4. - We outline in more detail the structure of this paper.

Let $G / \mathbb{Q}_{p}$ be an unramified algebraic group as in section 1.2. For a given admissible pair $(b, \mu)$, we write $L=L_{b}$ and $J=J_{b}$. Assume $b$ is non-basic and let $M$ be a proper Levi subgroup of $G$ containing $L$. Then $b \in B\left(M, w_{b} \mu\right)$ and to the pair $(b, \mu)$ we associate two different systems of Rapoport-Zink spaces, namely the one attached to the data $(G, b, \mu)$, which we denote by $\mathcal{M}^{\infty}$, and the one attached to $\left(M, b, w_{b} \mu\right), \mathcal{P}^{\infty}$ (which is the corresponding basic one for $M=L$ ). It is a simple observation that there exists a morphism (or more precisely a compatible system of morphisms) $\Theta: \mathcal{P}^{\infty} \rightarrow \mathcal{M}^{\infty}$, which is equivariant for the action of the groups $M\left(\mathbb{Q}_{p}\right)$ and $J\left(\mathbb{Q}_{p}\right)$, as well as for the descent data over the local reflex field $E$ (for simplicity, we refer to the descent data as the action of $W_{E}$ ) (Sections 2 and 3).

To each Levi subgroup $M$ as above we associate a parabolic subgroup $P=P_{M}$ of $G$ as follows. Let $V=\oplus_{i} V^{i}$ be the decomposition of $V$ associated with $M$. Since $M \supset L$ 
this decomposition underlies a decomposition of $F$-isocrystals. We order the $V^{i}$ s according to the increasing order of the slopes of $\nu_{b}$ and consider the filtration $V_{\bullet}$ of $V$ defined by $V_{j}=\oplus_{0 \leq i \leq j} V^{i}$. We define $P=P_{M}$ to be the stabilizer in $G$ of this filtration. Then, $P$ is a parabolic subgroup of $G$ with $M$ as a Levi subgroup.

Our idea is to introduce a third system of rigid analytic spaces, which we denote by $\mathcal{F}^{\infty}$, associated with the data $\left(P, b, w_{b} \mu\right)$, for $P=P_{M} . \mathcal{F}^{\infty}$ is also defined as a system of moduli spaces of Barsotti-Tate groups with additional structures (among which the datum of a filtration), and similarly to the case of the Rapoport-Zink spaces is endowed with an action of $P\left(\mathbb{Q}_{p}\right), J\left(\mathbb{Q}_{p}\right)$ and $W_{E}$ (Section 4).

The new system $\mathcal{F}^{\infty}$ enjoys the following properties:

- there exists a morphism $j: \mathcal{F}^{\infty} \rightarrow \mathcal{M}^{\infty}$, which is equivariant for the actions of $P\left(\mathbb{Q}_{p}\right) \subset G\left(\mathbb{Q}_{p}\right), J\left(\mathbb{Q}_{p}\right)$ and $W_{E} ;$

- the morphism $\Theta: \mathcal{P}^{\infty} \rightarrow \mathcal{M}^{\infty}$ factors through $j$, and the corresponding morphism $\mathcal{P}^{\infty} \rightarrow \mathcal{F}^{\infty}$ (which we still denote as $\Theta$ ) is equivariant for the actions of $M\left(\mathbb{Q}_{p}\right) \subset$ $P\left(\mathbb{Q}_{p}\right), J\left(\mathbb{Q}_{p}\right)$ and $W_{E}$

- there exists a morphism $\Xi: \mathcal{F}^{\infty} \rightarrow \mathcal{P}^{\infty}$, which is equivariant for the actions of $P\left(\mathbb{Q}_{p}\right)$, $J\left(\mathbb{Q}_{p}\right)$ and $W_{E}$ (where the action of $P\left(\mathbb{Q}_{p}\right)$ on $\mathcal{P}^{\infty}$ is defined via the epimorphism $P \rightarrow M)$, and which satisfies the condition $\Xi \circ \Theta=1_{\mathcal{P} \infty}$.

We achieve our goal, by comparing the cohomology of these three systems, as representations of the above groups.

Let $l$ be a prime number, $l \neq p$. For each tower $Y=\mathcal{P}^{\infty}, \mathcal{F}^{\infty}, \mathcal{M}^{\infty}$, we consider its $l$-adic étale cohomology groups $H^{i}(Y)$ (more precisely, we consider the direct limit of the étale cohomology groups with compact support and coefficients in $\mathbb{Q}_{l}\left(D_{Y}\right)$, for $\left.D_{Y}=\operatorname{dim} Y\right)$. The groups $H^{i}(Y)$ are naturally $l$-adic representations of $Q_{Y} \times W_{E}$ and $J\left(\mathbb{Q}_{p}\right)$ (for $Q_{Y}=M\left(\mathbb{Q}_{p}\right), P\left(\mathbb{Q}_{p}\right), G\left(\mathbb{Q}_{p}\right)$ respectively). These are not a priori admissible. On the other hand, for any $l$-adic admissible representation $\rho$ of $J\left(\mathbb{Q}_{p}\right)$, the following holds:

- the groups $\operatorname{Ext}_{J\left(\mathbb{Q}_{p}\right)}^{j}\left(H^{i}(Y), \rho\right)$ are admissible representations of $Q_{Y} \times W_{E}$,

- they vanish for almost all $i, j \in \mathbb{Z}_{\geq 0}$.

Thus, to each representation $\rho$ as above we associate the virtual representation of $Q_{Y} \times W_{E}$

$$
H^{\bullet}(Y)_{\rho}=\sum_{i, j}(-1)^{i+j} \operatorname{Ext}_{J\left(\mathbb{Q}_{p}\right)}^{j}\left(H^{i}(Y), \rho\right) .
$$

The main results proved in this paper are the following (Sections 7, 8 and 9).

THEOREM 1.1. - The map on cohomology $\left(\Xi^{*}\right)^{\vee}$ is an isomorphism. In particular, for any $l$-adic admissible representation $\rho$ of $J\left(\mathbb{Q}_{p}\right)$, we have an equality of virtual representations of $P\left(\mathbb{Q}_{p}\right) \times W_{E}:$

$$
H^{\bullet}\left(\mathcal{F}^{\infty}\right)_{\rho}=H^{\bullet}\left(\mathcal{P}^{\infty}\right)_{\rho}
$$

Theorem 1.2. - Let $\mathcal{M}$ (resp. $\mathcal{F}$ ) be a space in the tower $\mathcal{M}^{\infty}$ (resp. $\left.\mathcal{F}^{\infty}\right)$ corresponding to a choice of a maximal compact open subgroup $U_{0}$ of $G\left(\mathbb{Q}_{p}\right)$ (resp. to $U_{0} \cap P\left(\mathbb{Q}_{p}\right) \subset P\left(\mathbb{Q}_{p}\right)$ ). Then, the morphism $j$ maps $\mathcal{F}$ to $\mathcal{M}$, and we denote by $\mathcal{M}^{\infty} \times_{\mathcal{M}} \mathcal{F}$ the pullback of the tower $\mathcal{M}^{\infty}$ over $\mathcal{F}$ via $j$.

$4^{\mathrm{e}}$ SÉRIE - TOME $41-2008-\mathrm{N}^{\mathrm{o}} 5$ 
For any l-adic admissible representation $\rho$ of $J\left(\mathbb{Q}_{p}\right)$, we have an equality of virtual representations of $G\left(\mathbb{Q}_{p}\right) \times W_{E}$ :

$$
H^{\bullet}\left(\mathcal{M}^{\infty} \times_{\mathcal{M}} \mathcal{F}\right)_{\rho}=\operatorname{Ind}_{P\left(\mathbb{Q}_{p}\right)}^{G\left(\mathbb{Q}_{p}\right)}\left(H^{\bullet}\left(\mathcal{F}^{\infty}\right)_{\rho}\right) .
$$

Theorem 1.3. - Let $\mathcal{M}$ be as above. Then, there exists a closed rigid analytic subspace $\mathcal{X} \subset \mathcal{M}$, which is invariant under the actions of $J\left(\mathbb{Q}_{p}\right)$ and $W_{E}$, and satisfies the following conditions.

Let $\mathcal{X}^{\infty}=\mathcal{M}^{\infty} \times_{\mathcal{M}} \mathcal{X}$ denote the pullback of the tower $\mathcal{M}^{\infty}$ over $\mathcal{X}$. Then, for any l-adic admissible representation $\rho$ of $J\left(\mathbb{Q}_{p}\right)$ :

1. there is an equality of virtual representations of $G\left(\mathbb{Q}_{p}\right) \times W_{E}$ :

$$
H^{\bullet}\left(\mathcal{X}^{\infty}\right)_{\rho}=H^{\bullet}\left(\mathcal{M}^{\infty} \times \mathcal{M} \mathcal{F}\right)_{\rho}
$$

2. there is an equality of virtual representations of $W_{E}$ :

$$
H^{\bullet}\left(\mathcal{X}^{\infty}\right)_{\rho}^{U_{0}}=H^{\bullet}\left(\mathcal{M}^{\infty}\right)_{\rho}^{U_{0}} .
$$

These results hold for any admissible pair $(b, \mu)$ and any Levi subgroup $M$ of $G$ which contains $L_{b}$.

Finally, under the assumption that the admissible pair $(b, \mu)$ is of $(\mathrm{HN})$ type and for the associated Levi subgroup $M \supseteq M_{b}$, we prove one last result (Section 10).

Theorem 1.4. - Assume $(b, \mu)$ is of $(H N)$ type, and $M \supseteq M_{b}$. Then, $\mathcal{X}=\mathcal{M}$.

We observe that the equality $\mathcal{X}=\mathcal{M}$ holds in particular in the case of $G=G L_{n}, \mu=$ $(0, \ldots, 0,1)$ and any $\mu$-admissible $b$, as in the work of Boyer.

We also remark that for a general admissible pair $(b, \mu)$ (i.e. neither basic nor of $(\mathrm{HN})$ type), $\mathcal{X}$ is a proper subspace of $\mathcal{M}$.

Under the assumptions of theorem 1.4, our results imply the corresponding instances of the variant of Harris' conjecture we discussed.

Corollary 1.5. - Assume $(b, \mu)$ is of (HN) type, and $M \supseteq M_{b}$. Then, for any l-adic admissible representation $\rho$ of $J\left(\mathbb{Q}_{p}\right)$, we have an equality of virtual representations of $G\left(\mathbb{Q}_{p}\right) \times W_{E}:$

$$
H^{\bullet}\left(\mathcal{M}^{\infty}\right)_{\rho}=\operatorname{Ind}_{P\left(\mathbb{Q}_{p}\right)}^{G\left(\mathbb{Q}_{p}\right)}\left(H^{\bullet}\left(\mathcal{P}^{\infty}\right)_{\rho}\right)
$$

Since the tower $\mathcal{F}^{\infty}$ consists of moduli spaces of filtered Barsotti-Tate groups with additional structures, many questions regarding its geometry (and how it compares to the geometry of $\mathcal{P}^{\infty}$ and $\mathcal{M}^{\infty}$ ) are translated into questions regarding the notion of a filtration on a Barsotti-Tate group. More precisely, we focus on the notion of a slope filtration, which is the filtration corresponding to our choice of the parabolic subgroup $P_{L}$, for any $\mu$-admissible $b$, and of a Hodge-Newton filtration, which corresponds to the parabolic subgroup $P_{b}=P_{M_{b}}$, for $b$ of (HN) type. In particular, we investigate some of their properties, such as existence, splitting and behavior with respect to the additional structures on the Barsotti-Tate groups, both in characteristic $p$ and characteristic 0 (Sections 5 and 6). 
1.5. - We conclude the paper with an application of these results to the theory of Shimura varieties. More precisely, given a Shimura datum of (PEL) type $(G, h)$ over $\mathbb{Q}$, which is unramified at $p$, there exists a natural stratification (the Newton polygon stratification) of the reduction modulo $p$ of the corresponding Shimura varieties, by locally closed reduced subschemes which are stable under the action of $G\left(\mathbb{A}_{f}\right)$ (for $\mathbb{A}_{f}$ the finite adeles of $\mathbb{Q}$ ). This stratification, which we denote by $\left\{\overline{\mathcal{S}}_{b}^{\infty}\right\}_{b}$, is indexed by the set $B\left(G_{\mathbb{Q}_{p}}, \mu_{h}\right)$ (where $G_{\mathbb{Q}_{p}}$ denotes the $p$-adic component of the algebraic group $G / \mathbb{Q}$ and $\mu_{h}$ the conjugacy class of a cocharacter of $G_{\mathbb{Q}_{p}}$ corresponding to $h$ ).

For each $b \in B\left(G_{\mathbb{Q}_{p}}, \mu_{h}\right)$, the cohomology groups of the corresponding Newton polygon strata, with compact supports and coefficients in the vanishing cycles of $\mathbb{Q}_{l}$, $H_{c}^{p}\left(\overline{\mathcal{S}}_{b}^{\infty}, R^{q} \Psi_{\eta}\left(\mathbb{Q}_{l}\right)\right)$ for all $p, q \geq 0$, are closely related to the $l$-adic cohomology of the associated systems of Rapoport-Zink spaces (see [22],[23]). In particular, corollary 1.5 implies the following result. (Section 11).

Corollary 1.6. - Let $E$ denote the reflex field of the Shimura datum $(G, h), E_{v}$ its completion at a prime $v \mid p$ and $W_{E_{v}}$ its local Weil group. Let $b \in B\left(G_{\mathbb{Q}_{p}}, \mu_{h}\right)$. Assume b is of $(H N)$ type.

Then, the virtual representation of $G\left(\mathbb{A}_{f}\right) \times W_{E_{v}}$

$$
H_{c}^{\bullet}\left(\overline{\mathcal{S}}_{b}^{\infty}, R^{\bullet} \Psi_{\eta}\left(\mathbb{Q}_{l}\right)\right)=\sum_{p, q \geq 0}(-1)^{p+q} H_{c}^{p}\left(\overline{\mathcal{S}}_{b}^{\infty}, R^{q} \Psi_{\eta}\left(\mathbb{Q}_{l}\right)\right)
$$

contains no supercuspidal representations of $G\left(\mathbb{Q}_{p}\right)$.

\section{Acknowledgements}

I am very grateful to J. de Jong, M. Harris and R. Taylor for their interest in my work and stimulating support, and to the Institut des Hautes Études Scientifiques for its hospitality. In preparing this paper I also benefited a lot from many mathematical discussions with L. Fargues, B. Moonen, F. Oort, K. Ribet and E. Viehmann. It is a pleasure to thank them all. Finally, I thank the referee for a careful reading of the paper and for a number of useful suggestions. This work was partially supported by the National Science Foundation (NSF) Grant DMS-0701310.

\section{Rapoport-Zink spaces}

In this section, we recall the definition of the moduli spaces of Barsotti-Tate groups constructed by Rapoport and Zink in [31]. These are distinguished in two types. The first type classifies Barsotti-Tate groups with given endomorphisms and level structures, the second one classifies Barsotti-Tate groups with given endomorphisms, polarizations and level structures. Following [31], we refer to them respectively as (EL) and (PEL).

Let $p$ be an odd prime number. We introduce the data defining the moduli problems.

$4^{\text {e }}$ SÉRIE - TOME $41-2008-\mathrm{N}^{\mathrm{o}} 5$ 
2.1. - Let $B$ be a finite-dimensional semi-simple algebra over $\mathbb{Q}_{p}$ and $V$ a finite-dimensional $B$-module. We denote by $F$ the center of $B$. Then $F$ is a finite product of finite field extensions of $\mathbb{Q}_{p}$. In this paper, we only deal with the case of $B$ (and thus $F$ ) unramified, i.e. $B$ splits over an unramified extension of $\mathbb{Q}_{p}$ (containing all factors of $F$ ).

In the (PEL) case, we further require the data of:

1. an involution $*$ on $B$,

2. a non degenerate alternating $*$-hermitian $\mathbb{Q}_{p}$-bilinear form (, ) on $V$,

$$
(b v, w)=\left(v, b^{*} w\right), \quad \text { for all } v, w \in V, b \in B .
$$

To the above data we associate an algebraic group $G / \mathbb{Q}_{p}$. In the (EL) case, $G=G L_{B}(V)$. In the (PEL) case, we define $G=G U_{B}(V,()$,$) , the group of the B$-linear automorphisms of $V$ preserving the pairing (, ) up to a constant, i.e. for any $\mathbb{Q}_{p}$-algebra $R$ we set

$G(R)=\left\{g \in G L_{B}(V \otimes R) \mid\right.$ there exists $c(g) \in R^{\times}:(g v, g w)=c(g)(v, w)$ for all $\left.v, w \in V\right\}$.

2.2. - Let $k$ be an algebraic closure of $\mathbb{F}_{p}, W(k)$ its Witt vector ring, and $K_{0}=W(k) \otimes \mathbb{Q}_{p}$ the fraction field of $W(k)$. We write $\sigma$ for the Frobenius on $K_{0}$.

To any element $b \in G\left(K_{0}\right)$, we associate a structure of isocrystal on $V$, namely

$$
N(V)=N_{b}(V)=\left(V \otimes K_{0}, \Phi=b\left(1_{V} \otimes \sigma\right)\right) .
$$

The isocrystal is naturally endowed with an action of $B$ and in the (PEL) case with an alternating form

$$
\psi: N(V) \otimes N(V) \rightarrow K_{0}(n),
$$

where $K_{0}(n)$ denotes the isocrystal $\left(K_{0}, p^{n} \sigma\right)$, for $n=v l_{p}(c(b))$, which is defined as $\psi(v, w)=u^{-1} \cdot(v, w), v, w \in N(V)$, for $u$ a unit in $W(k)$ such that $c(b)=p^{n} u \sigma(u)^{-1}$. By definition $\psi$ is only determined up to a $\mathbb{Q}_{p}^{\times}$-multiple.

The isocrystal $N_{b}(V)$, together with its additional structures, depends up to isomorphism only on the $\sigma$-conjugacy class of $b$. In the following, we write $B(G)$ for the set $G\left(K_{0}\right) / \sim_{\sigma}$. Thus, given the data $(B, V)$, the isocrystal $N_{b}(V)$ depends on a choice of an element $b \in$ $B(G)$. To any $b \in B(G)$ we also associate an algebraic group $J=J_{b}$ over $\mathbb{Q}_{p}$, with points in a $\mathbb{Q}_{p}$-algebra $R$

$$
J_{b}(R)=\left\{g \in G\left(R \otimes_{\mathbb{Q}_{p}} K_{0}\right) \mid g^{\sigma}=b^{-1} g b\right\} .
$$

In addition to the above data, we fix a conjugacy class of cocharacters $\mu: \mathbb{G}_{m} \rightarrow G$. To a cocharacter $\mu$, defined over a finite extension $K$ of $K_{0}$, we associate the filtration $V_{i}$ of $V_{K}$, given by the weight spaces with respect to $\mu$. A pair $(b, \mu)$ is said to be admissible if such is the associated filter isocrystal.

In the following, we always assume:

1. the pair $(b, \mu)$ is admissible;

2. the isocrystal $N_{b}(V)$ has slopes in the interval $[0,1]$;

3. the weight decomposition of $N_{b}(V)$ with respect to $\mu$ contains only weights 0 and 1 : $V_{K}=V_{0} \oplus V_{1}$;

4. in the PEL case, $\operatorname{val}_{p}(c(b))=1$. 
2.3. - Equivalently, let $\mathcal{O}_{B}$ be a maximal order of $B$, and in the PEL case assume that $\mathcal{O}_{B}$ is fixed by the involution $*$ and that there exists a self-dual $\mathcal{O}_{B}$-lattice $\Lambda \subset V$. (This is always possible for $B$ unramified.)

Then, the filtered isocrystal $N(V)$ is the filtered isocrystal of a Barsotti-Tate group $\hat{\Sigma}$, defined over the ring of integers $\mathcal{O}_{K}$ of $K$, where $\hat{\Sigma}$ is endowed with an action of $\mathcal{O}_{B}$ and in the (PEL) case with a polarization $\ell$ satisfying the conditions $\ell \circ b^{*}=b^{\vee} \circ \ell$, for all $b \in \mathcal{O}_{B}$ (where $b^{\vee}$ denotes the isogeny dual to $b$ ). (By a polarization of a Barsotti-Tate group $\hat{\Sigma}$ we mean an antisymmetric isomorphism $\ell: \hat{\Sigma} \rightarrow \hat{\Sigma}^{\vee}$.)

The filtered isocrystal $N(V)$ depends up to isomorphisms on the isogeny class of the reduction $\Sigma=\hat{\Sigma}_{k}$ of $\hat{\Sigma}$ modulo the maximal ideal of $\mathcal{O}_{K}$; and the group $J\left(\mathbb{Q}_{p}\right)$ is the group of the self-quasi-isogenies of $\Sigma$. (By an isogeny between Barsotti-Tate groups with additional structure we mean an isogeny of the underlying Barsotti-Tate groups which commutes with the action of $\mathcal{O}_{B}$ and in the (PEL) case preserves the polarization $\ell$ up to a $\mathbb{Q}_{p}^{\times}$-multiple.)

2.4. - We remark that associated with the choice of a maximal order $\mathcal{O}_{B}$ of $B$ and of a $\mathcal{O}_{B^{-}}$ lattice $\Lambda$ in $V$ as above, one can define

$$
\begin{gathered}
U_{0}=\left\{g \in G\left(\mathbb{Q}_{p}\right) \mid g \Lambda=\Lambda\right\}, \\
U_{m}=\left\{g \in U_{0} \mid g_{\mid \Lambda} \equiv 1_{\Lambda} \quad \bmod p^{m} \Lambda\right\}, \quad m \in \mathbb{Z}_{>0},
\end{gathered}
$$

where $U_{0}$ is a maximal open compact subgroup of $G\left(\mathbb{Q}_{p}\right)$, and the $U_{m}$ 's, as $m$ varies, form a basis of neighborhoods of the identity in $G\left(\mathbb{Q}_{p}\right)$.

2.5. - We now recall the definition of the moduli spaces of Barsotti-Tate groups associated with the data $(B, V, b, \mu)$ as above. For simplicity, we introduce the moduli problems in terms of a choice of a Barsotti-Tate group $\Sigma / k$ with additional structures of isocrystal $N_{b}(V)$ (via covariant Dieudonné theory). It is easy to see that the corresponding moduli spaces depend up to isomorphism only on the isogeny class of $\Sigma / k$.

Let $E$ be the field of definition of the conjugacy class of $\mu$, and $E_{0}$ the maximal unramified extension of $E$ with residue field $k$. (When the moduli data is unramified, $E$ is an unramified extension of $\mathbb{Q}_{p}$ and we can identify $E_{0}=K_{0}$.) We write $\mathcal{O}_{E}$ (resp. $\mathcal{O}_{E_{0}}$ ) for the ring of integers of $E$ (resp. $E_{0}$ ), and Nilp $\mathcal{O}_{E_{0}}$ for the category of $\mathcal{O}_{E_{0}}$-schemes $S$ where $p$ is locally nilpotent. For any $S \in \operatorname{Nilp}_{\mathcal{O}_{E_{0}}}$ we denote $\bar{S}$ the closed subscheme of $S$ defined by $p \mathcal{O}_{S}$. We consider $\bar{S}$ as a $k$-scheme via the natural map $k \rightarrow \mathcal{O}_{E_{0}} / p \mathcal{O}_{E_{0}}$.

The moduli spaces associated by Rapoport and Zink to the above data are rigid analytic spaces over $E_{0}$, indexed by the open compact subgroups $U$ of $G\left(\mathbb{Q}_{p}\right)$. (For each space we call the corresponding subgroup $U$ its level.) Without loss of generality, we may assume $U \subseteq U_{0}$.

Definition 2.1 ([31], Chapters 3 and 5). - Let $\mathcal{M}$ be the contravariant functor on Nilp $\mathcal{O}_{E_{0}}$ which to a scheme $S$ associates the set of isomorphisms classes of pairs $(H, \beta)$, where $H$ is a Barsotti-Tate groups over $S$, endowed with an action of $\mathcal{O}_{B}$ and in the (PEL) case with a polarization, and $\beta: \Sigma \times_{k} \bar{S} \rightarrow H_{\bar{S}}$ is a quasi-isogeny (of Barsotti-Tate groups with additional structure), satisfying the condition

$$
\operatorname{det}_{\mathcal{O}_{S}}(b, \operatorname{Lie}(H))=\operatorname{det}_{K}\left(b, V_{0}\right) \text {, for all } b \in \mathcal{O}_{B} .
$$

$4^{\text {e }}$ SÉRIE - TOME $41-2008-\mathrm{N}^{\mathrm{o}} 5$ 
Then, the functor $\mathcal{M}$ is represented by a formal scheme, which is formally locally of finite type over $\operatorname{Spf}\left(\mathcal{O}_{E_{0}}\right)$.

Let $\mathcal{M}^{\text {rig }}$ denote the rigid analytic fiber of $\mathcal{M}$ over $E_{0}$, and let $\mathcal{T} / \mathcal{M}^{\text {rig }}$ be the local system defined by the $p$-adic Tate module of the universal Barsotti-Tate group on $\mathcal{M}$. For any $U \subseteq U_{0}$, we define $\mathcal{M}^{U}$ to be the finite étale covering of $\mathcal{M}^{\text {rig }}$ parameterizing the classes modulo $U$ of $\mathcal{O}_{B}$-linear trivializations of $\mathcal{T}$ by $\Lambda$. In the (PEL) case, we further require that the trivializations preserve the alternating pairings up to a $\mathbb{Q}_{p}^{\times}$-multiple.

We remark that $\mathcal{M}^{\text {rig }}=\mathcal{M}^{U_{0}}$. The following facts are a direct consequence of the above definitions.

1. The formal scheme $\mathcal{M}$ (and therefore also the spaces $\mathcal{M}^{U}$, for all $U$ ) depends up to isomorphism only on the isogeny class of $\Sigma / k$.

2. There is a non-effective descent datum on $\mathcal{M}$ over $\mathcal{O}_{E}$ (and thus compatibly also on each $\mathcal{M}^{U}$ over $E$ ). More precisely, let $k(E) \subset k$ be the residue field of $E, \tau$ the Frobenius automorphism of $k$ relative to $k(E)$ (i.e. $\tau(x)=x^{q}$ for $q=\# k(E)$ ), and $F_{E}: \Sigma \rightarrow \tau^{*} \Sigma$ the relative Frobenius morphism of Barsotti-Tate groups. Then, the descent datum is defined as

$$
(H, \beta) \mapsto\left(H, \beta \circ F_{E}^{-1}\right) .
$$

3. The group $J\left(\mathbb{Q}_{p}\right)=J_{b}\left(\mathbb{Q}_{p}\right)$ of the self-quasi-isogenies of $\Sigma$ which are compatible with the additional structures acts on $\mathcal{M}$ (and thus also on the $\mathcal{M}^{U}$ ). For any $\gamma \in J\left(\mathbb{Q}_{p}\right)$ we define its action as

$$
(H, \beta) \mapsto\left(H, \beta \circ \gamma^{-1}\right) .
$$

4. As the level $U$ varies, the rigid analytic spaces $\mathcal{M}^{U}$ form a projective system, naturally endowed with an action of $G\left(\mathbb{Q}_{p}\right)$.

Finally, we recall that when the moduli data is unramified the formal scheme $\mathcal{M}$ is known to be formally smooth and therefore the spaces $\mathcal{M}^{U}$ are smooth rigid analytic spaces. (This result is due to Kottwitz, in [18], Section 5.)

2.6. In the following, we study the $l$-adic étale cohomology groups of the Rapoport-Zink spaces as representations of $G\left(\mathbb{Q}_{p}\right) \times W_{E}$ and $J\left(\mathbb{Q}_{p}\right)$. More precisely, let $l$ be a prime number, $l \neq p$, and $\hat{\bar{E}}_{0}$ the completion of an algebraic closure of $E_{0}$. For any level $U$, we consider the cohomology groups

$$
H^{i}\left(\mathcal{M}^{U}\right)=H_{c}^{i}\left(\mathcal{M}^{U} \times_{E_{0}} \hat{\overline{E_{0}}}, \mathbb{Q}_{l}\left(D_{\mathcal{M}}\right)\right)=\underline{\lim } \mathcal{U} H_{c}^{i}\left(\mathcal{U} \times_{E_{0}} \hat{\bar{E}_{0}}, \mathbb{Q}_{l}\left(D_{\mathcal{M}}\right)\right)
$$

where $\mathcal{U}$ varies among the open subspaces of $\mathcal{M}^{U}$ of finite type, and $D_{\mathcal{M}}=\operatorname{dim} \mathcal{M}^{U}(i \in \mathbb{N})$. On them, there is a natural action of the groups $J\left(\mathbb{Q}_{p}\right)$ and $W_{E}$, induced by the action of $J\left(\mathbb{Q}_{p}\right)$ and the descent data over $E$ on the space $\mathcal{M}^{U}$.

As the level $U$ varies, these cohomology groups form a direct limit which is natural endowed with an action of $G\left(\mathbb{Q}_{p}\right)$ induced by the action of $G\left(\mathbb{Q}_{p}\right)$ on the tower $\mathcal{M}^{\infty}$. Moreover, the following result holds.

TheOREM 2.2 ([22], section 8.2). - Let $\rho$ be an admissible l-adic representation of $J\left(\mathbb{Q}_{p}\right)$.

1. The groups $H^{i, j}\left(\mathcal{M}^{\infty}\right)_{\rho}=\lim _{U} \operatorname{Ext}_{J\left(\mathbb{Q}_{p}\right)}^{j}\left(H^{i}\left(\mathcal{M}^{U}\right), \rho\right)$ vanish for almost all $i, j \geq 0$.

2. There is a natural action of $G\left(\mathbb{Q}_{p}\right) \times W_{E}$ on the $H^{i, j}\left(\mathcal{M}^{\infty}\right)_{\rho}$. 


\section{The representations $H^{i, j}\left(\mathcal{M}^{\infty}\right)_{\rho}$ are admissible.}

Given any admissible $l$-adic representation $\rho$ of $J\left(\mathbb{Q}_{p}\right)$, we define a virtual representation of $G\left(\mathbb{Q}_{p}\right) \times W_{E}$

$$
H^{\bullet}\left(\mathcal{M}^{\infty}\right)_{\rho}=\sum_{i, j \geq 0}(-1)^{i+j} H^{i, j}\left(\mathcal{M}^{\infty}\right)_{\rho}
$$

\section{Basic Rapoport-Zink spaces}

We fix some moduli data $(B, V)$, and a maximal order $\mathcal{O}_{B}$ of $B$ with a $\mathcal{O}_{B}$-lattice $\Lambda$ of $V$ as in the previous section.

For a minuscule class of cocharacters $\mu$, we denote by $B(G, \mu)$ the subset of $B(G)$ consisting of all $\sigma$-conjugacy classes $b$ such that the pair $(b, \mu)$ is admissible. Under our hypothesis, the set $B(G, \mu)$ is in bijection with the set of the Newton polygons of the associated isocrystals. Moreover, for all $b \in B(G, \mu)$ the associated isocrystals share the same end-points and the same Hodge polygon, which are uniquely determined by $\mu$. The set $B(G, \mu)$ can therefore be endowed with the partial ordering "lies below" on the Newton polygons (which is called the Bruhat ordering). There is a unique minimal element for such ordering, called basic, and a unique maximal one, called $\mu$-ordinary. It follows from results of Grothendieck ([10], p. 149; [15], Theorem 2.3.1, p. 143) and Oort ([28], Theorem 2.1, p. 422) that the Bruhat ordering is equivalent to the ordering under specialization on the corresponding isogenies classes of Barsotti-Tate groups, e.g. in the Zariski topology, being $\mu$-ordinary is an open condition while being basic is a closed condition. Also, an equivalent condition for $b \in B(G, \mu)$ to be basic is that the algebraic group $J_{b}$ of automorphisms of the associated isocrystal $N_{b}(V)$ is an inner form of $G$ ([17], Section 5.2, p. 215). The set $B(G, \mu)$ (resp. $B(G)$ ) can be described uniquely in terms of the basic elements in $B(L)$ as $L$ varies among the Levi subgroups of $G$. More precisely, to any admissible pair $(b, \mu)$ of $G$, one can associate a choice of a Levi subgroup $L=L_{b}$ of $G$ and a class of cocharacter $w_{b} \mu$ of $L$ such that $b \in B\left(L, w_{b} \mu\right)$ and is basic (see [17], Proposition 6.2, p. 219; [11], Proposition 4.1, p. 415). In the following, we recall the details of this construction in the cases of our interest.

3.1. - Given an admissible pair $(b, \mu)$ of $G$, let $N=N_{b}(V)$ be the filtered isocrystal associated with the pair $(b, \mu), V_{1} \subset V_{K}$ its filtration, for $K$ a finite extension of $K_{0}$ (notations as in 2.2). We write $\Phi$ for the $\sigma$-linear Frobenius on $N$. Then, the choice of $b \in B(G)$ uniquely determines the Newton polygon of $N$, and viceversa the Newton polygon of $N$ uniquely determines $b$. On the other hand, while $\mu$ uniquely determines the Hodge polygon of $N$, the viceversa does not hold in general. We remark that under our hypothesis a simple refinement of the datum of the Hodge polygon does. More precisely, let $F$ be the unramified finite extension of $\mathbb{Q}_{p}$ which is the center of $B, r=\left[F: \mathbb{Q}_{p}\right]$, and define $I=\operatorname{Hom}_{\mathbb{Q}_{p}}\left(F, K_{0}\right)$. For convenience, we fix $j \in I$ and identify the set $I$ with $\mathbb{Z} / r \mathbb{Z}$, via the map $s \mapsto \sigma^{s} \circ j$. Then, we have a natural decomposition $V=\oplus_{i \in I} V^{(i)}$, where $V^{(i)}=\{v \in V \mid \lambda \cdot v=i(\lambda) v$ for all $\lambda \in F\}$. This is not a decomposition of the $\sigma$-isocrystal $N$ overlying $V$, since it is not stable under the Frobenius endomorphism $\Phi$. In fact, for each $i \in I, \Phi$ maps the space $V^{(i)}$ to $V^{(i+1)}$. On the other hand, it follows from the above observation that this decomposition is stable under $\Phi^{r}$, and thus it can be regarded as a decomposition of the $\sigma^{r}$-isocrystal $\left(N, \Phi^{r}\right)$. For each $i \in I$, 
we write $\left(N^{(i)}, \Phi^{r} \mid N^{(i)}\right)$, for the $\sigma^{r}$-isocrystal overlying $V^{(i)}$. It is a simple observation that the $N^{(i)}$ 's are all isogenous, and that their (common) Newton polygon uniquely determines the Newton polygon of $(N, \Phi)$. The analogous statement for the Hodge polygons does not hold in general. On the other hand, the decomposition $V=\oplus_{i} V^{(i)}$ gives rise to a decomposition of the Hodge polygon of $N$, and the datum of the Hodge polygon together with this decomposition uniquely determines $\mu$ (see [31], Section 3.23, Part b, pp. 83-84; [32], Section 2.3, pp. 588-591).

3.2. - For an admissible pair $(b, \mu)$ of $G$, let us now consider the slope decomposition of the associated isocrystal $N(V)=\oplus_{s} N\left(W^{s}\right)$, with $N\left(W^{s}\right)$ isoclinic of slope $\lambda_{s}$. We number the slopes of $N(V)$ in decreasing order, $1 \geq \lambda_{1}>\cdots>\lambda_{r} \geq 0$. It is an easy consequence of the uniqueness of the slope decomposition that it is preserved under the additional structures. More precisely, the action of $B$ preserves the subspaces $N\left(W^{s}\right)$, for all $s$, and in the (PEL) case, the alternating form on $N(V)$ induces a perfect pairing on $N\left(W^{s}\right) \otimes N\left(W^{u}\right)$, for all $s, u$ such that $s+u=r+1$.

We write $V=\oplus_{s} W^{s}$ for the corresponding decomposition of $V$ into sub- $B$-modules. We define $L=L_{b}$ to be the stabilizer of this decomposition in $G, \bar{b}$ the element in $B(L)$ corresponding to the Newton polygons of the isocrystals $N\left(W^{s}\right)$, and $w_{b} \mu$ the class of cocharacters of $L$ corresponding to their refined Hodge polygons (in the sense of section 3.1). It follows from the definition that $L$ is a Levi subgroup of $G$, and $\bar{b}$ a $w_{b} \mu$-admissible element of $B(L)$, which is sent to $b \in B(G)$ under the natural map $B(L) \rightarrow B(G)$. Moreover, $L$ is an inner form of $J_{b}$ (see [31], Corollary 1.14, p. 11), thus $\bar{b} \in B\left(L, w_{b} \mu\right)$ is basic. In the following, with abuse of notations, we write $\bar{b}=b$.

Let $M$ be any Levi subgroup of $G$ containing $L=L_{b}$. Then $M$ is the Levi subgroup of $G$ associated with a (in the (PEL) case symplectic) $B$-linear decomposition of $V, V=\oplus_{i} V^{i}$, which is coarser than or equal to the slope decomposition $V=\oplus_{s} W^{s}$. (In the (PEL) case, we say that a $B$-linear decomposition $V=\oplus_{i} V^{i}$ is symplectic if the alternating pairing on $V$ induces perfect pairings between $V^{i}$ and $V^{t+1-i}$, for all $i=1, \ldots, t$.) Viceversa, for any partition $\left(n_{1}, \ldots, n_{t}\right)$ of $r$ (in the (PEL) case satisfying the conditions $n_{i}=n_{t+1-i}$, for all $i$ ), let $M$ be the Levi subgroup associated to the decomposition $V=\oplus_{i} V^{i}$ defined as

$$
V^{i}=\oplus_{n_{i-1}+1}^{n_{i}} W^{s}, \quad \text { for all } i=1, \ldots, t \text { and } n_{0}=0 .
$$

Then, $M$ contains $L$. It follows from the definition that the image of $b$ under the natural map $B(L) \rightarrow B(M)$ is the element in $B(M)$ corresponding to the Newton polygons of the isocrystals $N\left(V^{i}\right)=\oplus_{n_{i-1}+1}^{n_{i}} N\left(W^{s}\right)$, and that the class of cocharacters of $M$ induced by $w_{b} \mu$ is the one associated with their refined Hodge polygons.

In the following, we write $V_{0}^{i}$ for the weight 0 subspace of $V_{K}^{i}$, for each $i=1, \ldots, t$.

3.3. - Given the data $(B, V, b, \mu)$, we choose a decomposition $V=\oplus_{i} V^{i}$ as above (we call such a decomposition compatible with the data $(b, \mu)$ ), and write $M$ for the associated Levi subgroup of $G$.

To these data we can associate a second tower of moduli spaces of Barsotti-Tate groups, which we denote by $\mathcal{P}^{\infty}=\mathcal{P}_{M}^{\infty}=\left\{\mathcal{P}^{U}=\mathcal{P}_{M}^{U}\right\}_{U \subset M\left(\mathbb{Q}_{p}\right)}$, which is the tower of RapoportZink spaces associated with the data $\left(B, \oplus_{i} V^{i}, M, b, w_{b} \mu\right)$. In this paper, we investigate how, 
for any given admissible pair $(b, \mu)$, the geometry and the cohomology of $\mathcal{M}^{\infty}$ can be explained in terms of the geometry and cohomology of the associated $\mathcal{P}_{M}^{\infty}$ 's.

3.4. - As in section 2.6, by the cohomology of $\mathcal{P}^{\infty}$ we mean the following data. Let $l$ be a prime number, $l \neq p$. We consider the cohomology groups $(i \in \mathbb{N})$

$$
H^{i}\left(\mathcal{P}^{U}\right)=H_{c}^{i}\left(\mathcal{P}^{U} \times_{E_{0}} \hat{\bar{E}_{0}}, \mathbb{Q}_{l}\left(D_{\mathcal{P}}\right)\right)=\underline{\lim } \mathcal{V} H_{c}^{i}\left(\mathcal{V} \times_{E_{0}} \hat{\bar{E}_{0}}, \mathbb{Q}_{l}\left(D_{\mathcal{P}}\right)\right)
$$

where $\mathcal{V}$ varies among the open subspaces of $\mathcal{P}^{U}$ of finite type, and $D_{\mathcal{P}}=\operatorname{dim} \mathcal{P}^{U}$, endowed with the actions of $J\left(\mathbb{Q}_{p}\right)$ and $W_{E}$ induced by the action of $J\left(\mathbb{Q}_{p}\right)$ and the descent data over $E$ on the space $\mathcal{P}^{U}$. As the level $U$ varies, these cohomology groups form a projective limit which is natural endowed with an action of $M\left(\mathbb{Q}_{p}\right)$ induced by the action of $M\left(\mathbb{Q}_{p}\right)$ on $\mathcal{P}^{\infty}$. Then, to any admissible $l$-adic representation $\rho$ of $J\left(\mathbb{Q}_{p}\right)$, we associate a virtual representation of $M\left(\mathbb{Q}_{p}\right) \times W_{E}$

$$
H^{\bullet}\left(\mathcal{P}^{\infty}\right)_{\rho}=\sum_{i, j \geq 0}(-1)^{i+j} H^{i, j}\left(\mathcal{P}^{\infty}\right)_{\rho} .
$$

In the following, we also consider the above groups as representations of $P\left(\mathbb{Q}_{p}\right) \times W_{E}$, for $P$ a parabolic subgroup with $M$ as a Levi factor, by letting the unipotent radical of $P\left(\mathbb{Q}_{p}\right)$ act trivially on them.

3.5. - We describe the construction of the spaces $\mathcal{P}^{U}$ more explicitly. We choose the lattice $\Lambda$ of the form $\Lambda=\oplus_{i} \Lambda^{i}$, for $\Lambda^{i} \subset V^{i}$ a $\mathcal{O}_{B}$-lattices, $i=1, \ldots, t$. In the (PEL) case we further assume that the restriction of the pairing $($,$) to \Lambda$ induces perfect pairings between the sublattices $\Lambda^{i}$ and $\Lambda^{j}$, for $i, j$ satisfying $i+j=t+1$. Then, the associated Barsotti-Tate group $\Sigma / k$ is of the form $\Sigma=\oplus_{i} \Sigma^{i}$, where $\Sigma^{i}$ is a Barsotti-Tate group with isocrystal $V^{i}$, for each $i=1, \ldots, t$. It follows from the definition that the action of $\mathcal{O}_{B}$ on $\Sigma$ preserves the above decomposition, and in the (PEL) case, that the polarization $\ell$ of $\Sigma$ induces isomorphisms $\ell^{i}: \Sigma^{i} \rightarrow\left(\Sigma^{\vee}\right)^{i}=\left(\Sigma^{j}\right)^{\vee}$, satisfying the conditions $\left(\ell^{\vee}\right)^{i}=c \cdot \ell^{j}, c \in \mathbb{Z}_{p}^{\times}$, for all $i, j$ such that $i+j=t+1$. For $\Sigma$ of the form $\Sigma=\oplus_{s} \mathbb{X}^{s}$, where for each $s$ the Barsotti-Tate group $\mathbb{X}^{s}$ is isoclinic of slope $\lambda_{s}, 1 \geq \lambda_{1}>\cdots>\lambda_{r} \geq 0$, we have $\Sigma^{i}=\oplus_{n_{i-1}+1}^{n_{i}} \mathbb{X}^{s}$ for all $i$ (notations as in 3.2).

Let $U_{0} \subset M\left(\mathbb{Q}_{p}\right)$ be the maximal compact subgroup associated with our choice of an integral lattice $\Lambda=\oplus_{i} \Lambda^{i} \subset V=\oplus_{i} V^{i}$. For simplicity, we treat the (EL) and (PEL) cases separately. (We maintain the notations introduced in section 2.5.)

3.6. - In the (EL) case, let $\mathcal{P}$ be the formal scheme representing the contravariant functor that to a scheme $S \in \operatorname{Nilp}_{\mathcal{O}_{E_{0}}}$ associates the set of isomorphism classes of $t$ pairs $\left\{\left(H^{i}, \beta^{i}\right)\right\}_{i=1, \ldots, t}$ where

1. $H^{i}$ are Barsotti-Tate groups over $S$ with an action of $\mathcal{O}_{B}$;

2. $\beta^{i}: \Sigma_{\bar{S}}^{i} \rightarrow H_{\bar{S}}^{i}$ are quasi-isogeny, commuting with the action of $\mathcal{O}_{B}$;

satisfying the conditions, for $i=1, \ldots, t$,

$$
\operatorname{det}_{\mathcal{O}_{S}}\left(b, \operatorname{Lie}\left(H^{i}\right)\right)=\operatorname{det}_{K}\left(b, V_{0}^{i}\right) \text { for all } b \in \mathcal{O}_{B} .
$$

Let $\mathcal{T}=\oplus_{i} \mathcal{T}^{i} / \mathcal{P}^{\text {rig }}$ be the local system defined by the $p$-adic Tate module of the universal Barsotti-Tate group on $\mathcal{P}$. Then, for any $U \subseteq U_{0}$, the space $\mathcal{P}^{U}$ is the finite étale covering over $\mathcal{P}^{\text {rig }}$ parameterizing the classes modulo $U$ of $\mathcal{O}_{B}$-linear trivializations of $\mathcal{T}=\oplus_{i} \mathcal{T}^{i}$ by 
$\Lambda=\oplus_{i} \Lambda^{i}$, which are compatible with the decompositions. (With these notations, $\mathcal{P}^{U_{0}}=$ $\mathcal{P}^{\text {rig }}$.)

Equivalently, we can describe the system $\mathcal{P}^{\infty}$ as follows. Let $G^{i}$ denote the algebraic group associated to the pair $\left(B, V^{i}\right)$ (i.e. the group of $B$-linear automorphisms of $\left.V^{i}\right)$. Then, $M=$ $G^{1} \times \cdots \times G^{t}$. For all $i=1, \ldots, t$, we write $\left(b^{i}, \mu^{i}\right)$ for the restriction of $\left(b, w_{b} \mu\right)$ to the factor $G^{i}$, and denote by $\mathcal{M}_{i}^{\infty}$ the tower of Rapoport-Zink spaces associated with the data $\left(B, V^{i}, b^{i}, \mu^{i}\right)$.

Then, it follows from the above description that, for any level $U$ of the form $U=U_{1} \times \cdots \times U_{t}$, we can identify

$$
\mathcal{P}^{U}=\mathcal{M}_{1}^{U_{1}} \times \cdots \times \mathcal{M}_{t}^{U_{t}} .
$$

Therefore, by the Künneth formula, for any $l$-adic admissible representation $\rho=\otimes_{i} \rho_{i}$ of $J\left(\mathbb{Q}_{p}\right)=J_{b}\left(\mathbb{Q}_{p}\right)=J_{b^{1}}\left(\mathbb{Q}_{p}\right) \times \cdots \times J_{b^{t}}\left(\mathbb{Q}_{p}\right)$, there is an equality of virtual representations of $M\left(\mathbb{Q}_{p}\right) \times W_{E}$

$$
H^{\bullet}\left(\mathcal{P}^{\infty}\right)_{\rho}=H^{\bullet}\left(\mathcal{M}_{1}^{\infty}\right)_{\rho_{1}} \otimes \cdots \otimes H^{\bullet}\left(\mathcal{M}_{t}^{\infty}\right)_{\rho_{t}} .
$$

3.7. - In the (PEL) case, let $\mathcal{P}$ be the formal scheme representing the contravariant functor that to a scheme $S \in \mathrm{Nilp}_{\mathcal{O}_{E_{0}}}$ associates the set of isomorphism classes of $t$ triples $\left\{\left(H^{i}, \ell_{H^{i}}, \beta^{i}\right)\right\}_{i=1, \ldots, t}$ where

1. $H^{i}$ are Barsotti-Tate groups over $S$ with an action of $\mathcal{O}_{B}$;

2. $\ell_{H^{i}}: H^{i} \rightarrow\left(H^{j}\right)^{\vee}, j=t+1-i$, are isomorphisms and $\ell_{H^{i}}^{\vee}=-\ell_{H^{j}}$;

3. $\beta^{i}: \Sigma_{\bar{S}}^{i} \rightarrow H_{\bar{S}}^{i}$ are quasi-isogenies, commuting with the action of $\mathcal{O}_{B}$;

satisfying the conditions

1. $\operatorname{det}_{\mathcal{O}_{S}}\left(b, \operatorname{Lie}\left(H^{i}\right)\right)=\operatorname{det}_{K}\left(b, V_{0}^{i}\right)$, for all $b \in \mathcal{O}_{B}$ and $i=1, \ldots, t$;

2. there exists $c \in \mathbb{Q}_{p}^{\times}$such that $\ell_{H^{i}}=c \cdot\left(\beta^{j \vee}\right)^{-1} \circ \ell^{i} \circ\left(\beta^{i}\right)^{-1}$ for all $i, j$ with $i+j=t+1$.

Let $\mathcal{T}=\oplus_{i} \mathcal{T}^{i} / \mathcal{P}^{\text {rig }}$ be the local system defined by the $p$-adic Tate module of the universal Barsotti-Tate group over $\mathcal{P}$. Then, for any $U \subseteq U_{0}$, the space $\mathcal{P}^{U}$ is the finite étale covering over $\mathcal{P}^{\text {rig }}$ parameterizing the classes modulo $U$ of $\mathcal{O}_{B}$-linear trivializations of $\mathcal{T}=\oplus_{i} \mathcal{T}^{i}$ by $\Lambda=\oplus_{i} \Lambda^{i}$, which are compatible with the decompositions and preserve the pairing up to a constant. (Again, with these notations, $\mathcal{P}^{U_{0}}=\mathcal{P}^{\text {rig }}$.)

It follows from the above description that in the (PEL) case the first $\left[\frac{t+1}{2}\right]$-triples completely determine the remaining ones. More precisely, we have a description analogous to the one in the (EL) case. We distinguish the cases of $t$ even and $t$ odd.

For $t$ odd, let $i=1, \ldots,(t+1) / 2$, and write $G^{i}$ for the algebraic group associated to the pair $\left(B, V^{i}\right)$ (i.e. the group of $B$-linear automorphisms of $V^{i}$ if $i \neq(t+1) / 2$, and the group of $B$-linear automorphisms of $V^{i}$ preserving the pairing up to scalar multiple if $i=$ $(t+1) / 2)$. Then, $M=G^{1} \times \cdots \times G^{(t+1) / 2}$. For all $i=1, \ldots,(t+1) / 2$, we write $\left(b^{i}, \mu^{i}\right)$ for the restriction of $\left(b, w_{b} \mu\right)$ to the factor $G^{i}$, and denote by $\mathcal{M}_{i}^{\infty}$ the tower of RapoportZink spaces associated with the data $\left(B, V^{i}, b^{i}, \mu^{i}\right)$. (These spaces are of (EL) type for $i \neq$ $(t+1) / 2$ and of (PEL) type for $i=(t+1) / 2$.)

Then, it follows from the above description that, for any level $U$ of the form $U=U_{1} \times \cdots \times U_{(t+1) / 2} \subset M\left(\mathbb{Q}_{p}\right)$, we can identify

$$
\mathcal{P}^{U}=\mathcal{M}_{1}^{U_{1}} \times \cdots \times \mathcal{M}_{(t+1) / 2}^{U_{(t+1) / 2}} .
$$


Therefore, using the Künneth formula, for any $l$-adic admissible representation $\rho=\otimes_{i} \rho_{i}$ of $J\left(\mathbb{Q}_{p}\right)=J\left(\mathbb{Q}_{p}\right)_{b}=J_{b^{1}}\left(\mathbb{Q}_{p}\right) \times \cdots \times J_{b^{(t+1) / 2}}\left(\mathbb{Q}_{p}\right)$, there is an equality of virtual representations of $M\left(\mathbb{Q}_{p}\right) \times W_{E}$

$$
H^{\bullet}\left(\mathcal{P}^{\infty}\right)_{\rho}=H^{\bullet}\left(\mathcal{M}_{1}^{\infty}\right)_{\rho_{1}} \otimes \cdots \otimes H^{\bullet}\left(\mathcal{M}_{(t+1) / 2}^{\infty}\right)_{\rho_{(t+1) / 2}} .
$$

For $t$ even, let $i=1, \ldots, t / 2$, and $G^{i}$ as above. Then $M=\mathbb{G}_{m} \times G^{1} \times \cdots \times G^{t / 2}$, and it follows from the above description that, for any level $U$ of the form $U=K \times U_{1} \times \cdots \times U_{t / 2} \subset$ $M\left(\mathbb{Q}_{p}\right)$, we can identify

$$
\mathcal{P}^{U}=\coprod_{\mathbb{Q}_{p}^{\times} / K} \mathcal{M}_{1}^{U_{1}} \times \cdots \times \mathcal{M}_{t / 2}^{U_{t / 2}} .
$$

Therefore, using the Künneth formula, for any $l$-adic admissible representation $\rho=\chi \otimes$ $\left(\otimes_{i} \rho_{i}\right)$ of $J\left(\mathbb{Q}_{p}\right)=J_{b}\left(\mathbb{Q}_{p}\right)=\mathbb{Q}_{p}^{\times} \times\left(J_{b^{1}}\left(\mathbb{Q}_{p}\right) \times \cdots \times J_{b^{t / 2}}\left(\mathbb{Q}_{p}\right)\right)$, there is an equality of virtual representations of $M\left(\mathbb{Q}_{p}\right) \times W_{E}$

$$
H^{\bullet}\left(\mathcal{P}^{\infty}\right)_{\rho}=\left(\mathbb{Q}_{l}\right)_{\chi} \otimes H^{\bullet}\left(\mathcal{M}_{1}^{\infty}\right)_{\rho_{1}} \otimes \cdots \otimes H^{\bullet}\left(\mathcal{M}_{t / 2}^{\infty}\right)_{\rho_{t / 2}}
$$

3.8. - To the inclusion of the Levi subgroup $M$ into $G$ corresponds a system of equivariant morphisms

$$
\Theta^{\infty}: \mathcal{P}^{\infty} \rightarrow \mathcal{M}^{\infty}
$$

We first define a morphism of formal schemes over $\mathcal{O}_{E_{0}}, \Theta: \mathcal{P} \rightarrow \mathcal{M}$ as

$$
\begin{gathered}
(E L): \quad\left\{\left(H^{i}, \beta^{i}\right)\right\}_{i} \mapsto\left(H=\oplus_{i} H^{i}, \beta=\oplus_{i} \beta^{i}\right), \\
(P E L): \quad\left\{\left(H^{i}, \ell_{H^{i}}, \beta^{i}\right)\right\}_{i} \mapsto\left(H=\oplus_{i} H^{i}, \ell_{H}=\oplus_{i} \ell_{H^{i}}, \beta=\oplus_{i} \beta^{i}\right),
\end{gathered}
$$

Indeed, for any $\left\{\left(H^{i}, \beta^{i}\right)\right\}_{i} \in \mathcal{P}(S), S \in \operatorname{Nilp}_{\mathcal{O}_{E_{0}}}$, we may endow the Barsotti-Tate group $H=\oplus_{i} H^{i}$ with an action of $\mathcal{O}_{B}$ defined from the action on the $H^{i}$. The quasi-isogeny $\beta$ is thus $\mathcal{O}_{B}$-equivariant. Furthermore, in the (PEL) case, it is easy to check that the isomorphism $\ell_{H}: H \rightarrow H^{\vee}$ is antisymmetric (thus a polarization), and that it is compatible with the polarization $\ell$ on $\Sigma$, via the quasi-isogeny $\beta$. Finally, we have $\operatorname{Lie}(H)=\oplus_{i} \operatorname{Lie}\left(H^{i}\right)$, $V_{0}=\oplus_{i} V_{0}^{i}$ and

$$
\operatorname{det}_{\mathcal{O}_{S}}(b, \operatorname{Lie}(H))=\prod_{i} \operatorname{det}_{\mathcal{O}_{S}}\left(b, \operatorname{Lie}\left(H^{i}\right)\right)=\prod_{i} \operatorname{det}_{K}\left(b, V_{0}^{i}\right)=\operatorname{det}_{K}\left(b, V_{0}\right) .
$$

For any $U \subset G\left(\mathbb{Q}_{p}\right)$, we write $\mathcal{P}^{U}=\mathcal{P}^{U \cap M\left(\mathbb{Q}_{p}\right)}$. Then, by the universal properties of the covers $\mathcal{M}^{U} \rightarrow \mathcal{M}^{U_{0}}$, we see that the morphism $\Theta$ canonically lifts to a system of morphisms of rigid analytic spaces $\Theta^{U}: \mathcal{P}^{U} \rightarrow \mathcal{M}^{U}$, which are compatible with the natural projections, as the level $U$ varies. Moreover, they commute with the descent data over $E$, with the action of $J\left(\mathbb{Q}_{p}\right)=J_{b}\left(\mathbb{Q}_{p}\right)$, and with action of $M\left(\mathbb{Q}_{p}\right) \subset G\left(\mathbb{Q}_{p}\right)$ on the projective systems.

This system of morphisms is the starting point of our analysis.

\section{Moduli spaces of filtered Barsotti-Tate groups}

In this section, we introduce a third kind of moduli spaces of Barsotti-Tate groups associated with the data $(B, V, b, \mu)$. 
4.1. - Let $V=\oplus_{i} V^{i}$ be a decomposition of $V$ compatible with the choice of the data $(b, \mu)$ as in section 3.2. We define an increasing filtration $V_{\bullet}$ of $V$,

$$
0 \subset V_{1} \subset \cdots \subset V_{t}=V \text {, as } V_{j}=\oplus_{1 \leq i \leq j} V^{i} \text {. }
$$

The above filtration is the filtration on the isocrystal $N_{b}(V)$ associated with the following filtration of $\Sigma$,

$$
0 \subset \Sigma_{1} \subset \cdots \subset \Sigma_{t}=\Sigma, \text { for } \Sigma_{j}=\oplus_{1 \leq i \leq j} \Sigma^{i},
$$

where the components $\Sigma^{i}$ of $\Sigma$ are ordered according to the decreasing order of the slopes, i.e. for each $i=1, \ldots, t-1$ all the slopes of $\Sigma^{i}$ are strictly greater than those of $\Sigma^{i+1}$. Thus, in the case of the slope decomposition $V=\oplus_{i} W^{i}$, the associated filtration on $\Sigma$ is the natural slope filtration.

Let $P=P_{M}$ be the parabolic subgroup of $G$ associated with the above filtration of $V$. Then it follows from the definition that $M$ is a Levi factor of $P$.

To the data $\left(B, V_{\bullet}, P, b, w_{b} \mu\right)$ we associate a projective system of moduli spaces of Barsotti-Tate groups $\mathcal{F}^{\infty}=\left\{\mathcal{F}^{U}\right\}_{U \subset P\left(\mathbb{Q}_{p}\right)}$, indexed by the open compact subgroups of $P\left(\mathbb{Q}_{p}\right)$. Similarly to the previous two cases, the moduli spaces $\mathcal{F}^{U}$ we will define satisfy the following requirements:

1. for each level $U, \mathcal{F}^{U}$ is a rigid analytic space (locally of finite type) defined over $E_{0}$, and to each inclusion $U_{1} \subseteq U$ corresponds a transition map $\mathcal{F}^{U_{1}} \rightarrow \mathcal{F}^{U}$;

2. there is a non-effective descent datum on the $\mathcal{F}^{U}$ 's over $E \subset E_{0}$, which is compatible with the transition maps;

3. the group $J\left(\mathbb{Q}_{p}\right)=J_{b}\left(\mathbb{Q}_{p}\right)$ acts on the spaces $\mathcal{F}^{U}$ 's, compatibly with the transition maps;

4. as the level $U$ varies, the spaces $\mathcal{F}^{U}$ 's form a projective system (which we denote by $\mathcal{F}^{\infty}$ ) which is endowed with an action of $P\left(\mathbb{Q}_{p}\right)$.

4.2. - Let $\mathcal{O}_{B}$ be a maximal order of $B$, and $\Lambda=\oplus_{i} \Lambda^{i}$ an $\mathcal{O}_{B}$-lattice of $V$ as in section 3.5. We define an increasing filtration $\Lambda_{\bullet}$ of $\Lambda$ as

$$
0 \subset \Lambda_{1} \subset \cdots \subset \Lambda_{t}=\Lambda, \text { for } \Lambda_{j}=\oplus_{1 \leq i \leq j} \Lambda^{i} .
$$

Thus, for all $j=1, \ldots, t, \Lambda_{j}$ is a $\mathcal{O}_{B}$-lattice of $V_{j}$.

To the filtration $\Lambda_{\bullet}$, we associate a system of compact subgroups of $P\left(\mathbb{Q}_{p}\right)$,

$$
\begin{gathered}
U_{0}=\left\{g \in P\left(\mathbb{Q}_{p}\right) \mid g \Lambda_{j}=\Lambda_{j} \text { for all } j=1, \ldots, t\right\}, \\
U_{m}=\left\{g \in U_{0} \mid g_{\mid \Lambda} \equiv 1_{\Lambda} \quad \bmod p^{m} \Lambda\right\}, \text { for } m \in \mathbb{Z}_{>0} .
\end{gathered}
$$

Then $U_{0}$ is a maximal open compact subgroup of $P\left(\mathbb{Q}_{p}\right)$, and the $U_{m}$, as $m$ varies, form a basis of neighborhoods of the identity in $P\left(\mathbb{Q}_{p}\right)$. Thus, without loss of generality, we may always assume $U \subseteq U_{0}$ (and $U=U_{m}$, for some integer $m \geq 1$ ).

Definition 4.1. - Let $\mathcal{F}$ be the contravariant functor on $\operatorname{Nilp}_{\mathcal{O}_{E_{0}}}$ which to a scheme $S$ associates the set of isomorphisms classes of triples $\left(H, H_{\bullet}, \beta\right)$, where

1. $H$ is a Barsotti-Tate group over $S$, endowed with an action of $\mathcal{O}_{B}$ and in the (PEL) case with a polarization; 
2. $H_{\bullet}$ is an increasing filtration of $H$ by Barsotti-Tate groups over $S$, with Barsotti-Tate subquotients, which is preserved by the action of $\mathcal{O}_{B}$ and in the (PEL) case by the polarization;

3. $\beta: \Sigma_{\bar{S}} \rightarrow H_{\bar{S}}$ is a quasi-isogeny of Barsotti-Tate groups, compatible with the additional structures, i.e. with the action of $\mathcal{O}_{B}$ and in the (PEL) case the polarization, and with the filtration, i.e. $\beta\left(\Sigma_{j, \bar{S}}\right) \subseteq H_{j, \bar{S}}$, for any $j=1, \ldots, t$;

satisfying the conditions:

1. the restrictions of $\beta$ to the Barsotti-Tate subgroups defining the filtration

$$
\beta_{j}: \Sigma_{j, \bar{S}} \rightarrow H_{j, \bar{S}},
$$

are quasi-isogenies;

2. for all $j=1, \ldots, t$,

$$
\operatorname{det}_{\mathcal{O}_{S}}\left(b, \operatorname{Lie}\left(H_{j}\right)\right)=\operatorname{det}_{K}\left(b, V_{0, j}\right), \quad \text { for all } b \in \mathcal{O}_{b} .
$$

Let us assume for the moment that the functor $\mathcal{F}$ is represented by a formal scheme, which is formally locally of finite type over $\operatorname{Spf}\left(\mathcal{O}_{E_{0}}\right)$. Let $\mathcal{F}^{\text {rig }}$ denote the rigid analytic fiber of $\mathcal{F}$ over $E_{0}$, and $\mathcal{T} / \mathcal{F}^{\text {rig }}$ the local system defined by the $p$-adic Tate module of the universal Barsotti-Tate group over $\mathcal{F}$. Then, $\mathcal{T}$ has a natural filtration $\mathcal{T}_{\bullet}$ associated with the filtration of the universal Barsotti-Tate group.

Definition 4.2. - For any $U \subseteq U_{0}$, we define $\mathcal{F}^{U}$ to be the finite étale covering of $\mathcal{F}^{\text {rig }}$ parameterizing the classes modulo $U$ of $\mathcal{O}_{B}$-linear trivializations of $\mathcal{T}$ by $\Lambda$, mapping the filtration $\Lambda_{\bullet}$ of $\Lambda$ onto the filtration $\mathcal{T}$. of $\mathcal{T}$. In the (PEL) case we further require that the trivializations preserve the alternating pairings up to a $\mathbb{Q}_{p}^{\times}$-multiple.

Then, $\mathcal{F}^{U_{0}}=\mathcal{F}^{\text {rig }}$. It is an easy consequence of the definition that the spaces $\mathcal{F}^{U}$ satisfy the expected requirements. More precisely, let $F_{E}: \Sigma \rightarrow \tau^{*} \Sigma$ denote the relative Frobenius morphism of $\Sigma$. It is clear that the morphism $F_{E}$ maps the Barsotti-Tate subgroups $\Sigma_{j}$ onto $\tau^{*} \Sigma_{j}$, for all $j$. In fact the restriction $F_{E \mid \Sigma_{j}}: \Sigma_{j} \rightarrow \tau^{*} \Sigma_{j}$ is simply the relative Frobenius of the Barsotti-Tate groups $\Sigma_{j}$ (in particular, it is an isogeny). We define a non-effective descent datum on $\mathcal{F}$ over $\mathcal{O}_{E}$ (and thus compatibly on the $\mathcal{F}^{U}$ 's over $E$ ) as

$$
\left(H, H_{\bullet}, \beta\right) \mapsto\left(H, H_{\bullet}, \beta \circ F_{E}^{-1}\right) .
$$

Similarly, let $J\left(\mathbb{Q}_{p}\right)=J_{b}\left(\mathbb{Q}_{p}\right)$ be the group of the self-quasi-isogenies of $\Sigma$, which are compatible with its additional structures. Each $\gamma \in J\left(\mathbb{Q}_{p}\right)$ preserves the slope filtration of $\Sigma$, and thus the filtration $\Sigma_{\bullet}$. More over, for all $j=1, \ldots, t$ the restrictions $\gamma_{j}: \Sigma_{j} \rightarrow \Sigma_{j}$ are self-quasi-isogenies. We define an action of $J\left(\mathbb{Q}_{p}\right)$ on $\mathcal{F}$ (and thus also on the $\mathcal{F}^{U}$ 's) as

$$
\left(H, H_{\bullet}, \beta\right) \mapsto\left(H, H_{\bullet}, \beta \circ \gamma^{-1}\right) .
$$

As the level $U$ varies, the rigid analytic spaces $\mathcal{F}^{U}$ 's form a projective system $\mathcal{F}^{\infty}$, i.e. to each inclusion $V \subseteq U$ we associate the natural projection $\mathcal{F}^{V} \rightarrow \mathcal{F}^{U}$. The projective system $\mathcal{F}^{\infty}$ is naturally endowed with an action of $P\left(\mathbb{Q}_{p}\right)$, defined by composition on the right with the trivializations.

Finally, we remark that the spaces $\mathcal{F}^{U}$ 's only depend on the data $\left(B, V_{\bullet}, P, b, w_{b} \mu\right)$, i.e. on the isogeny class of $\Sigma / k$, and not on the choice of the Barsotti-Tate group $\Sigma / k$. 
Proposition 4.3. - The functor $\mathcal{F}$ is represented by a formal scheme over $\mathcal{O}_{E}$, formally locally of finite type.

Moreover, if we assume that the moduli data $\left(B, V_{\bullet}, P, b, \nu\right)$ is unramified, then the formal scheme $\mathcal{F}$ is formally smooth, and the rigid analytic spaces $\mathcal{F}^{U}$ 's smooth, for all $U \subset P\left(\mathbb{Q}_{p}\right)$.

Proof. - We prove that $\mathcal{F}$ is representable by proving that it is relatively representable over some related Rapoport-Zink spaces.

For any $j=1, \ldots, t$, we consider the Rapoport-Zink moduli space $\mathcal{M}_{j}$ associated with the Barsotti-Tate group $\Sigma_{j} / k$ (see definition 2.1). Then, there exists an obvious transformation of functors

$$
\mathcal{F} \rightarrow \prod_{j} \mathcal{M}_{j}
$$

which identify $\mathcal{F}$ with the subfunctor of $\prod_{j} \mathcal{M}_{j}$ where the Barsotti-Tate groups $H_{j}$ fit together as increasing filtration of $H_{t}$ and the quasi-isogenies $\beta_{j}$ agree with the restrictions of $\beta_{t}$, and in the (PEL) case the polarization of $H_{t}$ preserves the filtration. More precisely, for any $j=1, \ldots, t-1$, let $\iota_{j}: \Sigma_{j} \rightarrow \Sigma$ denote the natural inclusion and define

$$
\phi_{j}=\beta_{j+1} \circ \iota_{j} \circ \beta_{j}^{-1}: H_{j} \rightarrow H_{j+1} .
$$

Then, the $\phi_{j}$ are $\mathcal{O}_{B}$-linear quasi-morphisms and $\mathcal{F}$ is the subfunctor of $\prod_{j} \mathcal{M}_{j}$ where the $\phi_{j}$ are injective morphisms, which in the (PEL) case are compatible with the polarization on $H_{t}$. It follows from Proposition 2.9 and Lemma 2.10 (p. 52, in [31]) that the condition, that a quasi-morphism of Barsotti-Tate groups over a scheme $S$ is a morphism, is represented by a closed subscheme. Moreover, the condition that a morphism is injective is represented by an open subscheme, and in the (PEL) case the condition that the filtration is compatible with the polarization by a closed subscheme. Thus, $\mathcal{F}$ is relatively representable and therefore represented by a formal scheme over $\mathcal{O}_{E_{0}}$, which is formally locally of finite type since such are the $\mathcal{M}_{j}$ 's.

For $(B, V)$ unramified, the fact that the formal scheme $\mathcal{F}$ is formally smooth can be proved using the deformation theory of Grothendieck-Messing, similarly to the case of the moduli spaces considered by Rapoport and Zink, ([18], Section 5). More precisely, one can use corollary 5.6 to describe deformations of filtered Barsotti-Tate groups with additional structures as successive extensions of the corresponding deformations of its Barsotti-Tate subquotients. Such observation reduces the proof of formal smoothness to the cases considered in [18] (see also [12], Lemma II.1.9 and Corollary II.1.10, pp. 71-73).

Finally, if $\mathcal{F}$ is formally smooth, then $\mathcal{F}^{\text {rig }}$ is a smooth rigid analytic space and thus also its étale coverings $\mathcal{F}^{U}$, for all $U \subseteq U_{0} \subset P\left(\mathbb{Q}_{p}\right)$.

4.3. - Similarly to the case of the moduli spaces introduced by Rapoport and Zink, we are interested in studying the limit of the $l$-adic étale cohomology groups of the rigid analytic spaces $\mathcal{F}^{U}$ as representations of $P\left(\mathbb{Q}_{p}\right) \times W_{E}$ and $J\left(\mathbb{Q}_{p}\right)$.

More precisely, let $l$ be a prime number, $l \neq p$, and $\hat{\bar{E}_{0}}$ the completion of an algebraic closure of $E_{0}$. For any level $U$, we consider the cohomology groups

$$
H^{i}\left(\mathcal{F}^{U}\right)=H_{c}^{i}\left(\mathcal{F}^{U} \times_{E_{0}} \hat{\bar{E}_{0}}, \mathbb{Q}_{l}\left(D_{\mathcal{F}}\right)\right)=\underline{\lim } \mathcal{V} H_{c}^{i}\left(\mathcal{V} \times_{E_{0}} \hat{\bar{E}_{0}}, \mathbb{Q}_{l}\left(D_{\mathcal{F}}\right)\right)
$$


where $\mathcal{V}$ varies among the open subspaces of $\mathcal{F}^{U}$ of finite type, and $D_{\mathcal{F}}=\operatorname{dim} \mathcal{F}^{U}(i \in \mathbb{N})$. On them, there is a natural action of $J\left(\mathbb{Q}_{p}\right)$ and $W_{E}$ induced by the action of $J\left(\mathbb{Q}_{p}\right)$ and the descent data over $E$ on the space $\mathcal{F}^{U}$.

As the level $U$ varies, these cohomology groups form a projective limit which is natural endowed with an action of $P\left(\mathbb{Q}_{p}\right)$ induced by the action of $P\left(\mathbb{Q}_{p}\right)$ on the tower $\mathcal{F}^{\infty}$. Moreover, as in the case of the cohomology of the Rapoport-Zink spaces, the following result on the cohomology of the system $\mathcal{F}^{\infty}$ holds.

Theorem 4.4. - Let $\rho$ be an admissible l-adic representation of $J\left(\mathbb{Q}_{p}\right)$.

1. The groups $H^{i, j}\left(\mathcal{F}^{\infty}\right)_{\rho}=\lim _{U} \operatorname{Ext}_{J\left(\mathbb{Q}_{p}\right)}^{j}\left(H^{i}\left(\mathcal{F}^{U}\right), \rho\right)$ vanish for almost all $i, j \geq 0$.

2. There is a natural action of $P\left(\mathbb{Q}_{p}\right) \times W_{E}$ on the $H^{i, j}\left(\mathcal{F}^{\infty}\right)_{\rho}$.

3. The representations $H^{i, j}\left(\mathcal{F}^{\infty}\right)_{\rho}$ are admissible.

Proof. - The same proof used in the case of the $\mathcal{M}^{U}$ 's in [22] (section 8.2) applies to these new moduli spaces.

We define a virtual representation of $P\left(\mathbb{Q}_{p}\right) \times W_{E}$

$$
H^{\bullet}\left(\mathcal{F}^{\infty}\right)_{\rho}=\sum_{i, j \geq 0}(-1)^{i+j} H^{i, j}\left(\mathcal{F}^{\infty}\right)_{\rho} .
$$

4.4. - We are interested in investigating how these new moduli spaces of Barsotti-Tate groups associated to the data $(B, V, b, \mu)$ (together with a choice of a compatible filtration on $V$ ) compare with the ones defined by Rapoport and Zink.

We already observed that there exists a natural morphism of $\mathcal{O}_{E_{0}}$-formal scheme $j: \mathcal{F} \rightarrow \mathcal{M}$, corresponding to forgetting the filtration on the Barsotti-Tate groups. It is clear from the definition that $j$ is equivariant for the descent data over $\mathcal{O}_{E}$ and for the action of the group $J\left(\mathbb{Q}_{p}\right)$. Moreover, for any level $U \subseteq U_{0} \subset G\left(\mathbb{Q}_{p}\right)$, by the universal properties of the spaces $\mathcal{M}^{U} \rightarrow \mathcal{M}^{U_{0}}$, there exists a compatible system of morphisms,

$$
j^{U}: \mathcal{F}^{U}=\mathcal{F}^{U \cap P\left(\mathbb{Q}_{p}\right)} \rightarrow \mathcal{M}^{U},
$$

lifting $j^{\text {rig }}$, which are equivariant for the descent data and the action of $J\left(\mathbb{Q}_{p}\right)$, and also for the action of $P\left(\mathbb{Q}_{p}\right) \subset G\left(\mathbb{Q}_{P}\right)$, as the level $U$ varies.

Furthermore, it is an easy consequence of the definition that the morphisms $\Theta^{U}: \mathcal{P}^{U} \rightarrow \mathcal{M}^{U}$ (introduced in section 3.8) factor through the morphisms $j^{U}: \mathcal{F}^{U} \rightarrow \mathcal{M}^{U}$, i.e. for all levels $U$

$$
\Theta^{U}: \mathcal{P}^{U} \rightarrow \mathcal{F}^{U} \rightarrow \mathcal{M}^{U}
$$

where the morphisms $\mathcal{P}^{U} \rightarrow \mathcal{F}^{U}$, which for simplicity we also denote by $\Theta^{U}$, send

$$
\begin{gathered}
(E L): \quad\left\{\left(H^{i}, \beta^{i},\left[\alpha^{i}\right]\right)\right\}_{i} \mapsto\left(H=\oplus_{i} H^{i}, H_{\bullet}, \beta=\oplus_{i} \beta^{i},\left[\alpha=\oplus_{i} \alpha^{i}\right]\right), \\
(P E L): \quad\left\{\left(H^{i}, \ell_{H^{i}}, \beta^{i},\left[\alpha^{i}\right]\right)\right\}_{i} \mapsto\left(H, H_{\bullet}, \ell_{H}=\oplus_{i} \ell_{H^{i}}, \beta,[\alpha]\right),
\end{gathered}
$$

where the filtration $H_{\bullet}$ of $H$ is defined as $H_{j}=\oplus_{1 \leq i \leq j} H^{i}$. Clearly, the above morphisms are equivariant for the descent data over $E$, the action of $J\left(\mathbb{Q}_{p}\right)$ and the action of $M\left(\mathbb{Q}_{p}\right) \subset$ $P\left(\mathbb{Q}_{p}\right)$ on the projective systems.

$4^{\text {e }}$ SÉRIE - TOME $41-2008-\mathrm{N}^{\mathrm{o}} 5$ 
Proposition 4.5. - For any level $U \subseteq U_{0} \subset P\left(\mathbb{Q}_{p}\right)$, there exists a morphism of $E_{0}$-rigid analytic spaces

$$
\Xi^{U}: \mathcal{F}^{U} \rightarrow \mathcal{P}^{U}=\mathcal{P}^{U \cap M\left(\mathbb{Q}_{p}\right)}
$$

which is $J\left(\mathbb{Q}_{p}\right)$-equivariant, commutes with the descent data and satisfies the equality $\Xi^{U} \circ \Theta^{U}=1_{\mathcal{P}^{U}}$.

Moreover, as the level $U$ varies, the morphisms $\Xi^{U}$ commute with the transition morphisms and with the action of $P\left(\mathbb{Q}_{p}\right)$ on the projective systems $\mathcal{F}^{\infty}$ and $\mathcal{P}^{\infty}$.

Proof. - We define the morphisms $\Xi^{U}$ by considering the graded pieces of the filtration on the Barsotti-Tate groups, together with the induced structures.

More precisely, given a triple $\left(H, H_{\bullet}, \beta\right) \in \mathcal{F}(S), S \in \operatorname{Nilp}_{\mathcal{O}_{E_{0}}}$, the subquotients $H^{i}=$ $H_{i} / H_{i-1}$ of $H$, for any $i=1, \ldots, t$, inherit an action of $\mathcal{O}_{B}$ (since this action preserves the filtration), and in the (PEL) case a compatible system of isomorphisms $\ell_{H^{i}}$ induced on the subquotients by the restrictions of the polarization $\ell_{H}$ of $H$ to the filtering subgroups. Similarly, the quasi-isogeny $\beta$ induces some quasi-morphisms $\beta^{i}: \Sigma_{\bar{S}}^{i} \rightarrow H_{\bar{S}}^{i}$. By induction on the length $t$ of the filtration of $\Sigma$, it is easy to check that, after multiplying by a sufficiently large power of $p$, the $\beta^{i}$ are well-defined morphisms which are surjective and have finite flat kernels. Thus, the subquotients $H^{i}$ are Barsotti-Tate groups and the quasi-morphisms $\beta^{i}$ quasi-isogenies. It is then easy to check that the pairs $\left(H^{i}, \beta^{i}\right), i=1, \ldots, t$, satisfy the required properties. Finally, any trivialization by $\Lambda$ of the local system $\mathcal{T}$ which preserves the filtrations $\Lambda_{\bullet}$ and $\mathcal{T}_{\bullet}$ induces trivializations of the subquotients $\mathcal{T}^{i}$ by $\Lambda^{i}$.

It follows from the definition that the morphisms $\Xi^{U}$ are equivariant for the descent data over $E$ and for the action of $J\left(\mathbb{Q}_{p}\right)$, and that they are also compatible with the natural projections, and with the action of $P\left(\mathbb{Q}_{p}\right)$ on $\mathcal{F}^{\infty}$ and $\mathcal{P}^{\infty}$, as the level $U$ varies. Finally, it is easy to see that they satisfy the equalities $\Xi^{U} \circ \Theta^{U}=1_{\mathcal{P} U}$, for all levels $U$.

Proposition 4.6. - For each level $U$, the morphism $\Theta^{U}: \mathcal{P}^{U} \rightarrow \mathcal{F}^{U}$ is a closed immersion.

Proof. - We first consider the case of level $U=U_{0}$. Let $\iota^{i}: \Sigma^{i} \rightarrow \Sigma$ denote the natural inclusions of the direct summands of $\Sigma$, and $\left(\mathcal{H}, \mathcal{H}_{\bullet}, \beta\right)$ denote the universal object over $\mathcal{F}$. Then, the image of the morphism $\Theta^{U_{0}}$ can be identified with the closed locus in $\mathcal{F}^{U_{0}}$ where the quasi-morphisms $\xi^{i}=\beta \circ \iota^{i} \circ\left(\beta^{i}\right)^{-1}: \mathcal{H}^{i}=\mathcal{H}_{i} / \mathcal{H}_{i-1} \rightarrow \mathcal{H}$ are morphisms, for all $i=1, \ldots, t$.

In the case of level $U \subseteq U_{0}$, let $s^{i}: \Lambda^{i} \rightarrow \Lambda$ (resp. $t^{i}: \mathcal{T}^{i} \rightarrow \mathcal{T}$ ) denote the natural inclusions corresponding to $\iota^{i}$ (resp. $\xi^{i}$ ), and write $\alpha_{\bullet}: \Lambda_{\bullet} \rightarrow \mathcal{T}_{\bullet}$ for the universal level structure over $\mathcal{F}^{U}$. Then, the image of the morphisms $\Theta^{U}$ is determined by the above conditions and by the further request that the morphisms $\alpha \circ s^{i}-t^{i} \circ \alpha^{i}: \Lambda^{i} \rightarrow \mathcal{T}_{i-1}$ vanish, for all $i$. The latter conditions are also represented by a unique closed subspace. Thus, the morphisms $\Theta^{U}$ are closed and it follows from Proposition 4.5 that they are actually closed immersions. 


\section{Properties of the slope filtration}

It follows from the definitions of the three systems of moduli spaces associated to the data $(B, V, b, \mu)$ that understanding the differences among them amounts to understanding certain questions regarding the slope filtration (and coarser ones) of a Barsotti-Tate group, e.g. when it exists, when it is split.

For clarity, we address all the pertinent questions on the properties of the slope filtration of Barsotti-Tate groups in this section, postponing the study of the moduli spaces of BarsottiTate groups we have introduced to latter sections. (For more details on the notion of a slope filtration of a Barsotti-Tate group, we refer to [10], [15],[33],[29].)

In the following, we consider the case of a general admissible pair $(b, \mu)$. We postpone to section 6 the discussion of stronger properties which are known to hold for pairs of (HN) type.

5.1. - We start by considering the case of a Barsotti-Tate group over a scheme $S$ of characteristic $p$. We remark that the following results hold for any filtration by Barsotti-Tate subgroups with subquotients of distinct slopes, i.e. for any choice of an order on the slopes.

Our first result regards the existence of a filtration.

Proposition 5.1. - We fix a Barsotti-Tate group over $k, \Sigma=\oplus_{i} \Sigma^{i}$ as in section 3.5, and write $\Sigma_{j}=\oplus_{0 \leq i \leq j} \Sigma^{i}(j=1, \ldots, t)$ for its filtration as in 4.1 .

Let $\mathcal{G}$ be a Barsotti-Tate group over a noetherian $k$-scheme $S$, together with a quasi-isogeny $\beta: \Sigma_{S} \rightarrow \mathcal{G}$.

Then there exists a stratification of $S$ by closed subschemes

$$
S=S_{0} \supseteq S_{1} \supseteq \cdots \supseteq S_{r} \supset S_{r+1}=\varnothing, \quad S_{s}^{\circ}=S_{s}-S_{s+1},
$$

such that:

1. for each $s$, the restriction of $\mathcal{G}$ to the locally closed subscheme $S_{s}^{\circ}$ admits an increasing filtration $\mathcal{G}_{0}$ satisfying the condition: for each $j=1, \ldots, t$, the restriction of $\beta$ to the subgroup $\Sigma_{j}$ induces a quasi-isogeny $\beta_{j}: \Sigma_{j} \rightarrow \mathcal{G}_{j}$;

2. for any connected scheme $Z$ and any morphism $f: Z \rightarrow S, f^{*} \mathcal{G}$ admits a filtration by Barsotti-Tate subgroups with the above property if and only if the morphism $f$ factors via the inclusion $S_{s}^{\circ} \hookrightarrow S$, for some $s \geq 0$.

Proof. - It is an easy observation that if a filtration $\mathcal{G}_{\bullet}$ of $\mathcal{G}$ satisfying the required properties exists, then it is unique. In fact, each subgroup $\mathcal{G}_{j}$ can be identified with the image of $\Sigma_{j} \subset \Sigma$ via $p^{n} \beta$, for $n$ a sufficiently large integer (namely, $n$ large enough for $p^{n} \beta$ to be an isogeny). On the other hand, a sufficient condition for the image of $\Sigma_{j} \subset \Sigma$ to be a BarsottiTate subgroup of $\mathcal{G}$ is that the finite group scheme $\operatorname{ker}\left(p^{n} \beta\right) \cap \Sigma_{j}$ is flat, for $n$ as above. (We remark that this condition does not depend on the choice of $n \gg 0$.) Furthermore, if we require that the restrictions of $\beta$ to the subquotients $\Sigma_{j}$ are quasi-isogenies then the above conditions are also necessary.

Thus, the existence of a stratification of $S$ as in the statement is a special case of Mumford's construction of the flattening stratification for a coherent sheaf over a noetherian scheme $S$, applied to the push-forward of the structure sheaf of $\operatorname{ker}\left(p^{n} \beta\right) \cap \Sigma_{j}$ (for each $j$ ). We recall this construction in the next lemma.

$4^{\text {e }}$ SÉRIE - TOME $41-2008-\mathrm{N}^{\mathrm{o}} 5$ 
Lemma 5.2 ([27]; [16], Proposition 6.4.3, p. 163). - Let $\mathcal{F}$ be a coherent sheaf over a noetherian scheme $S$ (not necessarily reduced). Then there exists a stratification of $S$ by closed subschemes (not necessarily distinct)

$$
S=S_{0} \supseteq S_{1} \supseteq \cdots \supseteq S_{r} \supset S_{r+1}=\varnothing
$$

with the universal property that for any scheme $Z$ and morphism $f: Z \rightarrow S, f^{*} \mathcal{F}$ is locally free of rank $i$ if and only if the morphism $f$ factors via the inclusion $S_{i}^{\circ}=S_{i}-S_{i+1} \hookrightarrow S$.

When $S$ is a reduced scheme, $S_{i}$ is simply the closed reduced subscheme of $S$ whose underlying space is the locus where the sheaf $\mathcal{F}$ has rank greater than or equal to $i$.

We remark that it follows from the conditions satisfied by the filtration $\mathcal{G}$. of $\mathcal{G}$, that the groups $\mathcal{G}^{i}=\mathcal{G}_{i} / \mathcal{G}_{i-1}$ are Barsotti-Tate groups and the quasi-isogenies $\beta_{j}$ induce quasiisogenies among the subquotients $\beta^{i}: \Sigma^{i} \rightarrow \mathcal{G}^{i}$, for all $i$. Thus, the $\mathcal{G}^{i}$ have all distinct slopes, and in particular, in the case when $\Sigma$ • is the slope filtration of $\Sigma$, the induced filtration $\mathcal{G}$. of $\mathcal{G}$ is its (unique) slope filtration. Finally, in the case of a Barsotti-Tate group endowed with additional structures, it follows from the fact that the subquotients have distinct slopes that the filtration is compatible with the additional structures (i.e. the subgroups $\mathcal{G}_{j}$ and the subquotients $\mathcal{G}^{i}$ inherits a $\mathcal{O}_{B}$-action and in the (PEL) case the polarization induces some isomorphisms $\left.\mathcal{G}^{i} \simeq\left(\mathcal{G}^{j}\right)^{\vee}\right)$.

In the following, we always assume that a filtration of a Barsotti-Tate group and a quasiisogeny between filtered Barsotti-Tate groups to satisfy the above conditions, namely that the filtration has Barsotti-Tate subquotients with distinct slopes and that the quasi-isogeny preserves the filtration and induces quasi-isogenies between the corresponding subquotients. We point out that the assumption on the filtration implies that a quasi-isogeny between filtered Barsotti-Tate groups is uniquely determined by the induced quasi-isogenies between the corresponding subquotients.

5.2. - Our next result regards the existence of a splitting of the induced filtration. We call a filtered Barsotti-Tate group split if its filtration is canonically split, i.e. $\mathcal{G}=\oplus_{i} \mathcal{G}^{i}$.

Proposition 5.3. - Let $\mathcal{G}$ be a filtered Barsotti-Tate group (with additional structures) defined over a noetherian $k$-scheme $S$, together with a quasi-isogeny $\beta: \Sigma_{S} \rightarrow \mathcal{G}$.

There exist some positive integers $0=r_{1} \leq r_{2} \leq \cdots \leq r_{t}$ (which in the (PEL) case are chosen satisfying the relations $r_{i}+r_{j}=r_{t}$ for all $i, j$ with $i+j=t+1$ ) such that the pullback of $\mathcal{G}$ by the isogenies $p^{r_{i}}$ on the subquotients $\mathcal{G}^{i}$ is a filtered Barsotti-Tate group with additional structures and is split.

Proof. - We start by observing that for any positive integers $r_{i}$ satisfying the above conditions, the Barsotti-Tate group $\mathcal{H}$, obtained from $\mathcal{G}$ via pullbacks, canonically inherits additional structures, as well as a quasi-isogeny of Barsotti-Tate groups with additional structures $\tilde{\beta}: \Sigma_{S} \rightarrow \mathcal{H}$. For simplicity, we prove this only in the case of when the length of the filtration of $\mathcal{G}$ is 2 , i.e. $0 \rightarrow \mathcal{G}_{1} \rightarrow \mathcal{G} \rightarrow \mathcal{G}^{2} \rightarrow 0$. (The essential ideas of the proof are well represented by this case.) In general, the statement can be proved by induction on the length of the filtration $t$. (The case $t=1$ is obvious.)

For $t=2$, we have $\mathcal{H}=\mathcal{G} \times{ }_{\mathcal{G}^{2}} \mathcal{G}^{2}$ the pullback of $\mathcal{G}$ via the morphism $p^{r}: \mathcal{G}^{2} \rightarrow \mathcal{G}^{2}$, for some $r \geq 1$. Using the universal property of products, for each $\gamma \in \mathcal{O}_{B}$ we define 
$\gamma_{\mathcal{H}}=\left(\gamma_{\mathcal{G}}, \gamma_{\mathcal{G}^{2}}\right)$, where $\gamma_{\mathcal{G}}$ and $\gamma_{\mathcal{G}^{2}}$ denote the action of $\gamma$ on $\mathcal{G}$ and $\mathcal{G}^{2}$, respectively. In order to check that the morphism is well-defined it is enough to remark that the projection $\mathcal{G} \rightarrow \mathcal{G}^{2}$ and $p^{r}: \mathcal{G}^{2} \rightarrow \mathcal{G}^{2}$ are $\mathcal{O}_{B}$-linear morphisms. Finally, we need to verify that this construction indeed defines an action of $\mathcal{O}_{B}$ on $\mathcal{H}$, and this follows from the analogous properties for $\mathcal{G}$ together with the equalities of quasi-isogenies $\gamma_{\mathcal{H}}=\pi^{-1} \circ \gamma_{\mathcal{G}} \circ \pi$, for all $\gamma \in \mathcal{O}_{B}$, where $\pi: \mathcal{H} \rightarrow \mathcal{G}$ denotes the natural projection (which is an isogeny).

In the (PEL) case, we define the polarization $\ell_{\mathcal{H}}$ as follows. For $t=2$, we have $\mathcal{H}^{\vee}=$ $\left(\mathcal{G}^{2}\right)^{\vee} \amalg_{\left(\mathcal{G}^{2}\right)^{\vee}} \mathcal{G}^{\vee}$ the pushforward of $\mathcal{G}^{\vee}$ via multiplication by $p^{r}$ on $\left(\mathcal{G}^{2}\right)^{\vee}$. (This follows from the definition of $\mathcal{H}$ and the fact that the dual of the multiplication by $p^{r}$ is the multiplication by $p^{r}$ on the dual). Equivalently, given the short exact sequence $0 \rightarrow\left(\mathcal{G}^{2}\right)^{\vee} \rightarrow \mathcal{G}^{\vee} \rightarrow$ $\mathcal{G}_{1}^{\vee} \rightarrow 0, \mathcal{H}^{\vee}$ can also be realized as $\mathcal{H}^{\vee}=\mathcal{G}^{\vee} \times \mathcal{G}_{1}^{\vee} \mathcal{G}_{1}^{\vee}$ the pushforward of $\mathcal{G}$ via multiplication by $p^{r}$ on $\mathcal{G}_{1}^{\vee}$. We define $\ell_{\mathcal{H}}=\left(\ell_{\mathcal{G}}, \ell^{2}\right)$, for $\ell_{\mathcal{G}}$ the polarization of $\mathcal{G}$ and $\ell^{2}: \mathcal{G}^{2} \rightarrow \mathcal{G}_{1}^{\vee}$ the isomorphism induced by $\ell_{\mathcal{G}}$ on the quotients. The fact that $\ell_{\mathcal{H}}$ is a $\mathcal{O}_{B}$-linear polarization follows for the analogous properties of $\ell_{\mathcal{G}}$ and the equality $\ell_{\mathcal{H}}=c \cdot\left(\pi^{\vee} \circ \ell_{\mathcal{G}} \circ \pi\right)$, for some $c \in \mathbb{Q}_{p}^{\times}$.

Finally, we define the quasi-isogeny $\tilde{\beta}: \Sigma_{S} \rightarrow \mathcal{H}$ as $\tilde{\beta}=\pi^{-1} \circ \beta$. It follows from its definition that it is a quasi-isogeny of Barsotti-Tate groups with additional structures.

We are now left with the task of verifying that, for some integers $r_{i}$ as above, the BarsottiTate group $\mathcal{H}$ is split. We prove the statement by induction on $t$, the length of the filtration. For $t=1$ the statement is obvious $\left(r_{1}=0\right)$. For $t>1$, we treat the (EL) and (PEL) cases separately.

In the (EL) case, we consider the short exact sequence

$$
0 \rightarrow \mathcal{G}_{1} \rightarrow \mathcal{G} \rightarrow \mathcal{G}^{\prime} \rightarrow 0,
$$

where the Barsotti-Tate group $\mathcal{G}^{\prime}$ is endowed with a filtration of length $t-1$, of subquotients $\mathcal{G}^{i}, i=2, \ldots, t$.

By induction, there exist some integers $0=r_{2}^{\prime} \leq r_{3}^{\prime} \leq \cdots \leq r_{t}^{\prime}$ such that the pullback $\psi^{\prime}: \mathcal{H}^{\prime} \rightarrow \mathcal{G}^{\prime}$ of $\mathcal{G}^{\prime}$, by the isogenies $p^{r_{i}^{\prime}}$ on the subquotients $\mathcal{G}^{i}$, is split, i.e. $\mathcal{H}^{\prime}=\oplus_{i \geq 2} \mathcal{G}^{i}$.

We define $\psi: \tilde{\mathcal{H}} \rightarrow \mathcal{G}$ the pullback of $\mathcal{G}$ via the isogeny $\psi^{\prime}: \mathcal{H}^{\prime} \rightarrow \mathcal{G}^{\prime}$, regarded as an extension of $\mathcal{H}^{\prime}$ by $\mathcal{G}_{1}$. Then, in order to conclude it suffices to prove that there exists an integer $r \geq 0$ such that the pullback $\Psi: \mathcal{H} \rightarrow \tilde{\mathcal{H}}$ of $\tilde{\mathcal{H}}$ by the isogeny $p^{r}$ on $\mathcal{H}^{\prime}$ is split. (This is equivalent to the statement for integers $0=r_{1} \leq r_{2} \leq \cdots \leq r_{t}, r_{j}=r_{j}^{\prime}+r$ for all $j=2, \ldots, t$.)

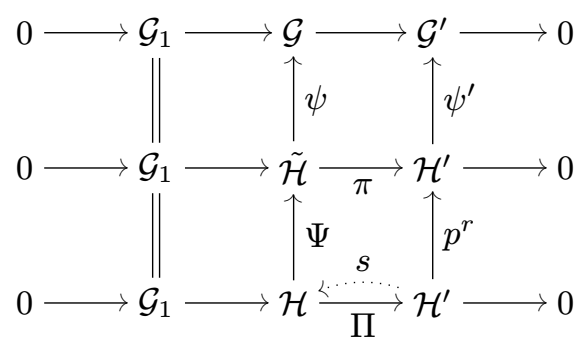

We write $\tilde{\beta}=\psi^{-1} \circ \beta: \Sigma_{S} \rightarrow \tilde{\mathcal{H}}$, and $\tilde{\beta}^{\prime}: \Sigma_{S}^{\prime}=\oplus_{i \geq 2} \Sigma_{S}^{i} \rightarrow \mathcal{H}^{\prime}$ for the quasi-isogeny induced by $\tilde{\beta}$ between the quotients. Without loss of generality, we may assume $\tilde{\beta}$ is an isogeny. 
We choose $r \geq \operatorname{deg} \tilde{\beta}^{\prime}$, i.e. such that the quasi-isogeny $p^{r}\left(\tilde{\beta}^{\prime}\right)^{-1}: \mathcal{H}^{\prime} \rightarrow \Sigma^{\prime}$ is an isogeny. We claim that $\mathcal{H}=\left(1, p^{r}\right)^{*} \tilde{\mathcal{H}}$ is split. Indeed, let $\iota^{\prime}: \Sigma^{\prime} \rightarrow \Sigma$ denote the inclusion corresponding to the splitting $\Sigma=\Sigma_{1} \oplus \Sigma^{\prime}$. We define a morphism

$$
\phi=\tilde{\beta} \circ \iota^{\prime} \circ p^{r}\left(\tilde{\beta}^{\prime}\right)^{-1}: \mathcal{H}^{\prime} \rightarrow \tilde{\mathcal{H}} .
$$

Then, the pair $\left(\phi, \mathbb{I}_{\mathcal{H}^{\prime}}\right)$ satisfies the equality $\pi \circ \phi=p^{r}$, and thus defines a morphism $s: \mathcal{H}^{\prime} \rightarrow \mathcal{H}$ which it is easy to check is a section of $\Pi$.

Finally, the (PEL) case follows from the (EL) case, by remarking that it is possible to enlarge the differences $r_{i+1}-r_{i}$ so that the integers $r_{i}$ satisfy the additional property $r_{i}+r_{j}=r_{t}$ for all $i, j$ with $i+j=t+1$.

We remark that in the case when the filtration of $\Sigma$ is associated to the increasing order on the slopes, it is possible to choose the integers $r_{i}$ independently of the degree of $\beta$ (see [31], Lemma 2.19, p. 56). On the other hand, for any field extension $K$ of $k$ and any Barsotti-Tate group $G / K$, there always exists an isogeny $\beta: \Sigma_{K} \rightarrow G$ of degree less than or equal to a uniform bound $r$ depending only on the height of $\Sigma$ (see [21], Sections (3.4) and (3.5), p. 44).

5.3. - We now move our focus to the existence and properties of a filtration lifting the slope filtration to characteristic zero.

We start by remarking the following weak analogue of proposition 5.3 for a filtered Barsotti-Tate group over a general base.

Proposition 5.4. - Let $\mathcal{G}$ be a filtered Barsotti-Tate group (with additional structures) over a scheme $S$. Then, for any integer $m \geq 0$, let $\mathcal{H}=\mathcal{H}_{m}$ be the pullback of $\mathcal{G}$ under the isogeny $p^{(i-1) m}$ of $\mathcal{G}^{i}$, for all $i=1, \ldots, t$. Then, $\mathcal{H}$ is also a filtered Barsotti-Tate group with additional structures and moreover there exists a canonical isomorphism

$$
\mathcal{H}\left[p^{m}\right] \simeq \oplus_{i} \mathcal{G}^{i}\left[p^{m}\right]
$$

i.e. the induced filtration of the $p^{m}$-torsion subgroups is split.

Proof. - We remark that it follows from the same arguments at the beginning of the proof of proposition 5.3 that the pullback $\mathcal{H}=\mathcal{H}_{m}$ inherits the additional structures on $\mathcal{G}$. Thus, it suffices to check that the induced filtration on $\mathcal{H}\left[p^{m}\right]$ is split.

We prove the statement by induction on the length of the filtration. For $t=1$ the statement is trivial.

For $t>1$, let us consider the short exact sequence

$$
0 \rightarrow \mathcal{G}_{1} \rightarrow \mathcal{G} \rightarrow \mathcal{G}^{\prime} \rightarrow 0 .
$$

Then $\mathcal{G}^{\prime}$ is a Barsotti-Tate group with a filtration of length $t-1$, and by inductive hypothesis the $p^{m}$-torsion subgroup of its pullback by the isogenies $p^{(i-2) m}$ on the subquotients $\mathcal{G}^{i}(i=$ $2, \ldots, t)$ is split. Let us denote this pullback by $\phi: \mathcal{H}^{\prime} \rightarrow \mathcal{G}^{\prime}$ and replace $\mathcal{G}$ by its pullback by $\phi, \phi^{*} \mathcal{G}$. Then, $\mathcal{H}=\left(1, p^{m}\right)^{*}\left(\phi^{*} \mathcal{G}\right)$, the pullback of $\phi^{*} \mathcal{G}$ by the isogeny $p^{m}$ on $\mathcal{H}^{\prime}$. We denote by $\Pi: \mathcal{H} \rightarrow \phi^{*} \mathcal{G}$ the associated isogeny. It is straightforward that the projection $\Psi: \mathcal{H} \rightarrow \mathcal{H}^{\prime}$ induces an isomorphism ker $\Pi \simeq \mathcal{H}^{\prime}\left[p^{m}\right]$, and thus a splitting

$$
\mathcal{H}\left[p^{m}\right] \simeq \mathcal{G}_{1}\left[p^{m}\right] \oplus \mathcal{H}^{\prime}\left[p^{m}\right] \simeq \oplus_{i} \mathcal{G}^{i}\left[p^{m}\right]
$$


Finally, in the next results, we address the questions regarding the possibility of lifting a filtration from characteristic $p$ to characteristic zero, and the uniqueness of such a lifting.

Let us first recall a result of Drinfeld on lifting quasi-isogenies of Barsotti-Tate groups. (This result originally appeared in [7].)

Proposition 5.5. - Let $\mathcal{G}, \mathcal{H}$ be two Barsotti-Tate groups over a scheme $S$, with p locally nilpotent. Let $S_{0} \subset S$ be a nilpotent immersion.

Then

$$
\operatorname{Hom}_{S}(\mathcal{G}, \mathcal{H}) \otimes_{\mathbb{Z}_{p}} \mathbb{Q}_{p} \rightarrow \operatorname{Hom}_{S_{0}}\left(\mathcal{G}_{S_{0}}, \mathcal{H}_{S_{0}}\right) \otimes_{\mathbb{Z}_{p}} \mathbb{Q}_{p}
$$

is bijective.

The following two results are an immediate consequence of the above proposition.

Corollary 5.6. - Let $\mathcal{G}$ be a Barsotti-Tate group defined over a scheme $S$, and $S_{0} \subset S$ a nilpotent immersion.

Suppose $\mathcal{G}$ is endowed with two filtrations $\mathcal{G}_{\bullet}$ and $\mathcal{H}_{\bullet}$ whose restriction to $S_{0}$ agrees.

Then the two filtrations agree, i.e. $\mathcal{G}_{\bullet}=\mathcal{H}_{\bullet}$.

Proof. - We prove the statement by induction on the length of the filtration, $t$. The case $t=1$ is trivial. For $t>1$, we consider the two short exact sequences

$$
0 \rightarrow \mathcal{G}_{1} \rightarrow \mathcal{G} \rightarrow \mathcal{G}^{\prime} \rightarrow 0 \quad \text { and } \quad 0 \rightarrow \mathcal{H}_{1} \rightarrow \mathcal{G} \rightarrow \mathcal{H}^{\prime} \rightarrow 0
$$

associated with the first subgroups in the filtrations $\mathcal{G}_{\bullet}$ and $\mathcal{H}_{\bullet}$, respectively.

Then, the identities of $\mathcal{G}_{1, S_{0}}=\mathcal{H}_{1, S_{0}}$ and $\mathcal{G}_{S_{0}}^{\prime}=\mathcal{H}_{S_{0}}^{\prime}$ lift uniquely to some quasi-isogenies $\mathcal{G}_{1} \rightarrow \mathcal{H}_{1}$ and $\mathcal{G}^{\prime} \rightarrow \mathcal{H}^{\prime}$, commuting with the identity on $\mathcal{G}$. It follows that the quasi-isogeny $\mathcal{G}_{1} \rightarrow \mathcal{H}_{1}$ is an isogeny, thus an isomorphism, and that the same holds for $\mathcal{G}^{\prime} \rightarrow \mathcal{H}^{\prime}$.

It is clear that, in the case of Barsotti-Tate groups with additional structures, the fact that the underlying filtration of Barsotti-Tate groups agrees suffices to deduce that the induced additional structures on the subgroups also agree.

COROLlaRY 5.7. - Let $\mathcal{G}$ be a Barsotti-Tate group with additional structures over a scheme $S$, and $S_{0} \subset S$ a nilpotent immersion.

Assume $\mathcal{G}_{S_{0}}$ is also endowed with a filtration and that this filtration is compatible with the additional structures (in the usual sense).

Then, if there exists a filtration on $\mathcal{G}$ lifting that on $\mathcal{G}_{S_{0}}$, then it is compatible with the additional structures.

Proof. - The proof of this corollary is analogous to the previous one (here in place of the identity we consider the isogenies associated with the additional structures).

Finally, we consider the problem of lifting the slope filtration (and coarser ones) from characteristic $p$ to characteristic 0 .

$4{ }^{\mathrm{e}}$ SÉRIE - TOME $41-2008-\mathrm{N}^{\circ} 5$ 
Proposition 5.8. - Let $S$ be a p-adic formal scheme (resp. $S=\operatorname{Spec} A$ for $A$ a noetherian ring with $J$ an ideal of $A$ which contains some power of $p$ and satisfies $J^{2}=(0)$ ), and write $\bar{S}=V(p)($ resp. $\bar{S}=\operatorname{Spec} A / J)$. Let $\mathcal{G}$ be a Barsotti-Tate group over $S$ and suppose $G=\mathcal{G}_{\bar{S}}$ is endowed with a filtration.

Then there exists a unique closed formal subscheme (resp. closed subscheme) $W$ of $S$, $W \supset \bar{S}$, such that for any p-adic formal scheme (resp. any scheme $Z$ ) and any morphism $f: Z \rightarrow S, f^{*} \mathcal{G}$ admits a filtration by Barsotti-Tate groups lifting the given filtration of $\bar{f}^{*} G$ ( for $\bar{f}=f_{\mid \bar{S}}$ ) if and only if the morphism $f$ factors via the inclusion $W \hookrightarrow S$.

Proof. - We prove the statement by induction on the length of the filtration, $t$. For $t=1$, the statement is trivial. For $t>1$, we consider the short exact sequence

$$
0 \rightarrow G_{t-1} \rightarrow G \rightarrow G^{t} \rightarrow 0
$$

corresponding to the last step in the filtration. For simplicity, we rewrite $G_{t-1}=G_{1}$, $G^{t}=G_{2}$ and regard $G$ as an extension of $G_{2}$ by $G_{1}$.

Since the inclusion $\bar{S} \subset S$ has a canonical divided power structure, we may apply the theory of Grothendieck-Messing. Let $M$ (resp. $M_{1}, M_{2}$ ) denote the crystal of the BarsottiTate group $G$ (resp. $G_{1}, G_{2}$ ), it is a locally free $\mathcal{O}_{S}$-module of finite rank, its rank equal to the height of the Barsotti-Tate group. Moreover, there is a short exact sequence of $\mathcal{O}_{S}$-modules

$$
0 \rightarrow M_{1} \rightarrow M \rightarrow M_{2} \rightarrow 0 \text {. }
$$

Let $L=\operatorname{Lie}(G)$ (resp. $L_{1}=\operatorname{Lie}\left(G_{1}\right), L_{2}=\operatorname{Lie}\left(G_{2}\right)$ ) denote the Lie algebra of $G$ (resp. $G_{1}, G_{2}$ ). For any $*=\varnothing, 1,2, L_{*}$ is a locally free $\mathcal{O}_{\bar{S}}$-module, of rank equal to the dimension of the Barsotti-Tate group, equipped with an epimorphism of $\mathcal{O}_{\bar{S}}$-modules, $\pi_{*}: M_{*} \otimes_{\mathcal{O}_{S}} \mathcal{O}_{\bar{S}} \rightarrow L_{*}$. Moreover, there is a short exact sequence of $\mathcal{O}_{\bar{S}}$-modules

$$
0 \rightarrow L_{1} \rightarrow L \rightarrow L_{2} \rightarrow 0
$$

which is compatible under the epimorphisms $\pi_{*}$, with the short exact sequence of crystals.

Then, the datum of a Barsotti-Tate group $\mathcal{G} / S$ lifting $G$ is equivalent to the datum of a locally free $\mathcal{O}_{S}$-quotient $\varpi: M \rightarrow \mathcal{L}$ lifting $\pi: M \otimes \mathcal{O}_{\bar{S}} \rightarrow L$ (namely, $\mathcal{L}=\operatorname{Lie}(\mathcal{G})$ ). Moreover, choosing a lifting of the sequence $0 \rightarrow G_{1} \rightarrow G \rightarrow G_{2} \rightarrow 0$ to $\mathcal{G}$ is equivalent to choosing a lifting of the sequence $0 \rightarrow L_{1} \rightarrow L \rightarrow L_{2} \rightarrow 0$ to $\mathcal{L}$, compatible with the short exact sequence of crystals.

We define $\mathcal{L}_{2}$ to be the $\mathcal{O}_{S}$-module $\mathcal{L} / \varpi\left(M_{1}\right)$, regarded as quotient of $M_{2}$. Then, $L_{2}=$ $\mathcal{L}_{2} \otimes_{\mathcal{O}_{S}} \mathcal{O}_{\bar{S}}$ and in particular $\mathcal{L}_{2}$ has rank less than or equal to $r=\operatorname{rk}\left(L_{2}\right)$.

Following Lemma 5.2, we define $W=S_{r}$ the closed subscheme of $S$ where $\mathcal{L}_{2}$ is locally free of rank $r=\operatorname{rk} L_{2}$. In particular, $W \supset \bar{S}$.

For any scheme $Z$ and any morphism $f: Z \rightarrow S$, the existence of a filtration on $f^{*} \mathcal{G}$ lifting the filtration of $\bar{f}^{*} G$ implies that $f^{*} \mathcal{L}_{2}=\mathcal{L}_{2} \otimes_{\mathcal{O}_{S}} \mathcal{O}_{Z}$ is locally free, of rank equal to $r k\left(L_{2}\right)$. Thus in particular, $f$ factors via the inclusion $W \subset S$, and this condition is also sufficient for the existence of a filtration.

In the case of Barsotti-Tate groups with additional structure (and $S$ an $\mathcal{O}_{E}$-scheme), the hypothesis that the moduli data are unramified implies that the associated (polarized) filtered crystals are locally free $\mathcal{O}_{S} \otimes_{\mathcal{O}_{E}} \mathcal{O}_{B}$-modules. In particular, one defines $W$ according to the $\mathcal{O}_{S} \otimes_{\mathcal{O}_{E}} \mathcal{O}_{B}$-rank of $\mathcal{L}_{2}$. 


\section{Properties of the Hodge-Newton filtration}

In this section, we focus on those decompositions $V=\oplus_{i} V^{i}$ of $V$ which arise from Hodge-Newton decompositions of the associated isocrystals $N_{b}(V)$, i.e. associated with admissible pairs of (HN) type. For these decompositions, many of the results of Section 5 can be improved.

6.1. - In the general context of $F$-crystals (not necessarily arising from Barsotti-Tate groups), results on the existence of Hodge-Newton decompositions and filtrations are due to Katz. In the case over a perfect field of positive characteristic, in [15] (Theorem 1.6.1, p. 133) Katz proves that if a $F$-crystal has a break-point of its Newton polygon which lies on its Hodge polygon, then it decomposes uniquely as direct sum of two $F$-crystals, its Hodge (resp. Newton) polygon formed by joining end-to-end the Hodge (resp. Newton) polygons of its constituents, with the break-point as the joining point. When specialized to the case of crystals of Barsotti-Tate groups, Katz's result recovers the existence of the multiplicativebilocal-étale decomposition for Barsotti-Tate groups ([6], Section IV.8, Proposition p. 92). Indeed, in the case of a crystal of a Barsotti-Tate group the Newton polygon has slopes between 0 and 1, while the possible values for the slopes of the Hodge polygon are only 0 and 1. Therefore, if a break-point of the Newton polygon of a Barsotti-Tate group lies on the Hodge polygon, then the two polygons necessarily share a side, of slope either 0 or 1. We remark that since in this case the Hodge polygon coincides with the ordinary Newton polygon, it follows that a break-point of the Newton polygon lies on the Hodge polygon if and only if it is of (HN) type. Moreover, in this context, in [25] (Proposition 4.9, p. 69) Messing proved that the multiplicative-bilocal-étale decomposition lifts uniquely to a filtration in characteristic zero.

More recently, in [20], Kottwitz has extended Katz's theorem to the case of $F$-crystals over a perfect field, which are endowed with additional structures by an unramified group. (We remark that the results in [20] are not stated in full generality, but the same ideas lead to more comprehensive statements, see [24].) In the following, we recall this result in the case of crystals associated with Barsotti-Tate groups with additional structures, and also report on a result on the existence of a lifting of the Hodge-Newton filtration to characteristic zero that is proved in [24], which generalizes Messing's theorem to this context.

Finally, we point out that in the case of $\mu$-ordinary Barsotti-Tate groups, the existence of the Hodge-Newton decomposition and filtration was already known due to the work of Moonen in [26] (Corollary 1.3.12 and Proposition 2.1.9). In that case, similarly to the classical ordinary case, the Hodge-Newton decomposition coincides with the slope decomposition.

6.2. - We maintain the notations we introduced in Section 1.2. Given the moduli data $(B, V)$, for any admissible pair $(b, \mu)$, let $V=\oplus_{s} W^{s}$ be the decomposition of $V$ underlying to the slope decomposition of the isocrystal $N_{b}(V)$, ordered accordingly to the increasing order on the slopes. Then, for each break-point $x$ of the Newton polygon $\nu_{b}$ of $N_{b}(V)$ (which is not an end-point), the associated (non-trivial) $B$-linear decomposition $V=V^{1} \oplus V^{2}$ can be described as follows. If $x$ is the $N$-th break-point after the origin $(N \geq 1)$, then

$$
V^{1}=\oplus_{i \leq N} W^{i} \text {, and } V^{2}=\oplus_{i>N} W^{i} .
$$

$4^{\mathrm{e}}$ SÉRIE - TOME $41-2008-\mathrm{N}^{\mathrm{o}} 5$ 
Similarly, in the (PEL) case, the (non-trivial) symplectic $B$-linear decomposition $V=V_{1} \oplus$ $V_{2} \oplus V_{3}$ associated with a symmetrical pair of break-points $(x, \hat{x})$ (not necessarily distinct) of $\nu_{b}$ (which are not the end-points), can be described as follows. If $x, \hat{x}$ are the $N$-th and $t+1-N$-th break-points after the origin, $1 \leq N \leq t / 2$, then

$$
V^{1}=\oplus_{1 \leq i \leq N} W^{i}, \quad V^{2}=\oplus_{N+1 \leq i \leq t-N} W^{i}, \text { and } V^{3}=\oplus_{t+1-N \leq i \leq t} W^{i} .
$$

We remark that, in the (PEL) case, a break-point $x$ of $\nu_{b}$ lies on the $\mu$-ordinary polygon if and only if its symmetric one $\hat{x}$ does.

Finally, we recall the following definition.

Definition 6.1. - We say that $b \in B(G, \mu)$ (or the corresponding pair $(b, \mu)$ ) is of (HN) type if there exists a break-point $x$ of the Newton polygon of the isocrystal $N_{b}(V)$ which lies on the $\mu$-ordinary polygon, and the two polygons coincide up to $x$ or from $x$ on. We call such a break-point $x$ of $(\mathrm{HN})$ type.

We remark that, in the (PEL) case, a break-point $x$ is of (HN) type if and only if its symmetric one $\hat{x}$ is. Indeed the two polygons coincide up to $x$ (resp. from $x$ on) if and only if they coincide from $\hat{x}$ on (resp. up to $\hat{x}$ ).

Theorem 6.2 ([20], Theorem 4.1, p. 1442; [24], Corollary 7, p. 5)

Let $G=\operatorname{Res}_{F / \mathbb{Q}_{p}} G_{0}$ for $F / \mathbb{Q}_{p}$ a finite unramified field extension and $G_{0}=G L_{n}$ or $G S p_{2 n}$, $n \geq 1$.

Let $k$ be a perfect field, of characteristic $p$, and $H / k$ a Barsotti-Tate group with $G$-structures.

Suppose that there exists a break-point $x$ of the Newton polygon of $H$ which lies on the associated $\mu$-ordinary polygon.

Then, there exists a unique decomposition of $H$ as direct sum

$$
H=H^{1} \oplus H^{2}, \text { resp. in the (PEL) case } H=H^{1} \oplus H^{2} \oplus H^{3},
$$

with $H^{1}$ and $H^{2}$, resp. $H^{1}, H^{2}$ and $H^{3}$, Barsotti-Tate groups with additional structures, such that the corresponding decomposition of the isocrystal of $H, N(H)=N\left(H^{1}\right) \oplus N\left(H^{2}\right)$, resp. $N(H)=N\left(H^{1}\right) \oplus N\left(H^{2}\right) \oplus N\left(H^{3}\right)$, is the decomposition associated with $x$, resp. in the (PEL) case with the symmetric pair $(x, \hat{x})$.

Theorem 6.3 (see [24], Theorem 10, p. 5). - Let $G$ as in 6.2. Let $R$ be a complete local ring over $\mathcal{O}_{E}$ with perfect residue field $k$, of characteristic $p$, and $\mathcal{H} / R$ a Barsotti-Tate group with $G$-structures.

Suppose that there exists a break-point $x$ of the Newton polygon of $\mathcal{H}$ which is of $(H N)$ type.

Then, there exists a unique filtration of $\mathcal{H}$ by Barsotti-Tate subgroups

$$
\mathcal{H}_{1} \subset \mathcal{H} \text {, resp. in the }\left(\text { PEL) case } \mathcal{H}_{1} \subset \mathcal{H}_{2} \subset \mathcal{H},\right.
$$

with $\mathcal{H}_{1}=\mathcal{H}^{1}$ and $\mathcal{H}^{2}=\mathcal{H} / \mathcal{H}_{1}$, resp. $\mathcal{H}_{1}=\mathcal{H}^{1}, \mathcal{H}^{2}=\mathcal{H}_{2} / \mathcal{H}_{1}$ and $\mathcal{H}^{3}=\mathcal{H} / \mathcal{H}_{2}$ BarsottiTate groups with additional structures, such that its restriction to the special fiber $H=\mathcal{H}_{k}$ is canonically split and agrees with decomposition associated with $x$ (resp. with $(x, \hat{x})$ ) by Theorem 6.2 . 
6.3. - In the following, to any admissible pair $(b, \mu)$ of (HN) type we associate the unique decomposition (and corresponding filtration) of $V$ which is the common refinement of the decompositions (resp. filtrations) associated with the break-points of (HN) type of $\nu_{b}$. We call the corresponding decomposition of $N_{b}(V)$ its Hodge-Newton decomposition. In the cases when all the break-points of $\nu_{b}$ are of (HN) type (e.g. when $b$ is $\mu$-ordinary) it coincides with the slope decomposition.

\section{From the Levi subgroup to the associated parabolic}

In this section we compare the two tower of moduli spaces $\mathcal{P}^{\infty}$ and $\mathcal{F}^{\infty}$. Our goal is the proof of the following theorem.

THeOREM 7.1. - For any l-adic admissible representation $\rho$ of $J\left(\mathbb{Q}_{p}\right)$, we have an equality of virtual representations of $P\left(\mathbb{Q}_{p}\right) \times W_{E}$ :

$$
H^{\bullet}\left(\mathcal{F}^{\infty}\right)_{\rho}=H^{\bullet}\left(\mathcal{P}^{\infty}\right)_{\rho} .
$$

In particular, we focus on the study of the morphism $\Xi: \mathcal{F}^{\infty} \rightarrow \mathcal{P}^{\infty}$ (which we introduced in Proposition 4.5) and on the map that it induces between the cohomology groups, $\left(\Xi^{*}\right)^{\vee}$.

7.1. - We start by considering the morphisms of formal $\mathcal{O}_{E_{0}}$-schemes $\Xi: \mathcal{F} \rightarrow \mathcal{P}$ and $\Theta: \mathcal{P} \rightarrow \mathcal{F}$, satisfying the condition $\Xi \circ \Theta=\mathbb{I}_{\mathcal{P}}$.

In the following, for any formal scheme $X$ over $\mathcal{O}_{E_{0}}$ (resp. morphism $f: X \rightarrow Y$ of formal $\mathcal{O}_{E_{0}}$-scheme) we write $X_{0}=V\left(I_{X}\right)$, for $I_{X}$ a maximal ideal of definition of $X$ containing $p$, regarded as a $k$-scheme (resp. $f_{0}: X_{0} \rightarrow Y_{0}$ for the morphism of $k$-scheme obtained by restriction, for $f^{*} I_{Y} \subset I_{X}$ ), and $X_{\eta}$ (resp. $f_{\eta}$ ) for its rigid analytic fiber, regarded as a $E_{0}$-space (resp. as a morphism of $E_{0}$-spaces).

Let $\mathcal{Y}$ be the formal completion of $\mathcal{F}$ along $\mathcal{Y}_{0}=\Theta\left(\mathcal{P}_{0}\right) \subset \mathcal{F}_{0}$. Then, the morphism $\Theta: \mathcal{P} \rightarrow \mathcal{F}$ factors via the inclusion $j: \mathcal{Y} \hookrightarrow \mathcal{F}$. We write $\Xi^{\mathcal{Y}}$ for the restriction of $\Xi$ to $\mathcal{Y}$.

Proposition 7.2. - There exist some integers $0=r_{1} \leq r_{2} \leq \cdots \leq r_{t}$ (in the (PEL) case satisfying the conditions $r_{i}+r_{j}=r_{t}$ for all $i, j$ with $i+j=t+1$ ) and a morphism $\delta: \mathcal{F} \rightarrow \mathcal{Y}$, which is equivariant for the action of $J\left(\mathbb{Q}_{p}\right)$ and for the descent data, such that the following diagram commutes, for $\gamma=\left(1, p^{-r_{2}}, \ldots, p^{-r_{t}}\right) \in P\left(\mathbb{Q}_{p}\right)$ and $U^{\prime} \subseteq U_{r_{t}}$.

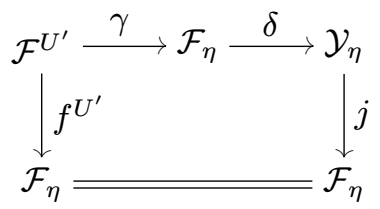

Proof. - We define a morphism $\delta: \mathcal{F} \rightarrow \mathcal{Y}$ as follows.

Let $Z_{0} \subset \mathcal{F}_{0}$ be a closed subscheme of finite type such that $\mathcal{F}_{0}=\cup_{t \in J\left(\mathbb{Q}_{p}\right)} t Z_{0}$. E.g. we can choose $Z_{0}$ to be the locus where the quasi-isogeny $\beta$ is an isogeny of degree less than or equal to $n$, for some integer $n$ sufficiently large. We write $Z$ for the formal completion of $\mathcal{F}$ along $Z_{0}$ (thus $\mathcal{F}=\cup_{t \in J\left(\mathbb{Q}_{p}\right)} t Z$ ). 
We first define a morphism $\delta: Z \rightarrow \mathcal{Y}$, and we then remark that it extends (in a unique way) to a $J\left(\mathbb{Q}_{p}\right) \times W_{E}$-equivariant morphism $\delta: \mathcal{F} \rightarrow \mathcal{Y}$ which makes the above diagram commute.

Let $\mathcal{H}$ denote the universal filtered Barsotti-Tate group over $\mathcal{F}$ restricted to $Z$, and $\beta: \Sigma_{Z_{0}} \rightarrow \mathcal{H}_{Z_{0}}$ the associated quasi-isogeny. Then, by Proposition 5.3, there exist some integers $0=r_{1} \leq r_{2} \leq \cdots \leq r_{t}$ (as in the statement) such that the pullback of $\mathcal{H}$ by the isogenies $p^{r_{i}}$ of the subquotients $\mathcal{H}^{i}$ is a filtered Barsotti-Tate group (with additional structures) which is split over $Z_{0}$. This defines a morphism $\delta: Z \rightarrow \mathcal{Y}$, and it is easy to see that it extends uniquely to a $J\left(\mathbb{Q}_{p}\right)$-equivariant morphism $\delta: \mathcal{F} \rightarrow \mathcal{Y}$. Moreover, it is also obvious from the definition that $\delta$ commutes with the descent data over $E$.

Finally, it is not hard to see that the diagram in the statement commutes. We verify the statement in the (EL) case, for $t=2$. (Similarly to Proposition 5.3, one can reduce the proof to this case.)

Let $\mathcal{H}$ be the universal Barsotti-Tate group defined over $\mathcal{F}, \alpha U^{\prime}$ its level structure over $\mathcal{F}^{U^{\prime}}$, and for simplicity write $r=r_{t}$. Then, $\alpha U^{\prime}$ gives rise to an isomorphism $\alpha: p^{-r} \Lambda / \Lambda \rightarrow$ $\mathcal{H}_{\eta}\left[p^{r}\right]$, compatible with the induced filtrations $\left(U^{\prime} \subseteq U_{r}\right)$. We define $\mathcal{K}=\alpha\left(\gamma^{-1} \Lambda / \Lambda\right) \subset$ $\mathcal{H}_{\eta}$. It follows from the definition of $\gamma$ that the natural projection $\mathcal{H}_{\eta} \rightarrow \mathcal{H}_{\eta}^{2}$ induces an isomorphism $\mathcal{K} \simeq \mathcal{H}_{\eta}^{2}\left[p^{r}\right]$ and that $\gamma^{*} \mathcal{H}_{\eta}=\mathcal{H}_{\eta} / \mathcal{K}$.

On the other hand, $\delta^{*}\left(\gamma^{*} \mathcal{H}_{\eta}\right)$ is by definition the pullback of $\gamma^{*} \mathcal{H}_{\eta}$ under multiplication by $p^{r}$ on $\mathcal{H}_{\eta}^{2}$. Thus, by construction, there is a natural quasi-isogeny

$$
\Phi: \delta^{*}\left(\gamma^{*} \mathcal{H}_{\eta}\right) \rightarrow \gamma^{*} \mathcal{H}_{\eta} \rightarrow \mathcal{H}_{\eta}
$$

Finally, we remark that $\Phi$ induces the identity on each subquotient of the filtrations and it is therefore an isomorphism.

Proposition 7.3. - The morphism $\Xi_{\eta}^{\mathcal{Y}}: \mathcal{Y}_{\eta} \rightarrow \mathcal{P}_{\eta}$ is smooth. Moreover, the associated map on the cohomology

$$
\left(\Xi_{\eta}^{\mathcal{Y}}\right)^{* \vee}: R \Gamma_{c}\left(\mathcal{Y}_{\eta} \times_{E_{0}} \hat{\bar{E}}_{0}, \mathbb{Q}_{l}\right) \rightarrow R \Gamma_{c}\left(\mathcal{P}_{\eta} \times_{E_{0}} \hat{\bar{E}}_{0}, \mathbb{Q}_{l}(-d)\right)[-2 d]
$$

is a quasi-isomorphism $\left(d=D_{\mathcal{F}}-D_{\mathcal{P}}\right)$.

Proof. - We work locally on $\mathcal{P}_{\eta}$, and in particular we consider a cover of opens of the form $S_{\eta}=\operatorname{Spm}\left(B \otimes E_{0}\right)$, where $S=\operatorname{SpfB}$ is a $p$-adic formal scheme equipped with a morphism $f: S \rightarrow \mathcal{P}$ (see [31], Section 5.5, p. 231).

Let $M^{i} \rightarrow \mathcal{L}^{i}$ denote the filtered Dieudonné crystals associated to the Barsotti-Tate groups $f^{*} \mathcal{H}^{i} / S$, and $\mathcal{E}^{i}=\operatorname{ker}\left(M^{i} \rightarrow \mathcal{L}^{i}\right)$, for all $i=1, \ldots, t$. We write $M=\oplus_{i} M^{i}$. Then, $\mathcal{Y}_{\mid S} \rightarrow S$ parameterizes all locally free quotients $\pi: M \rightarrow \mathcal{L}$ compatible with filtrations on the subquotients $M^{i} \rightarrow \mathcal{L}^{i}$ such that $\mathcal{L}_{\mid S_{0}}=\oplus_{i} \mathcal{L}_{\mid S_{0}}^{i}$.

Thus, we can identify

$$
\mathcal{Y}_{\mid S}=\underline{\operatorname{Hom}}\left(\mathcal{E}^{t}, p \mathcal{L}_{t-1}\right) \times \cdots \times \underline{\operatorname{Hom}}\left(\mathcal{E}^{2}, p \mathcal{L}_{1}\right),
$$

for $\mathcal{L}_{j}$ the locally free quotients of $M_{j}=\oplus_{1 \leq i \leq j} M^{i}$ defined as follows, for all $j=1, \ldots, t$. For $j=1$ we set $\mathcal{L}_{1}=\mathcal{L}^{1}$. For $j>1$ we define $\mathcal{L}_{j}$ by induction, as the universal extension of $\mathcal{L}^{j}$ by $\mathcal{L}_{j-1}$ over $\underline{\operatorname{Hom}}\left(\mathcal{E}^{j}, p \mathcal{L}_{j-1}\right)$.

In particular, locally on $\mathcal{P}_{\eta}, \mathcal{Y}_{\eta}$ is an open disk of relative dimension $d$, and thus $\left(\Xi_{\eta}^{\mathcal{Y}}\right)^{* \vee}$ is an isomorphism. 
COROllary 7.4. - The morphism

$$
\left(\Xi_{\eta}^{*}\right)^{\vee}: R \Gamma_{c}\left(\mathcal{F}_{\eta} \times_{E_{0}} \hat{\bar{E}}_{0}, \mathbb{Q}_{l}\right) \rightarrow R \Gamma_{c}\left(\mathcal{P}_{\eta} \times_{E_{0}} \hat{\bar{E}}_{0}, \mathbb{Q}_{l}(-d)\right)[-2 d]
$$

is a quasi-isomorphism.

Proof. - It follows from Proposition 7.3 and the equality $\left(\Xi_{\eta}^{\mathcal{Y}}\right)^{* \vee}=\left(\Xi_{\eta}^{*}\right)^{\vee} \circ\left(j^{*}\right)^{\vee}$ that it suffices to show that

$$
\left(j^{*}\right)^{\vee}=j_{!}: H_{c}^{i}\left(\mathcal{Y}_{\eta} \times_{E_{0}} \hat{\bar{E}}_{0}, \mathbb{Q}_{l}\right) \rightarrow H_{c}^{i}\left(\mathcal{F}_{\eta} \times_{E_{0}} \hat{\bar{E}}_{0}, \mathbb{Q}_{l}\right)
$$

is an isomorphism, for all $i \geq 0$. In fact, it suffices to show it is surjective since injectivity also follows from the previous result and the above equality.

The surjectivity of $j$ ! follows from the commutativity of the diagram in Proposition 7.2, i.e. from the equality $\left(f^{U}\right) !=j_{!} \circ \delta_{!} \circ \gamma_{!}$.

7.2. - We proceed to prove the analogue of Corollary 7.4 in the case of smaller levels $U \subseteq U_{0}$ (e.g. $U=U_{m}$, for $m \geq 1$ ). We remark that the above argument does not extend directly to the general case. In fact, as $U \subseteq U_{0}$ varies, the fibers of the morphism

$$
\Xi_{\mid \mathcal{Y} U}^{U}: \mathcal{Y}^{U}=\mathcal{F}^{U} \times \mathcal{F} \mathcal{Y} \rightarrow \mathcal{P}^{U}
$$

change.

Proposition 7.5. - For any positive integer $m$, there exists a formal scheme $\mathcal{Y}_{m}$ over $\mathcal{Y}$ satisfying the following properties.

1. Let $\mathcal{H}$ denote the universal Barsotti-Tate group over $\mathcal{Y}$. For any morphism of formal schemes $f: S \rightarrow \mathcal{Y}$, the $p^{m}$-torsion subgroup $f^{*} \mathcal{H}\left[p^{m}\right]$ is split if and only if $f$ factors via $j_{m}: \mathcal{Y}_{m} \rightarrow \mathcal{Y}$.

2. Let $\Xi^{\mathcal{Y}_{m}}: \mathcal{Y}_{m} \rightarrow \mathcal{P}$ denote the morphism $\Xi^{\mathcal{Y}} \circ j_{m}$. As a formal $\mathcal{P}$-scheme, $\mathcal{Y}_{m}$ is isomorphic to $\mathcal{Y}\left(\Xi^{\mathcal{Y}}: \mathcal{Y} \rightarrow \mathcal{P}\right)$.

3. The morphism $\Xi^{\mathcal{Y}_{m}}$ induces a quasi-isomorphism (for $d=D_{\mathcal{F}}-D_{\mathcal{P}}$ )

$$
\left(\Xi_{\eta}^{\mathcal{Y}_{m}}\right)^{* \vee}: R \Gamma_{c}\left(\mathcal{Y}_{m, \eta} \times_{E_{0}} \hat{\bar{E}}_{0}, \mathbb{Q}_{l}\right) \rightarrow R \Gamma_{c}\left(\mathcal{P}_{\eta} \times_{E_{0}} \hat{\bar{E}}_{0}, \mathbb{Q}_{l}(-d)\right)[-2 d] .
$$

Proof. - We construct $\mathcal{Y}_{m}$ by induction on $t$ the length of the filtration, as follows. For $t=1, \mathcal{Y}_{m}=\mathcal{Y}$. For $t>1$, let $\mathcal{Y}^{\prime} \subset \mathcal{Y}$ be the locus where $\mathcal{H}_{t-1}\left[p^{m}\right]$ is split, and consider the formal scheme over $\mathcal{Y}^{\prime}$ classifying finite flat subgroups $\mathcal{K}$ of $\mathcal{H}\left[p^{m}\right]$, of rank $p^{m h_{t}}$, which are stable under the action of $\mathcal{O}_{B}$. Inside it, there is the open subspace $W$ where the morphism $\mathcal{K} \subset \mathcal{H}\left[p^{m}\right] \rightarrow \mathcal{H}^{t}\left[p^{m}\right]$ is an isomorphism. Finally, we consider the closed locus $Z_{0}$ of $W_{0}$, $W_{0} \rightarrow Y_{0}$, where the two subgroups of $\mathcal{H}_{W_{0}}=\left(\mathcal{H}_{t-1} \oplus \mathcal{H}^{t}\right)_{W_{0}}, \mathcal{K}_{W_{0}}$ and $\mathcal{H}^{t}\left[p^{m}\right]_{W_{0}}$, coincide. We define $\mathcal{Y}_{m}$ as the formal completion of $W$ along $Z_{0}$.

Given the construction of $\mathcal{Y}_{m}$, Part 2 in the statement can be proved by induction on the length of the filtration, $t$. For simplicity, we prove this result in the case $t=2$. Let $\mathcal{H}$, $0 \rightarrow \mathcal{H}_{1} \rightarrow \mathcal{H} \rightarrow \mathcal{H}^{2} \rightarrow 0$, (resp. $\mathcal{H}^{\prime}, 0 \rightarrow \mathcal{H}_{1}^{\prime} \rightarrow \mathcal{H}^{\prime} \rightarrow \mathcal{H}^{\prime 2} \rightarrow 0$ ) denote the universal Barsotti-Tate group over $\mathcal{Y}$ (resp. over $\mathcal{Y}_{m}$ ). We write $\left[p^{m}\right]_{\mathcal{H}^{2}}$ for the multiplication by $p^{m}$ on $\mathcal{H}^{2}$. Then, the pullback of $\mathcal{H}$ under $\left[p^{m}\right]_{\mathcal{H}^{2}}$ is a Barsotti-Tate group with additional structures over $\mathcal{Y}$, with split $p^{m}$-torsion subgroup. Thus, associated with it there is a unique morphism $\phi: \mathcal{Y} \rightarrow \mathcal{Y}_{m}$. It follows from the definition that $\phi$ commutes with the structural morphisms of formal $\mathcal{P}$-schemes. On the other hand, let $\mathcal{K} \subset \mathcal{H}^{\prime}\left[p^{m}\right]$ denote the finite flat 
subgroup corresponding to the splitting (as defined above). The quotient $\mathcal{H}^{\prime} / \mathcal{K}$ is a BarsottiTate group over $\mathcal{Y}_{m}$ which inherits the additional structures of $\mathcal{H}^{\prime}$. Thus, associated with it there is a unique morphism $\psi: \mathcal{Y}_{m} \rightarrow \mathcal{Y}$, and $\psi$ commutes with the structural morphisms of formal $\mathcal{P}$-schemes. It is easy to check that these two morphisms are inverse of each other.

Part 3 is an immediate consequence of Part 2 and Proposition 7.3.

For each $m \geq 1$, we consider the level $U=U_{m} \subset P\left(\mathbb{Q}_{p}\right)$. We define two distinct covers of level $U$ of the rigid analytic fiber $\mathcal{Y}_{m, \eta}$ of $\mathcal{Y}_{m}$ as follows.

First, we regard the formal scheme $\mathcal{Y}_{m}$ as a $\mathcal{F}$-scheme via the morphism $j_{\mathcal{Y}_{m}}=j \circ j_{m}$. We define $\mathcal{Y}_{m}^{U} \rightarrow \mathcal{Y}_{m, \eta}$ as the pullback of the cover $\mathcal{F}^{U} \rightarrow \mathcal{F}_{\eta}$, i.e.

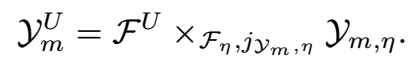

We write $j_{\mathcal{Y}_{m}^{U}}=\mathbb{I}_{\mathcal{F} U} \times j_{\mathcal{Y}_{m}}: \mathcal{Y}_{m}^{U} \rightarrow \mathcal{F}^{U}$ for the natural projection.

Secondly, we regard $\mathcal{Y}_{m}$ as a $\mathcal{P}$-scheme via the morphism $\Xi^{\mathcal{Y}_{m}}=\Xi^{\mathcal{Y}} \circ j_{m}$. We define $\mathcal{Y}_{m}^{(U)} \rightarrow \mathcal{Y}_{m, \eta}$ as the pullback of the cover $\mathcal{P}^{U} \rightarrow \mathcal{P}_{\eta}$ (here, with abuse of notations, we write $\mathcal{P}^{U}$ in place of $\left.\mathcal{P}^{U \cap L\left(\mathbb{Q}_{p}\right)}\right)$, i.e.

$$
\mathcal{Y}_{m}^{(U)}=\mathcal{P}^{U} \times_{\mathcal{P}_{\eta}, \Xi_{\eta}^{\mathcal{y}_{m}}} \mathcal{Y}_{m, \eta}
$$

We write $\Xi_{\mathcal{P} U}^{\mathcal{Y}_{m}}=\mathbb{I}_{\mathcal{P}_{U}} \times \Xi^{\mathcal{Y}_{m}}: \mathcal{Y}_{m}^{(U)} \rightarrow \mathcal{P}^{U}$ for the natural projection.

It follows from Part 3 of the above proposition that $\Xi_{\mathcal{P} U}^{\mathcal{Y}_{m}}$ induces a quasi-isomorphism $\left(\Xi_{\mathcal{P}^{U}}^{\mathcal{Y}_{m}}\right)_{\eta}^{* \vee}: R \Gamma_{c}\left(\mathcal{Y}_{m, \eta}^{(U)} \times_{E_{0}} \hat{\bar{E}}_{0}, \mathbb{Q}_{l}\right) \rightarrow R \Gamma_{c}\left(\mathcal{P}_{\eta}^{U} \times_{E_{0}} \hat{\bar{E}}_{0}, \mathbb{Q}_{l}(-d)\right)[-2 d], \quad d=D_{\mathcal{F}}-D_{\mathcal{P}}$.

Lemma 7.6. - 1 . There exists a morphism $\pi^{U}: \mathcal{Y}_{m}^{U} \rightarrow \mathcal{Y}_{m}^{(U)}$ satisfying the condition $\Xi_{\mathcal{P}^{U}}^{\mathcal{Y}_{m}} \circ \pi^{U}=\Xi^{U} \circ j_{\mathcal{Y}_{m}^{U}}$.

2. There exists a morphism $s^{U}: \mathcal{Y}_{m}^{(U)} \rightarrow \mathcal{Y}_{m}^{U}$ such that $\pi^{U} \circ s^{U}=\mathbb{I}_{\mathcal{Y}^{(U)}}$.

Proof. - The first part is an immediate consequence of the definitions. For the second part, we observe that the existence of a splitting $\mathcal{H}\left[p^{m}\right] \simeq \oplus_{i} \mathcal{H}^{i}\left[p^{m}\right]$ over $\mathcal{Y}_{m}$ allows us to define a structure of level $U$ on $\mathcal{H}$ starting from the level structures on the subquotients $\mathcal{H}^{i}$. The existence of such a level structure is equivalent to the datum of a section $s^{U}: \mathcal{Y}_{m}^{(U)} \rightarrow \mathcal{Y}_{m}^{U}$ of $\pi^{U}$.

The following proposition is an analogue of Proposition 7.2.

Proposition 7.7. - For a given integer $m \geq 0$, let $U=U_{m}$.

Then, there exist some integers $0=r_{1} \leq r_{2} \leq \cdots \leq r_{t}$ (in the (PEL) case satisfying the conditions $r_{i}+r_{j}=r_{t}$ for all $i, j$ with $i+j=t+1$ ) and a morphism $\delta_{m, r}^{U}: \mathcal{F}^{U} \rightarrow \mathcal{Y}_{m}^{U}$ such that

1. $\delta_{m, r}$ is equivariant for the action of $J\left(\mathbb{Q}_{p}\right)$ and the descent data,

2. $\delta_{m, r}$ factors through the map $s^{U}: \mathcal{Y}_{m}^{(U)} \rightarrow \mathcal{Y}_{m}^{U}$,

3. for a level $U^{\prime} \subseteq U$ sufficiently small, we have the equality

$$
\begin{gathered}
f_{U}^{U^{\prime}}=j_{\mathcal{Y}_{m}^{U}} \circ \delta_{m, r}^{U} \circ \gamma_{m, r}, \\
\text { where } \gamma_{m, r}=\left(1, p^{-m-r_{2}}, p^{-2 m-r_{3}}, \ldots, p^{(1-t) m-r_{t}}\right) \in P\left(\mathbb{Q}_{p}\right) .
\end{gathered}
$$


Proof. - It follows from Proposition 5.4 that the map that to a filtered Barsotti-Tate group with additional structure $G / S$ associates its pullback by the isogenies $p^{(i-1) m}$ on the subquotients $G^{i} / S$ defines a $J\left(\mathbb{Q}_{p}\right) \times W_{E}$-equivariant morphism

$$
\delta_{m}: \mathcal{Y} \rightarrow \mathcal{Y}_{m}
$$

such that $f_{\mid \mathcal{Y}}^{U^{\prime}}=j_{m} \circ \delta_{m} \circ \gamma_{m}$, for $\gamma_{m}=\left(1, p^{-m}, p^{-2 m}, \ldots, p^{(1-t) m}\right) \in P\left(\mathbb{Q}_{p}\right)$ and $U^{\prime}$ a sufficiently small level.

By composing $\delta_{m}$ with the morphism $\delta$ defined in Proposition 7.2, we obtain a morphism

$$
\delta_{m, r}: \mathcal{F} \rightarrow \mathcal{Y}_{m}
$$

satisfying the equation $f^{U^{\prime}}=j \mathcal{Y}_{m} \circ \delta_{m, r} \circ \gamma_{m, r}$, for $\gamma_{m, r}=\gamma_{m} \circ \gamma \quad$ and $\gamma=\left(1, p^{-r_{2}}, \ldots, p^{-r_{t}}\right) \in P\left(\mathbb{Q}_{p}\right)$ and $U^{\prime}$ sufficiently small, as in Proposition 7.2.

We claim that for $U=U_{m}$ the morphism $\delta_{m, r}: \mathcal{F} \rightarrow \mathcal{Y}_{m}$ lifts uniquely to a morphism $\delta_{m, r}^{U}: \mathcal{F}^{U} \rightarrow \mathcal{Y}_{m}^{U}$. In fact, to a level structure on $\mathcal{H}_{\eta}$ one can associate the level structure on $\delta_{m, r}^{*} \mathcal{H}_{\eta}$ defined by the induced level structure on the subquotients $\mathcal{H}_{\eta}^{i}$ together with the datum of a splitting of $\mathcal{H}_{\eta}\left[p^{m}\right]$. Moreover, $\delta_{m, r}^{U}$ factors via the section $s^{U}: \mathcal{Y}_{m}^{(U)} \rightarrow \mathcal{Y}_{m}^{U}$.

Finally, for a sufficiently small level $U^{\prime} \subseteq U=U_{m}$, we obtain the equality $f_{U}^{U^{\prime}}=j_{\mathcal{Y}_{m}^{U}} \circ \delta_{m, r}^{U} \circ \gamma_{m, r}$.

By arguing as in Corollary 7.4, Proposition 7.7 implies the following result.

Proposition 7.8. - The morphism $\Xi^{U}: \mathcal{F}^{U} \rightarrow \mathcal{P}^{U}$ induces is a quasi-isomorphism

$$
\left(\Xi_{\eta}^{U}\right)^{* \vee}: R \Gamma_{c}\left(\mathcal{F}_{\eta}^{U} \times_{E_{0}} \hat{\bar{E}}_{0}, \mathbb{Q}_{l}\right) \rightarrow R \Gamma_{c}\left(\mathcal{P}_{\eta}^{U} \times_{E_{0}} \hat{\bar{E}}_{0}, \mathbb{Q}_{l}(-d)\right)[-2 d], \quad d=D_{\mathcal{F}}-D_{\mathcal{P}},
$$

equivariant for the action of $J\left(\mathbb{Q}_{p}\right)$ and $W_{E}$.

As the level $U$ varies, the above isomorphisms form a projective system, compatible with the action of $J\left(\mathbb{Q}_{p}\right), W_{E}$ and $P\left(\mathbb{Q}_{p}\right)$. This observation suffices to deduce Theorem 7.1 from the above results.

7.3. - We conclude this section by observing that for a pair $(b, \mu)$ of $(\mathrm{HN})$ type and $M \supseteq M_{b}$, the geometry of the corresponding space $\mathcal{F}=\mathcal{F}_{P_{M}}$ is simpler than in the general case we just considered. In fact, under this hypothesis, Theorem 6.2 implies that the morphism $\Theta: \mathcal{P}_{0} \rightarrow \mathcal{F}_{0}$ is actually an isomorphism, i.e. that $\mathcal{Y}_{0}=\mathcal{F}_{0}$ and thus $\mathcal{Y}=\mathcal{F}$.

\section{Geometric realization of parabolic induction}

In this section we proceed to compare the projective system of moduli spaces $\mathcal{F}^{\infty}$, regarded as a tower over $\mathcal{F}^{U_{0}}$, with the pullback over $\mathcal{F}^{U_{0}}$ of the tower $\mathcal{M}^{\infty} / \mathcal{M}^{U_{0}}$. We denote the latter by $\mathcal{M}^{\infty} \times{ }_{\mathcal{M}^{U_{0}}} \mathcal{F}^{U_{0}}$. The first system classifies filtered Barsotti-Tate groups endowed with a level structure which preserves the filtration, while the second classifies filtered Barsotti-Tate groups endowed with any level structure (not necessarily preserving the filtration). 
8.1. - Similarly to the case of $\mathcal{M}^{\infty}$, when studying the tower $\mathcal{M}^{\infty} \times{ }_{\mathcal{M}^{U_{0}}} \mathcal{F}^{U_{0}}$, we are interested in studying the limit of the $l$-adic étale cohomology groups of the rigid analytic spaces $\mathcal{M}^{U} \times \mathcal{M}_{U_{0}} \mathcal{F}^{U_{0}}, U \subseteq U_{0}$, as representations of $G\left(\mathbb{Q}_{p}\right) \times W_{E}$ and $J\left(\mathbb{Q}_{p}\right)$.

More precisely, let $l$ be a prime number, $l \neq p$, and $\hat{\bar{E}_{0}}$ the completion of an algebraic closure of $E_{0}$. For any level $U \subseteq U_{0}$, we consider the cohomology groups

$$
\begin{aligned}
H^{i}\left(\mathcal{M}^{U} \times_{\mathcal{M}^{U_{0}}} \mathcal{F}^{U_{0}}\right) & =H_{c}^{i}\left(\left(\mathcal{M}^{U} \times_{\mathcal{M}^{U_{0}}} \mathcal{F}^{U_{0}}\right) \times_{E_{0}} \hat{\bar{E}_{0}}, \mathbb{Q}_{l}\left(D_{\mathcal{F}}\right)\right) \\
& =\underline{\lim } \mathcal{V} H_{c}^{i}\left(\mathcal{V} \times_{E_{0}} \hat{\bar{E}_{0}}, \mathbb{Q}_{l}\left(D_{\mathcal{F}}\right)\right)
\end{aligned}
$$

where $\mathcal{V}$ varies among the open subspaces of $\mathcal{M}^{U} \times{ }_{\mathcal{M}^{U_{0}}} \mathcal{F}^{U_{0}}$ of finite type, and $D_{\mathcal{F}}=$ $\operatorname{dim} \mathcal{F}^{U}=\operatorname{dim}\left(\mathcal{M}^{U} \times \mathcal{M}_{\mathcal{M}_{0}} \mathcal{F}^{U_{0}}\right)(i \in \mathbb{N})$. On them, there is a natural action of $J\left(\mathbb{Q}_{p}\right)$ and $W_{E}$ induced by the action of $J\left(\mathbb{Q}_{p}\right)$ and the descent data over $E$ on the spaces $\mathcal{M}^{U} \times{ }_{\mathcal{M}^{U_{0}}} \mathcal{F}^{U_{0}}$.

As the level $U$ varies, these cohomology groups form a projective limit which is naturally endowed with an action of $G\left(\mathbb{Q}_{p}\right)$ induced by the action of $G\left(\mathbb{Q}_{p}\right)$ on the tower $\mathcal{M}^{\infty} \times_{\mathcal{M}^{U_{0}}}$ $\mathcal{F}^{U_{0}}$.

Moreover, as in the case of the cohomology of $\mathcal{F}^{\infty}$, for any $l$-adic admissible representation $\rho$ of $J\left(\mathbb{Q}_{p}\right)$, the groups

$$
H^{i, j}\left(\mathcal{M}^{\infty} \times_{\mathcal{M}^{U_{0}}} \mathcal{F}^{U_{0}}\right)_{\rho}=\lim _{U} \operatorname{Ext}_{J\left(\mathbb{Q}_{p}\right)}^{j}\left(H^{i}\left(\mathcal{M}^{U} \times_{\mathcal{M}^{U_{0}}} \mathcal{F}^{U_{0}}\right), \rho\right)
$$

vanish for almost all $i, j \geq 0$, and the following equalities define a virtual representation of $G\left(\mathbb{Q}_{p}\right) \times W_{E}$

$$
H^{\bullet}\left(\mathcal{M}^{\infty} \times_{\mathcal{M}^{U_{0}}} \mathcal{F}^{U_{0}}\right)_{\rho}=\sum_{i, j \geq 0}(-1)^{i+j} H^{i, j}\left(\mathcal{M}^{\infty} \times{ }_{\mathcal{M}^{U_{0}}} \mathcal{F}^{U_{0}}\right)_{\rho}
$$

The goal of this section is to prove the following theorem.

THEOREM 8.1. - For any l-adic admissible representation $\rho$ of $J\left(\mathbb{Q}_{p}\right)$, we have an equality of virtual representations of $G\left(\mathbb{Q}_{p}\right) \times W_{E}$ :

$$
H^{\bullet}\left(\mathcal{M}^{\infty} \times \mathcal{M}_{U_{0}} \mathcal{F}^{U_{0}}\right)_{\rho}=\operatorname{Ind}_{P\left(\mathbb{Q}_{p}\right)}^{G\left(\mathbb{Q}_{p}\right)}\left(H^{\bullet}\left(\mathcal{F}^{\infty}\right)_{\rho}\right)
$$

Our argument follows [4] (see also [12], p. 115).

8.2. - We fix a level $U$. For convenience we choose $U$ of the form $U=U_{m}, m \geq 1$.

For any point $x$ of $\mathcal{M}^{U} \times \mathcal{M}^{U_{0}} \mathcal{F}^{U_{0}}$, we denote by $(G, \alpha U)$ the data of a filtered BarsottiTate group with level structure, corresponding to $x$. Then, the image under $\alpha$ of the filtration on $G$ uniquely determines a filtration $N(x) \bullet$ on $p^{-m} \Lambda / \Lambda$ by $\mathcal{O}_{B} / p^{m}$-submodules (with $\mathbb{Z} / p^{m} \mathbb{Z}$-free subquotients). In the (PEL) case, this filtration is symplectic, i.e. compatible with the alternating pairing on $p^{-m} \Lambda / \Lambda$. Moreover, the filtration $N(x) \bullet$ is obviously the reduction modulo $p^{m}$ of a (symplectic) $\mathcal{O}_{B}$-linear filtration of $\Lambda$, with $\mathbb{Z}_{p}$-free subquotients of $\operatorname{rank} h_{i}=h t\left(\Sigma^{i}\right), i=1, \ldots, t$.

For any (in the (PEL) case symplectic) $\mathcal{O}_{B}$-linear filtration $N_{\bullet}$ of $p^{-m} \Lambda / \Lambda$, with $\mathbb{Z} / p^{m} \mathbb{Z}$ free subquotients, we consider the subspace $\mathcal{F}_{N_{\bullet}}^{U}$ of $\mathcal{M}^{U} \times{ }_{\mathcal{M}^{U_{0}}} \mathcal{F}^{U_{0}}$ consisting of all points $x$ such that $N(x) \bullet=N_{\bullet}$. The subspaces $\mathcal{F}_{N_{\bullet}}^{U}$ are both open and closed, and therefore define a decomposition

$$
\mathcal{M}^{U} \times_{\mathcal{M}^{U_{0}}} \mathcal{F}^{U_{0}}=\coprod_{N_{\bullet} \subset p^{-m} \Lambda / \Lambda} \mathcal{F}_{N_{\bullet}}^{U}
$$


indexed by the reduction modulo $p^{m}$ of all (symplectic) $\mathcal{O}_{B}$-linear filtrations of $\Lambda$, with $\mathbb{Z}_{p}$-free subquotients of rank $h_{i}(i=1, \ldots, t)$. It is easy to see that the action of $G\left(\mathbb{Z}_{p}\right)$ on the moduli space $\mathcal{M}^{U} \times \mathcal{M}^{U_{0}} \mathcal{F}^{U_{0}}$ preserves the above decomposition, and that the stabilizer of the filtration $N_{\bullet}$ stabilizes the associated subspace $\mathcal{F}_{N_{\bullet}}^{U}$.

Furthermore, after the choice of a (symplectic) $\mathcal{O}_{B}$-linear automorphism of $\Lambda$, mapping $N_{\bullet}$ onto the canonical filtration $p^{-m} \Lambda_{\bullet} / \Lambda_{\bullet}$ of $p^{-m} \Lambda / \Lambda$, we can identify the subspace $\mathcal{F}_{N_{\bullet}}^{U}$ with the moduli space $\mathcal{F}^{U}=\mathcal{F}_{p^{-m} \Lambda_{\bullet} / \Lambda_{\bullet}}$ (and the action of the stabilizer of $N_{\bullet}$ on $\mathcal{F}_{N_{\bullet}}^{U}$ with the action of $P\left(\mathbb{Z}_{p}\right)$ on $\left.\mathcal{F}^{U}\right)$. Therefore, it is possible to rewrite the above decomposition as

$$
\mathcal{M}^{U} \times \mathcal{M}^{U_{0}} \mathcal{F}^{U_{0}}=\coprod_{P\left(\mathbb{Q}_{p}\right) \backslash G\left(\mathbb{Q}_{p}\right) / U} \mathcal{F}^{U},
$$

and compute the cohomology groups of $\mathcal{M}^{U} \times{ }_{\mathcal{M}^{U_{0}}} \mathcal{F}^{U_{0}}$, regarded as $l$-adic representations of $G\left(\mathbb{Q}_{p}\right) \times J\left(\mathbb{Q}_{p}\right) \times W_{E}$, as

$$
H_{c}^{i}\left(\left(\mathcal{M}^{U} \times_{\mathcal{M}^{U_{0}}} \mathcal{F}^{U_{0}}\right) \times_{E_{0}} \hat{\bar{E}}_{0}, \mathbb{Q}_{l}\right)=\oplus_{P\left(\mathbb{Q}_{p}\right) \backslash G\left(\mathbb{Q}_{p}\right) / U} H_{c}^{i}\left(\mathcal{F}^{U} \times_{E_{0}} \hat{\bar{E}}_{0}, \mathbb{Q}_{l}\right) \quad \text { for all } i \geq 0 .
$$

As we let the level $U$ vary, these decompositions are preserved under the natural projections and the group action, thus they imply Theorem 8.1.

\section{Forgetting the filtration: the general case}

We are now left with a last task: comparing the towers of moduli spaces $\mathcal{M}^{\infty}$ and $\mathcal{M}^{\infty} \times \mathcal{M}^{U_{0}} \mathcal{F}^{U_{0}}$.

For a general admissible pair $(b, \mu)$, the Barsotti-Tate groups classified by the RapoportZink spaces do not always admit a slope decomposition or filtration (nor coarser ones), and as a consequence of this fact our results are quite weak in this generality. On the other hand, for pairs $(b, \mu)$ of $(\mathrm{HN})$ type, our analysis leads to stronger results. We postpone the discussion of these favorable cases to Section 10 . In this section, we simply try to shed some light on the image of the morphism $j: \mathcal{F} \rightarrow \mathcal{M}$, in the case of a general admissible pair and for any choice of a Levi subgroup $M, L_{b} \subseteq M \subset G$.

9.1. - As in Section 7, let $\mathcal{M}_{0}=V(I)$ and $\mathcal{F}_{0}=V(J)$, for $I$ and $J$ ideals of definition of $\mathcal{M}$ and $\mathcal{F}$ respectively $\left(j^{*} I \subset J\right)$.

Let $Z_{0}$ be a closed $k$-subscheme of finite type such that $\mathcal{M}_{0}=\cup_{t \in J\left(\mathbb{Q}_{p}\right)} t Z_{0}$. We write $Z$ for the formal completion of $\mathcal{M}$ along $Z_{0}$. Thus $Z^{\text {rig }}=s p^{-1}\left(Z_{0}\right)$ (for $s p: \mathcal{M}^{\text {rig }} \rightarrow \mathcal{M}_{0}$ the specialization map) is an open subspace of $\mathcal{M}^{\text {rig }}$ satisfying $\mathcal{M}^{\text {rig }}=\cup_{t \in J\left(\mathbb{Q}_{p}\right)} t Z^{\text {rig }}$. We remark that it is possible to choose $Z_{0}$ such that it is stable under the action of a maximal compact $\Gamma \subset J\left(\mathbb{Q}_{p}\right)$, i.e. $\mathcal{M}_{0}=\cup_{t \in J\left(\mathbb{Q}_{p}\right) / \Gamma} t Z_{0}$. For such $Z_{0}$, we have that $Z^{\text {rig }}$ is also stable under the action of $\Gamma$ and $\mathcal{M}^{\text {rig }}=\cup_{t \in J\left(\mathbb{Q}_{p}\right) / \Gamma} t Z^{\text {rig }}$.

Let $\left\{V_{\alpha}\right\}_{\alpha}$ denote the locally closed stratification of $Z_{0}$ as in Proposition 5.1, and $\left\{\mathcal{V}_{\alpha}\right\}_{\alpha}$ be the locally closed stratification of $Z$ obtained as the formal completion of $\mathcal{M}$ along the strata $V_{\alpha}$ (for all $\alpha, \mathcal{V}_{\alpha}^{\text {rig }}=s p^{-1}\left(V_{\alpha}\right)$ ). We remark that, when $Z_{0}$ is stable under the action of $\Gamma$, the above strata are also stable under this action. 
Lemma 9.1. - For each $\alpha$, we write $\mathcal{U}_{\alpha}=j^{-1} \mathcal{V}_{\alpha}$ and $j_{\alpha}: \mathcal{U}_{\alpha} \rightarrow \mathcal{V}_{\alpha}$ for the restriction of $j$ to $\mathcal{U}_{\alpha}$.

Then, the $j_{\alpha}$ are closed immersions and their reduced fibers $j_{U_{\alpha}}^{\text {red }}: \mathcal{U}_{\alpha}^{\text {red }} \rightarrow \mathcal{V}_{\alpha}^{\text {red }}$ isomorphisms, for all $\alpha$.

Proof. - We recall that, by construction, the restriction of the universal Barsotti-Tate group $\mathcal{G}$ to each $V_{\alpha}=\left(\mathcal{V}_{\alpha}\right)_{0}$ admits a (unique) filtration. In particular, the morphism between reduced fibers $j_{\alpha}^{\text {red }}: \mathcal{U}_{\alpha}^{\text {red }} \rightarrow \mathcal{V}_{\alpha}^{\text {red }}$ admits a section, which is in fact an inverse because of the uniqueness of the filtration.

Moreover, it follows from Proposition 5.8 that the $j_{\alpha}$ are closed immersions, for all $\alpha$.

The main result of this section is the following theorem.

THEOREM 9.2. - There exists a closed formal subscheme, formally locally of finite type,

$$
i: \mathcal{X}=\mathcal{X}_{P} \hookrightarrow \mathcal{M}
$$

defined over $\mathcal{O}_{E_{0}}$, which is invariant under the action of $J\left(\mathbb{Q}_{p}\right)$ and the descent data to $E$, and satisfies the following conditions:

1. its reduced fiber $\mathcal{X}^{\mathrm{red}}=\mathcal{M}^{\mathrm{red}}$;

2. its rigid analytic fiber $\mathcal{X}^{\text {rig }}$ is smooth of dimension $D_{\mathcal{X}}=D_{\mathcal{F}}$;

3. the morphism $j: \mathcal{F} \rightarrow \mathcal{M}$ factors through the inclusion $i: \mathcal{X} \hookrightarrow \mathcal{M}$, and moreover, for a stratification $\left\{\mathcal{V}_{\alpha}\right\}$ of $\mathcal{M}$ defined as in 9.1, the restrictions of $j$ to the strata

$$
j_{\alpha}: \mathcal{U}_{\alpha}=j^{-1}\left(\mathcal{V}_{\alpha}\right) \rightarrow \mathcal{X} \cap \mathcal{V}_{\alpha}
$$

are isomorphisms.

In particular, $\mathcal{X}$ is the image of $j$.

9.2. - We assume for the moment that such an $\mathcal{X}$ exists, and write $\mathcal{X}^{\infty}$ for the projective system $\left\{\mathcal{M}^{U} \times_{\mathcal{M}^{U_{0}}} \mathcal{X}^{\text {rig }}\right\}_{U \subseteq U_{0}}$, obtained as the pullback of $\mathcal{M}^{\infty}$ via the inclusion $i: \mathcal{X} \rightarrow \mathcal{M}$. Then $\mathcal{X}^{\infty}$ is naturally endowed with an action of $J\left(\mathbb{Q}_{p}\right), W_{E}$ and $G\left(\mathbb{Q}_{p}\right)$.

Similarly to the case of the projective system $\mathcal{M}^{\infty} \times_{\mathcal{M}^{U_{0}}} \mathcal{X}^{\text {rig }}$, for $l$ a prime number, $l \neq p$, and for any $l$-adic admissible representation $\rho$ of $J\left(\mathbb{Q}_{p}\right)$, we define a virtual representation of $G\left(\mathbb{Q}_{p}\right) \times W_{E}$

$$
H^{\bullet}\left(\mathcal{X}^{\infty}\right)_{\rho}=\sum_{i, j \geq 0}(-1)^{i+j} H^{i, j}\left(\mathcal{X}^{\infty}\right)_{\rho}
$$

where, for all $i, j \geq 0$,

$$
H^{i, j}\left(\mathcal{X}^{\infty}\right)_{\rho}=\underline{\lim }_{U} \operatorname{Ext}_{J\left(\mathbb{Q}_{p}\right)}^{j}\left(H^{i}\left(\mathcal{X}^{U}\right), \rho\right)
$$

are some admissible representations of $G\left(\mathbb{Q}_{p}\right) \times W_{E}$ (almost all vanishing), and

$$
H^{i}\left(\mathcal{X}^{U}\right)=H_{c}^{i}\left(\mathcal{X}^{U} \times_{E_{0}} \hat{\overline{E_{0}}}, \mathbb{Q}_{l}\left(D_{\mathcal{X}}\right)\right)
$$

the (twisted) $l$-adic cohomology with compact support of the rigid analytic space $\mathcal{X}^{U}$.

Then, the above theorem implies the following result. 
THEOREM 9.3. - For any l-adic admissible representation $\rho$ of $J\left(\mathbb{Q}_{p}\right)$, we have an equality of virtual representations of $G\left(\mathbb{Q}_{p}\right) \times W_{E}$ :

$$
H^{\bullet}\left(\mathcal{X}^{\infty}\right)_{\rho}=H^{\bullet}\left(\mathcal{M}^{\infty} \times \mathcal{M}^{U_{0}} \mathcal{F}^{U_{0}}\right)_{\rho},
$$

and moreover

$$
H^{\bullet}\left(\mathcal{X}^{\infty}\right)_{\rho}^{U_{0}}=H^{\bullet}\left(\mathcal{M}^{\infty}\right)_{\rho}^{U_{0}}
$$

Proof. - Let $j^{\prime}: \mathcal{F} \rightarrow \mathcal{X}$ be the morphism corresponding to the factorization of $j: \mathcal{F} \rightarrow \mathcal{M}$ via $i: \mathcal{X} \rightarrow \mathcal{M}$.

Let $Z^{\text {rig }} \subset \mathcal{M}^{\text {rig }}$ be an open subspace, stable under the action of a maximal subgroup $\Gamma$ of $J\left(\mathbb{Q}_{p}\right)$, satisfying the condition $\mathcal{M}^{\text {rig }}=\cup_{t \in J\left(\mathbb{Q}_{p}\right) / \Gamma} Z^{\text {rig }}$, as in Section 9.1. For any level $U \subseteq U_{0}$, let $Z^{U}=\mathcal{M}^{U} \times Z^{\mathrm{rig}}$.

It is possible to compute the cohomology of the tower of Rapoport-Zink spaces in terms of the cohomology of certain subspaces, associated with the $Z^{U}$ 's (see [22], Section 8.2, and [8], Section 4.3). More precisely, the following holds.

For $U \subseteq U_{0}$, there exists a spectral sequence of $l$-adic representations of $J\left(\mathbb{Q}_{p}\right) \times W_{E}$

$$
E_{1}^{p, q}=\oplus_{\epsilon \in \Gamma \backslash\left(J\left(\mathbb{Q}_{p}\right) / \Gamma\right)_{\neq}^{p}} c-\operatorname{Ind}_{\Gamma_{\epsilon}}^{J\left(\mathbb{Q}_{p}\right)} H_{c}^{q}\left(Z_{\epsilon}^{U}, \mathbb{Q}_{l}\right) \Rightarrow H_{c}^{p+q}\left(\mathcal{M}^{U}, \mathbb{Q}_{l}\right),
$$

where

1. for $p \geq 1,\left(J\left(\mathbb{Q}_{p}\right) / \Gamma\right)_{\neq}^{p}$ is the subset of $\left(J\left(\mathbb{Q}_{p}\right) / \Gamma\right)^{p}$ consisting of all the elements $\epsilon=$ $\left(\epsilon_{1}, \ldots, \epsilon_{p}\right)$ such that $\epsilon_{i} \Gamma \neq \epsilon_{j} \Gamma \neq \Gamma$, for all $i \neq j$, and $\Gamma \backslash\left(J\left(\mathbb{Q}_{p}\right) / \Gamma\right)_{\neq}^{p}$ is its quotient by the action of $\Gamma$ via multiplication on the left (for $p=0, \Gamma \backslash\left(J\left(\mathbb{Q}_{p}\right) / \Gamma\right)_{\neq}^{p}=\{1\}$ );

2. for $p \geq 1$ and $\epsilon \in \Gamma \backslash\left(J\left(\mathbb{Q}_{p}\right) / \Gamma\right)_{\neq}^{p}, \Gamma_{\epsilon}=\Gamma \cap \epsilon_{1} \Gamma \epsilon_{1}^{-1} \cap \cdots \cap \epsilon_{p} \Gamma \epsilon_{p}^{-1}$ is a compact subgroup of $J\left(\mathbb{Q}_{p}\right)$ (for $p=0, \Gamma_{\{1\}}=\Gamma$ );

3. for $p \geq 1$ and $\epsilon \in \Gamma \backslash\left(J\left(\mathbb{Q}_{p}\right) / \Gamma\right)_{\neq}^{p}, Z_{\epsilon}^{U}=Z^{U} \cap \epsilon_{1} Z^{U} \cap \cdots \cap \epsilon_{p} Z^{U}$ is an open subset of $\mathcal{M}^{U}$, stable under the action of $\Gamma_{\epsilon}$ (for $p=0, Z_{\{1\}}^{U}=Z^{U}$ ).

Moreover, for any $p \geq 1$, the set $\left\{\epsilon \in \Gamma \backslash\left(J\left(\mathbb{Q}_{p}\right) / \Gamma\right)_{\neq}^{p} \mid Z_{\epsilon}^{U} \neq \varnothing\right\}$ is finite, and it is empty for $p$ sufficiently large.

In particular, for any $l$-adic admissible representation $\rho$ of $J\left(\mathbb{Q}_{p}\right)$, we have an equality of virtual admissible representations of $G\left(\mathbb{Q}_{p}\right) \times W_{E}$

$$
H^{\bullet}\left(\mathcal{M}^{\infty}\right)_{\rho}=\sum_{p \geq 1}(-1)^{p} \sum_{\epsilon \in \Gamma \backslash\left(J\left(\mathbb{Q}_{p}\right) / \Gamma\right)_{\neq}^{p}}\left(c-\operatorname{Ind}_{\Gamma_{\epsilon}}^{J\left(\mathbb{Q}_{p}\right)} H^{\bullet}\left(Z_{\epsilon}^{\infty}\right)\right)_{\rho}
$$

where, for each $\epsilon,\left(c-\operatorname{Ind}_{\Gamma_{\epsilon}}^{J\left(\mathbb{Q}_{p}\right)} H^{\bullet}\left(Z_{\epsilon}^{\infty}\right)\right)_{\rho}$ denotes the virtual representation

$$
\sum_{i, j}(-1)^{i+j} \lim _{\longrightarrow \subseteq U_{0}} \operatorname{Ext}_{J\left(\mathbb{Q}_{p}\right)}^{i}\left(c-\operatorname{Ind}_{\Gamma_{\epsilon}}^{J\left(\mathbb{Q}_{p}\right)} H_{c}^{j}\left(Z_{\epsilon}^{U} \times_{E_{0}} \hat{\bar{E}}_{0}, \mathbb{Q}_{l}\left(D_{\mathcal{M}}\right)\right), \rho\right) .
$$

Similar computations can be carried out for the cohomology of the projective systems $\mathcal{X}^{\infty}$ and $\mathcal{M}^{\infty} \times_{\mathcal{M}^{U_{0}}} \mathcal{F}^{U_{0}}$.

In fact, let us consider $C=Z \cap \mathcal{X}$. Then $C^{\text {rig }}$ is an open subspace of $\mathcal{X}^{\text {rig }}$, which is stable under the action of $\Gamma \subset J\left(\mathbb{Q}_{p}\right)$ and satisfies the condition $\mathcal{X}^{\text {rig }}=\cup_{t \in J\left(\mathbb{Q}_{p}\right)} C^{\text {rig. }}$. For any level $U \subseteq U_{0}$, we write $C^{U}=\mathcal{M}^{U} \times C^{\text {rig }} \subset \mathcal{X}^{U}$ (and $C_{\epsilon}^{U}=Z_{\epsilon}^{U} \cap \mathcal{X}^{U}$, for all $\epsilon$ as above). 
Then, one can apply the same arguments used in [22] and [8], to deduce that, for any level $U$, there exists a spectral sequence analogous to the one above, computing the $l$-adic cohomology of the rigid analytic space $\mathcal{X}^{U}$ in terms of the cohomology of the subspaces $C_{\epsilon}^{U}$. In particular, there is an equality of virtual admissible representations of $G\left(\mathbb{Q}_{p}\right) \times W_{E}$

$$
H^{\bullet}\left(\mathcal{X}^{\infty}\right)_{\rho}=\sum_{p \geq 1}(-1)^{p} \sum_{\epsilon \in \Gamma \backslash\left(J\left(\mathbb{Q}_{p}\right) / \Gamma\right)_{\neq}^{p}}\left(c-\operatorname{Ind}_{\Gamma_{\epsilon}}^{J\left(\mathbb{Q}_{p}\right)} H^{\bullet}\left(C_{\epsilon}^{\infty}\right)\right)_{\rho}
$$

where, for each $\epsilon,\left(c-\operatorname{Ind}_{\Gamma_{\epsilon}}^{J\left(\mathbb{Q}_{p}\right)} H^{\bullet}\left(C_{\epsilon}^{\infty}\right)\right)_{\rho}$ denotes the representation

$$
\sum_{i, j}(-1)^{i+j} \underline{\lim }_{\longrightarrow} U \subseteq U_{0} \operatorname{Ext}_{J\left(\mathbb{Q}_{p}\right)}^{i}\left(c-\operatorname{Ind}_{\Gamma_{\epsilon}}^{J\left(\mathbb{Q}_{p}\right)} H_{c}^{j}\left(C_{\epsilon}^{U} \times_{E_{0}} \hat{\bar{E}}_{0}, \mathbb{Q}_{l}\left(D_{\mathcal{X}}\right)\right), \rho\right) .
$$

Similarly, for the case of the system $\mathcal{M}^{\infty} \times_{\mathcal{M}^{U_{0}}} \mathcal{F}^{U_{0}}$, let us consider the subspace $D=j^{-1}\left(Z^{\text {rig }}\right)$ of $\mathcal{F}^{\text {rig }}$. Again, it is an open subspace, which is stable under the action of $\Gamma \subset J\left(\mathbb{Q}_{p}\right)$ and satisfies the condition $\mathcal{F}^{\text {rig }}=\cup_{t \in J\left(\mathbb{Q}_{p}\right)} D$. For any level $U \subseteq U_{0}$, we write $D^{U}=\mathcal{M}^{U} \times j^{-1}\left(Z^{\text {rig }}\right)$ (and $D_{\epsilon}^{U}=\mathcal{M}^{U} \times j^{-1}\left(Z_{\epsilon}^{\text {rig }}\right)$ ).

Then, for each level $U$, there exists a spectral sequence analogous to the one above computing the $l$-adic cohomology of the rigid analytic space $\mathcal{M}^{U} \times_{\mathcal{M}^{\text {rig }}} \mathcal{F}^{\text {rig }}$ in terms of the cohomology of the subspaces $D_{\epsilon}^{U}$. In particular, there is an equality of virtual admissible representations of $G\left(\mathbb{Q}_{p}\right) \times W_{E}$

$$
H^{\bullet}\left(\mathcal{F}^{\infty}\right)_{\rho}=\sum_{p \geq 1}(-1)^{p} \sum_{\epsilon \in \Gamma \backslash\left(J\left(\mathbb{Q}_{p}\right) / \Gamma\right)_{\neq}^{p}}\left(c-\operatorname{Ind}_{\Gamma_{\epsilon}}^{J\left(\mathbb{Q}_{p}\right)} H^{\bullet}\left(D_{\epsilon}^{\infty}\right)\right)_{\rho}
$$

where, for each $\epsilon,\left(c-\operatorname{Ind}_{\Gamma_{\epsilon}}^{J\left(\mathbb{Q}_{p}\right)} H^{\bullet}\left(D_{\epsilon}^{\infty}\right)\right)_{\rho}$ denotes the representation

$$
\sum_{i, j}(-1)^{i+j} \underline{\lim }_{\longrightarrow \subseteq U_{0}} \operatorname{Ext}_{J\left(\mathbb{Q}_{p}\right)}^{i}\left(c-\operatorname{Ind}_{\Gamma_{\epsilon}}^{J\left(\mathbb{Q}_{p}\right)} H_{c}^{j}\left(D_{\epsilon}^{U} \times_{E_{0}} \hat{\bar{E}}_{0}, \mathbb{Q}_{l}\left(D_{\mathcal{F}}\right)\right), \rho\right) .
$$

We now fix a level $U$, and consider the $\Gamma$-invariant stratification of $Z^{U}$ introduced in Section 9.1. Let $\left\{\mathcal{W}_{\alpha}^{c}\right\}_{\alpha}, \alpha=0, \ldots, r$, denote the filtration of $Z^{U}$ by increasing closed subspaces, associated with the stratification by locally closed $\left\{\mathcal{W}_{\alpha}=\mathcal{M}^{U} \times \mathcal{V}_{\alpha}^{\text {rig }}\right\}_{\alpha}$ (i.e. $Z^{U}=\mathcal{W}_{r}$ and, for all $\alpha, \mathcal{W}_{\alpha} \subset \mathcal{W}_{\alpha}^{c}$ is open and $\left.\mathcal{W}_{\alpha}=\mathcal{W}_{\alpha}^{c}-\mathcal{W}_{\alpha-1}^{c}\right)$. Then, $\mathcal{W}_{\alpha}^{c} \cap \mathcal{X}^{U}$ (resp. $\left\{j^{-1} \mathcal{W}_{\alpha}^{c}\right\}_{\alpha}$ ) is a $\Gamma$-invariant filtration of $C^{U}=Z^{U} \cap \mathcal{X}^{U}$ (resp. $D^{U}=j^{-1} Z^{U}$ ) by increasing closed subspaces, associated with the stratification by locally closed $\mathcal{W}_{\alpha} \cap \mathcal{X}^{U}$ (resp. $\left\{j^{-1} \mathcal{W}_{\alpha}=\mathcal{M}^{U} \times \mathcal{U}_{\alpha}^{\text {rig }}\right\}_{\alpha}$ ). Moreover, for any $p \geq 1$ and $\epsilon \in \Gamma \backslash\left(J\left(\mathbb{Q}_{p}\right) / \Gamma\right)_{\neq}^{p}$, these stratifications give rise to corresponding $\Gamma_{\epsilon}$-invariant stratifications of $C_{\epsilon}^{U}$ (resp. $D_{\epsilon}^{U}$ ), with locally closed strata $\mathcal{W}_{\epsilon, \alpha} \cap \mathcal{X}^{U}$ (resp. $j^{-1} \mathcal{W}_{\epsilon, \alpha}$ ), where $\mathcal{W}_{\epsilon, \alpha}=\mathcal{W}_{\alpha} \cap \epsilon_{1} \mathcal{W}_{\alpha} \cap \cdots \cap \epsilon_{p} \mathcal{W}_{\alpha}$

For any set of indexes $p, \epsilon$ and $\alpha$, the cohomologies with compact support of $\mathcal{W}_{\epsilon, \alpha}^{c} \cap \mathcal{X}^{U}$, $\mathcal{W}_{\epsilon, \alpha-1}^{c} \cap \mathcal{X}^{U}$ and $\mathcal{W}_{\epsilon, \alpha} \cap \mathcal{X}^{U}$ (resp. $j^{-1} \mathcal{W}_{\epsilon, \alpha}^{c}, j^{-1} \mathcal{W}_{\epsilon, \alpha-1}^{c}$ and $j^{-1} \mathcal{W}_{\epsilon, \alpha}$ ) fit in an exact triangle. Therefore, for each $l$-adic admissible representation $\rho$ of $J\left(\mathbb{Q}_{p}\right)$, we obtain the following equalities of virtual representations (with notations analogous to the one introduced above):

$$
\left(c-\operatorname{Ind}_{\Gamma_{\epsilon}}^{J\left(\mathbb{Q}_{p}\right)} H^{\bullet}\left(C_{\epsilon}^{\infty}\right)\right)_{\rho}=\sum_{\alpha}\left(c-\operatorname{Ind}_{\Gamma_{\epsilon}}^{J\left(\mathbb{Q}_{p}\right)} H^{\bullet}\left(\mathcal{W}_{\epsilon, \alpha}^{\infty}\right) \cap \mathcal{X}^{\infty}\right)_{\rho}
$$

and

$$
\left(c-\operatorname{Ind}_{\Gamma_{\epsilon}}^{J\left(\mathbb{Q}_{p}\right)} H^{\bullet}\left(D_{\epsilon}^{\infty}\right)\right)_{\rho}=\sum_{\alpha}\left(c-\operatorname{Ind}_{\Gamma_{\epsilon}}^{J\left(\mathbb{Q}_{p}\right)} H^{\bullet}\left(j^{-1} \mathcal{W}_{\epsilon, \alpha}^{\infty}\right)\right)_{\rho}
$$


Thus, in order to establish the first equality in the statement, it suffices to check that for all indexes $p, \epsilon$ and $\alpha$, the maps

$$
\left(j_{\epsilon, \alpha}^{U}\right)_{!}: R \Gamma_{c}\left(j^{-1}\left(\mathcal{W}_{\epsilon, \alpha}\right) \times_{E_{0}} \hat{\bar{E}}_{0}, \mathbb{Q}_{l}\right) \rightarrow R \Gamma_{c}\left(\left(\mathcal{W}_{\epsilon, \alpha} \cap \mathcal{X}^{U}\right) \times_{E_{0}} \hat{\bar{E}}_{0}, \mathbb{Q}_{l}\right)
$$

are quasi-isomorphisms, and this follows from Part (3) of Theorem 9.2.

We now focus on the second equality. In this case $U=U_{0}$ and we write $C_{\epsilon}=C_{\epsilon}^{U_{0}}$. In order to conclude it suffices to check that the maps on compact supported cohomology, induced by the inclusions $i_{\epsilon}=i_{\mid C_{\epsilon}}: C_{\epsilon} \rightarrow Z_{\epsilon}$ (under Poincarré duality),

$$
\left(i_{\epsilon}^{*}\right)^{\vee}: R \Gamma_{c}\left(\left(C_{\epsilon} \times_{E_{0}} \hat{\bar{E}}_{0}, \mathbb{Q}_{l}\left(D_{\mathcal{X}}\right)\right)\left[2 D_{\mathcal{X}}\right] \rightarrow R \Gamma_{c}\left(Z_{\epsilon} \times_{E_{0}} \hat{\bar{E}}_{0}, \mathbb{Q}_{l}\left(D_{\mathcal{M}}\right)\right)\left[2 D_{\mathcal{M}}\right]\right.
$$

are quasi-isomorphisms, or equivalently that the maps $i_{\epsilon}^{*}$ on cohomology are quasiisomorphisms, for all $\epsilon$.

We recall that we chose the subspace $Z$ of $\mathcal{M}^{U_{0}}=\mathcal{M}^{\text {rig }}$ as the rigid analytic fiber of the formal completion of $\mathcal{M}$ along a closed subscheme $Z_{0}$ of $\mathcal{M}^{\text {red }}$, i.e. $Z=s p_{\mathcal{M}}^{-1}\left(Z_{0}\right)$. Thus, analogous descriptions also hold for the subspaces $Z_{\epsilon}$ and $C_{\epsilon}$. I.e., for all $\epsilon, Z_{\epsilon}=s p_{\mathcal{M}}^{-1}\left(Z_{\epsilon}^{\text {red }}\right)$ and $C_{\epsilon}=s p_{\mathcal{X}}^{-1}\left(C_{\epsilon}^{\text {red }}\right)$, for $Z_{\epsilon}^{\text {red }}$ (resp. $\left.C_{\epsilon}^{\text {red }}\right)$ the closed $k$-subschemes of $\mathcal{M}^{\text {red }}$ (resp. $\mathcal{X}^{\text {red }}$ ), defined as $Z_{\epsilon}^{\text {red }}=Z_{0} \cap \epsilon_{1} Z_{0} \cap \cdots \cap \epsilon_{p} Z_{0}$ (resp. $\left.C_{\epsilon}^{\text {red }}=Z_{\epsilon}^{\text {red }} \cap \mathcal{X}^{\text {red }}\right)$ ). In particular, the $Z_{\epsilon}^{\text {red }}$ 's and the $C_{\epsilon}^{\text {red's }}$ are projective $k$-schemes since such are all the irreducible components of $\mathcal{M}^{\text {red }}$ ([31], Theorem 2.32, p. 63) and thus also those of $\mathcal{X}^{\text {red }}$. Therefore, we may apply Berkovich's theory of vanishing cycles for formal schemes ([3], Corollary 2.5, p. 373) to compute the $l$-adic cohomology of $Z_{\epsilon}$ and $C_{\epsilon}$, in terms of the cohomology of their reduced fiber, with coefficient in the vanishing cycle sheaves of $\mathbb{Q}_{l}$. Since $Z_{\epsilon}=s p_{\mathcal{M}}^{-1}\left(Z_{\epsilon}^{\text {red }}\right)$ (resp. $C_{\epsilon}=s p_{\mathcal{X}}^{-1}\left(C_{\epsilon}^{\text {red }}\right)$ ), the vanishing cycles sheaves of $\mathbb{Q}_{l}$ for $Z_{\epsilon}$ (resp. $C_{\epsilon}$ ) are simply the restrictions of the vanishing cycles sheaves of $\mathbb{Q}_{l}$ for $\mathcal{M}$ (resp. $\mathcal{X}$ ). Therefore, we have

$$
R \Gamma\left(Z_{\epsilon} \times_{E_{0}} \hat{\bar{E}}_{0}, \mathbb{Q}_{l}\right) \simeq R \Gamma\left(Z_{\epsilon}^{\text {red }}, R \Psi_{\eta}^{\mathcal{M}}\left(\mathbb{Q}_{l}\right)_{\mid Z_{\epsilon}^{\text {red }}}\right)
$$

and

$$
R \Gamma\left(C_{\epsilon} \times_{E_{0}} \hat{\bar{E}}_{0}, \mathbb{Q}_{l}\right) \simeq R \Gamma\left(C_{\epsilon}^{\mathrm{red}}, R \Psi_{\eta}^{\mathcal{X}}\left(\mathbb{Q}_{l}\right)_{\mid X_{\epsilon}^{\mathrm{red}}}\right) .
$$

In the case of level $U_{0}$, the formal smoothness of $\mathcal{M}$ implies that the vanishing cycle sheaves $R^{q} \Psi_{\eta}^{\mathcal{M}}\left(\mathbb{Q}_{l}\right)$ over $\mathcal{M}^{\text {red }}$ are trivial, i.e. $R^{0} \Psi_{\eta}^{\mathcal{M}}\left(\mathbb{Q}_{l}\right)=\mathbb{Q}_{l}$, and $R^{q} \Psi_{\eta}^{\mathcal{M}}\left(\mathbb{Q}_{l}\right)=0$, for $q>0$. Therefore, for all index $q \geq 0$, we have

$$
H_{c}^{2 D_{\mathcal{M}}-q}\left(Z_{\epsilon} \times_{E_{0}} \hat{\bar{E}}_{0}, \mathbb{Q}_{l}\left(D_{\mathcal{M}}\right)\right) \simeq H^{q}\left(Z_{\epsilon} \times_{E_{0}} \hat{\bar{E}}_{0}, \mathbb{Q}_{l}\right)^{*} \simeq H^{q}\left(Z_{\epsilon}^{\mathrm{red}}, \mathbb{Q}_{l}\right)^{*} .
$$

Similarly, it follows from Property (3) in Theorem 9.2 that for each $x \in \mathcal{X}^{\text {red }}$

$$
R^{q} \Psi_{\eta}^{\mathcal{X}}\left(\mathbb{Q}_{l}\right)_{x} \simeq R^{q} \Psi_{\eta}^{\mathcal{F}}\left(\mathbb{Q}_{l}\right)_{x}
$$

Since $\mathcal{F}$ is formal smooth this implies that the vanishing cycles sheaves of $\mathbb{Q}_{l}$ for $\mathcal{X}$ are also trivial, i.e. $R^{0} \Psi_{\eta}^{\mathcal{X}}\left(\mathbb{Q}_{l}\right)=\mathbb{Q}_{l}$, and $R^{q} \Psi_{\eta}^{\mathcal{X}}\left(\mathbb{Q}_{l}\right)=0$, for $q>0$. Therefore, for all $q \geq 0$, we have

$$
H_{c}^{2 D_{\mathcal{X}}-q}\left(C_{\epsilon} \times_{E_{0}} \hat{\bar{E}}_{0}, \mathbb{Q}_{l}\left(D_{\mathcal{X}}\right)\right) \simeq H^{q}\left(C_{\epsilon} \times_{E_{0}} \hat{\bar{E}}_{0}, \mathbb{Q}_{l}\right)^{*} \simeq H^{q}\left(C_{\epsilon}^{\mathrm{red}}, \mathbb{Q}_{l}\right)^{*}
$$

Finally, Property (1) in Theorem 9.2 implies that $Z_{\epsilon}^{\text {red }}=C_{\epsilon}^{\text {red }}$. Therefore, the induced morphism $i_{\epsilon}^{\text {red }}: C_{\epsilon}^{\text {red }} \rightarrow Z_{\epsilon}^{\text {red }}$ is simply the identity, and we conclude. 
9.3. - Before presenting the construction of the space $\mathcal{X}$, we recall Rapoport's and Zink's period morphism ([31], Chapter 5).

Let $\mathcal{G}$ denote the universal Barsotti-Tate group over $\mathcal{M}$, together with a quasi-isogeny $\beta: \Sigma_{\mathcal{M}_{0}} \rightarrow \mathcal{G}_{\mathcal{M}_{0}}$, defined over $\mathcal{M}_{0}=Z(p) \subset \mathcal{M}$.

We denote by $M_{\mathcal{G}}$ the Lie algebra of the universal extension of $\mathcal{G}$ (i.e. the crystal of $\mathcal{G}$ in the case when we may apply the theory of Grothendieck-Messing), by $\mathcal{L}_{\mathcal{G}}=\operatorname{Lie}(\mathcal{G})$ the Lie algebra of $\mathcal{G}$, and by $\pi_{\mathcal{G}}: M_{\mathcal{G}} \rightarrow \mathcal{L}_{\mathcal{G}}$ the natural epimorphism of locally free $\mathcal{O}_{\mathcal{M}}$-modules.

Then, the quasi-isogeny $\beta$ induces a canonical isomorphism of locally free $\mathcal{O}_{\mathcal{M}^{\text {rig }}}$-modules of finite rank,

$$
N(V) \otimes_{E_{0}} \mathcal{O}_{\mathcal{M}^{\mathrm{rig}}}=\left(M_{\Sigma} \otimes_{\mathcal{O}_{E_{0}}} E_{0}\right) \otimes_{E_{0}} \mathcal{O}_{\mathcal{M}^{\mathrm{rig}}} \simeq M_{\mathcal{G}}^{\mathrm{rig}}=M_{\mathcal{G}} \otimes_{\mathcal{O}_{\mathcal{M}}} \mathcal{O}_{\mathcal{M}^{\mathrm{rig}}}
$$

(see [31], Proposition 5.15). In particular, we may regard $\mathcal{L}_{\mathcal{G}}$ as a quotient of $V=N(V)$,

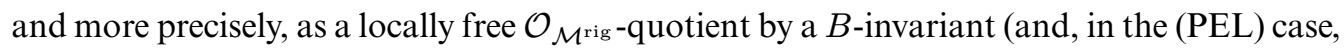
totally isotropic) subspace of $V$ in the isomorphism class of $V_{1}$, the weight 1 subspace of $V$ (see Section 2).

Equivalently, let $\left.\mathcal{G} r=\mathcal{G} r\left(V, V_{0}\right)\right)=\mathcal{G} r(G, \mu)$ denote the Grassmanian variety over $E$ parameterizing the $B$-invariant (and, in the (PEL) case, totally isotropic) subspaces of $V$ in the isomorphism class of $V_{1}$ (see [31], Chapter 1). Then, the above construction describes a morphism

$$
\pi: \mathcal{M}^{\mathrm{rig}} \rightarrow \mathcal{G} r \otimes_{E} E_{0}
$$

which is called (the first component of) the period morphism of the moduli problem. It follows immediately from the construction that $\pi$ is $J\left(\mathbb{Q}_{p}\right)$-equivariant (for the natural action of $J\left(\mathbb{Q}_{p}\right)=\operatorname{Aut}(N(V)) \subset G L(V)$ on $G r$ ) and moreover it is étale (see [31], Proposition 5.17).

It is a consequence of Fontaine's theory (see [9], [5]) that the image of the period morphism is the (weakly) admissible open subspace $\mathcal{G} r^{w a}$ of $\mathcal{G} r$ (see [31], Sections 1.31 and ff.).

9.4. - We now consider a decomposition of $V, V=\oplus_{i} V^{i}$, as in Section 3.2, and write $\left(n_{1}, \ldots, n_{t}\right)$ for the associated partition of $r$, the number of isoclinic components of $V$. I.e., for each $i, V^{i}=\oplus_{n_{i-1}+1}^{n_{i}} W^{s}$, for $V=\oplus_{s} W^{s}$ the slope decomposition.

We also write $V_{\bullet}$ for the associated filtration of $V$, as in Section 4.1. For $\epsilon=0,1$, we write $V_{\epsilon}^{i}$ (resp. $V_{\epsilon, j}$ ) the weight decomposition on $V_{K}^{i}$ (resp. $V_{j, K}$ ) induced from the weight decomposition on $V_{K}$.

To any point $x$ of $\mathcal{G} r$, corresponding to a quotient $V \rightarrow \mathcal{L}$, we associate the filtration on $\mathcal{L}, \mathcal{L}_{\bullet}$, induced from the filtration $V_{\bullet}$ on $V$, and its associated graded pieces, $\mathcal{L}^{i}(i=1, \ldots, t)$.

The following conditions of Schubert type

$$
\text { for all } i: r k\left(\mathcal{L}^{i}\right) \geq d_{i}=\sum_{n_{i-1}+1}^{n_{i}} \lambda_{s} h_{s},
$$

for $\lambda_{s}$ and $h_{s}$, respectively the slope of $W^{s}$ and its dimension, define a closed subscheme of $\mathcal{G} r$ (called the Schubert cell), which we denote by $\mathcal{S}$.

We remark that $\mathcal{S}$ is stable under the action of $J\left(\mathbb{Q}_{p}\right)$ on $\mathcal{G} r$. Moreover, by comparing the above inequalities with the ones defining the weakly admissible subspace $\mathcal{G} r^{w a}$ of $\mathcal{G} r$ (see [31], 
p. 30), it is immediate to realize that the proper closed subset of the Schubert cell $\mathcal{S}$ which is defined by strict inequalities, does not intersect $\mathcal{G} r^{w a} \subset \mathcal{G} r$.

We define $\mathcal{S}^{\prime}$ to be the closed subset of $\mathcal{S}$ parameterizing the $B$-invariant (and, in the (PEL) case, totally isotropic) filtered subspaces of $\left(V, V_{\bullet}\right)$ in the isomorphism class of $\left(V_{1}, V_{1, \bullet}\right)$. Then, $\mathcal{S}^{\prime}$ is closed in $\mathcal{G} r$ and stable under the action of $J\left(\mathbb{Q}_{p}\right)$. Moreover, it follows immediately from the construction that the image of $\mathcal{F}$, under the composition of the period morphism with $j: \mathcal{F} \rightarrow \mathcal{M}$, is $\mathcal{S}^{\prime} \cap \mathcal{G} r^{w a} \subset \mathcal{G} r^{w a}$.

We define $X=\pi^{-1} \mathcal{S}^{\prime} \subset \mathcal{M}^{\text {rig }}$. Then, $X$ is a smooth closed rigid analytic subspace of $\mathcal{M}^{\text {rig }}$, and clearly the morphism $j^{\text {rig }}: \mathcal{F}^{\text {rig }} \rightarrow \mathcal{M}^{\text {rig }}$ factors via the inclusion $X \subset \mathcal{M}^{\text {rig }}$. Furthermore, for $\left\{\mathcal{V}_{\alpha}\right\}_{\alpha}$ a stratification of $\mathcal{M}$ defined as in Section 9.1 (and $\left.\mathcal{U}_{\alpha}=j^{-1}\left(\mathcal{V}_{\alpha}\right) \subset \mathcal{F}\right)$, it follows from the construction that

$$
j_{\alpha}\left(\mathcal{U}_{\alpha}\right)^{\text {rig }}=X \cap \mathcal{V}_{\alpha}^{\text {rig }}, \text { for all } \alpha .
$$

Lemma 9.4. - There exists a (unique) closed formal subscheme, formally locally of finite type,

$$
i: \mathcal{X} \hookrightarrow \mathcal{M}
$$

defined over $\mathcal{O}_{E_{0}}$, which is invariant under the action of $J\left(\mathbb{Q}_{p}\right)$ and the descent data to $E$, and such that

1. $\mathcal{X}^{\text {rig }}=X \subset \mathcal{M}^{\text {rig }}$

2. $\mathcal{X} \cap \mathcal{V}_{\alpha}=j_{\alpha}\left(\mathcal{U}_{\alpha}\right)$, for all $\alpha$.

Proof. - For $Z$ as in Section 9.1 (i.e. $\mathcal{M}=\cup_{t \in J\left(\mathbb{Q}_{p}\right) / \Gamma} Z$ and $Z=\coprod_{\alpha} \mathcal{V}_{\alpha}$ ), we construct $\mathcal{X}$ as $\mathcal{X}=\cup_{t \in J\left(\mathbb{Q}_{p}\right) / \Gamma} \mathcal{X}_{Z}$, for $\mathcal{X}_{Z} \subset Z$ the unique closed formal subscheme of $Z$ satisfying the conditions $\mathcal{X}_{Z}^{\text {rig }}=X \cap Z^{\text {rig }} \subset Z^{\text {rig }}$, and $\mathcal{X} \cap \mathcal{V}_{\alpha}=j_{\alpha}\left(\mathcal{U}_{\alpha}\right)$, for all $\alpha$. The existence of a closed formal subscheme $\mathcal{X}_{Z}$ of $Z$, with the above properties, is due to a result of De Jong on rigid descent for closed formal subschemes ([14], Proposition 7.5.2). More precisely, we apply De Jong's result to the data $\left(\left\{j_{\alpha}\left(\mathcal{U}_{\alpha}\right)\right\}_{\alpha}, X \cap Z^{\text {rig }}\right)$, where, for each $\alpha$, we regard the formal scheme $j_{\alpha}\left(\mathcal{U}_{\alpha}\right)$ as a closed formal subscheme of $\mathcal{V}_{\alpha}$, after Lemma 9.1.

It is an easy remark that all the remaining properties of $\mathcal{X}$ stated in Theorem 9.2 are an immediate consequence of the above construction of $\mathcal{X}$. In particular, the equality $\mathcal{X}^{\text {red }}=$ $\mathcal{M}^{\text {red }}$ follows from the equalities $j_{\alpha}^{\text {red }}\left(\mathcal{U}_{\alpha}^{\text {red }}\right)=\mathcal{V}_{\alpha}^{\text {red }}$, for all $\alpha$, in Lemma 9.1.

\section{Forgetting the filtration: the case of $(\mathrm{HN})$ type}

In this section, we restrict our discussion to the cases when the admissible pair $(b, \mu)$ is of (HN) type, $M \supseteq M_{b}$ and $P \supseteq P_{b}$. Our analysis strongly relays on properties specific to the filtrations which are coarser than or equal to the Hodge-Newton filtration.

Under these hypotheses, we establish the variant of Harris' conjecture discussed in the introduction, Section 1.3. More precisely, we establish the following result. 
Theorem 10.1. - Assume the admissible pair is of (HN) type, $M \supseteq M_{b}$ and $P \supseteq P_{b}$.

Then, for any $l$-adic admissible representation $\rho$ of $J\left(\mathbb{Q}_{p}\right)$, we have an equality of virtual admissible representations of $G\left(\mathbb{Q}_{p}\right) \times W_{E}$ :

$$
H^{\bullet}\left(\mathcal{M}^{\infty}\right)_{\rho}=\operatorname{Ind}_{P\left(\mathbb{Q}_{p}\right)}^{G\left(\mathbb{Q}_{p}\right)} H^{\bullet}\left(\mathcal{P}_{M}^{\infty}\right)_{\rho}
$$

This results implies in particular that the cohomology of the tower of Rapoport-Zink spaces associated with a pair $(b, \mu)$ of $(\mathrm{HN})$ type contains no supercuspidal representations of $G\left(\mathbb{Q}_{p}\right)$.

In the case when all break-points of $\nu_{b}$ are of (HN) type, $M_{b}=L_{b}$, and the corresponding Rapoport-Zink spaces $\mathcal{P}_{L_{b}}$ are basic. Thus, in this case, the above theorem reduces the computation of the cohomology of non-basic Rapoport-Zink spaces to that of the associated basic ones.

Lemma 10.2. - Maintaining the notations of Section 9.1.

Assume that the admissible pair $(b, \mu)$ is of $(H N)$ type, $M \supseteq M_{b}$ and $P \supseteq P_{b}$.

Then, for all indexes $\alpha$, the corresponding restriction of the map $j, j_{\alpha}: \mathcal{U}_{\alpha}=j^{-1} \mathcal{V}_{\alpha} \rightarrow \mathcal{V}_{\alpha}$, are isomorphisms.

Equivalently, with the notations of Theorem 9.2, $\mathcal{X}=\mathcal{X}_{P}=\mathcal{M}$.

Proof. - Since we already know that the $j_{\alpha}$ are closed embeddings, it suffices to check that they are bijective on points. Let $x$ be a point of $\mathcal{M}$. Then, $x$ corresponds to the data of a Barsotti-Tate group $\mathcal{G}$ with additional structures, defined over a complete local ring $R$, with residue field $k$, and an isogeny $\beta: \Sigma_{k} \rightarrow G=\mathcal{G}_{k}$. Under our hypotheses, Theorem 6.3 implies that $\mathcal{G} / R$ admits a unique filtration $\mathcal{G}_{\bullet}$, which is canonically split over $k, G=\oplus_{i} G^{i}$, associated with the parabolic subgroup $P$. (This is because we chose $P \supseteq P_{b}$, where $P_{b}$ is the parabolic subgroup associated with the Hodge-Newton filtration on $N_{b}(V)$.) For any $i=1, \ldots, t$, the constituent $\Sigma^{i}$ of $\Sigma$ maps surjectively onto the corresponding constituent $G^{i}$ of $G$ and trivially to the others. (This is because the decomposition is compatible with the slope filtration.) Thus, the isogeny $\beta: \Sigma \rightarrow G$ is of the form $\beta=\oplus_{i} \beta^{i}$, for some isogenies $\beta^{i}: \Sigma^{i} \rightarrow G^{i}, i=1, \ldots, t$. Thus, the data $(\mathcal{G}, \mathcal{G} \bullet, \beta)$ defines a (unique) point $x^{\prime}$ of $\mathcal{F}=\mathcal{F}_{P}$ mapping to $x$.

Theorem 10.3. - Assume the admissible pair $(b, \mu)$ is of (HN) type, $M \supseteq M_{b}$ and $P \supseteq P_{b}$.

Then, for any $l$-adic admissible representation $\rho$ of $J\left(\mathbb{Q}_{p}\right)$, we have an equality of virtual admissible representations of $G\left(\mathbb{Q}_{p}\right) \times W_{E}$ :

$$
H^{\bullet}\left(\mathcal{M}^{\infty}\right)_{\rho}=H^{\bullet}\left(\mathcal{M}^{\infty} \times{ }_{\mathcal{M}^{U_{0}}} \mathcal{F}_{P}^{U_{0}}\right)_{\rho} .
$$

Proof. - We are simply restating Theorem 9.3 using the result of Lemma 10.2.

The above theorem combined with Theorems 7.1 and 8.1 implies Theorem 10.1. 


\section{Application to the study of the cohomology of Shimura varieties}

We conclude our discussion with an application of Theorem 10.1 to the study of the $l$ adic cohomology of certain Shimura varieties. More precisely, in [23] (Theorem 22), we established an explicit formula expressing the $l$-adic cohomology of certain Shimura varieties in terms of the cohomology of the corresponding Rapoport-Zink spaces and of some other varieties, the Igusa varieties. Using those formulas, it is possible to translate Theorem 10.1 into information about the absence of supercuspidal representations inside some specified subrepresentations of the $l$-adic cohomology of certain Shimura varieties. (In the following, we recall very briefly the context and pertinent results and refer to [23] for precise definitions and statements.)

11.1. - Let $\mathcal{S}^{\infty}$ be a tower of (PEL)-type Shimura varieties, associated to a Shimura datum $(G, h)$ over $\mathbb{Q}$ which is unramified at $p$. These are smooth quasi-projective varieties defined over a number field $E$, called the reflex field, which is unramified at all primes $v$ above $p$. Let $v$ be such a prime; we denote by $E_{v}$ (resp. $\mathcal{O}_{E_{v}}, k_{v}$ and $\bar{E}_{v}$ ) the completion of $E$ at $v$ (resp. its ring of integers, its residue field and the completion of an algebraic closure of $E_{v}$ ), and by $\overline{\mathcal{S}}$ the special fibers at $v$ of the Shimura varieties $\mathcal{S}$.

There exists a natural stratification of $\overline{\mathcal{S}}$, by locally closed reduced subschemes, $\left\{\overline{\mathcal{S}}_{b}\right\}_{b}$, called the Newton polygon stratification, which is indexed by the elements $b \in B\left(G_{\mathbb{Q}_{p}}, \mu_{h}\right)$ (for $G_{\mathbb{Q}_{p}}$ the restriction of $G$ over $\mathbb{Q}_{p}$ and $\mu_{h}$ the conjugacy class of cocharacters of $G_{\mathbb{Q}_{p}}$ corresponding to $h$ ) and is stable under the action of $G\left(\mathbb{A}_{f}\right)$ (for $\mathbb{A}_{f}$ the finite adeles of $\mathbb{Q}$ ). Namely, for any $b \in B\left(G_{\mathbb{Q}_{p}}, \mu_{h}\right)$ (regarded as an isogeny class of Barsotti-Tate groups with additional structures), the associated stratum is defined by

$$
\overline{\mathcal{S}}_{b}=\left\{x \in \overline{\mathcal{S}} \mid A_{x}\left[p^{\infty}\right] \in b\right\} .
$$

Conjecturally, the Newton polygon strata are all non-empty. In the case of $b \mu$-ordinary the corresponding stratum is known to be open and dense ([32], Theorem 1.6.2, p. 584).

Let $R^{q} \Psi_{\eta}\left(\mathbb{Q}_{l}\right), q \geq 0$, denote the $l$-adic vanishing cycles of the Shimura varieties $\mathcal{S}$, and for each $b \in B\left(G_{\mathbb{Q}_{p}}, \mu_{h}\right)$ consider the virtual representation of $G\left(\mathbb{A}_{f}\right) \times W_{E_{v}}$

$$
H_{c}^{\bullet}\left(\overline{\mathcal{S}}_{b}^{\infty}, R^{\bullet} \Psi_{\eta}\left(\mathbb{Q}_{l}\right)\right)=\sum_{p, q \geq 0}(-1)^{p+q} H_{c}^{p}\left(\overline{\mathcal{S}}_{b}^{\infty}, R^{q} \Psi_{\eta}\left(\mathbb{Q}_{l}\right)\right)
$$

In the case when the Shimura varieties are proper, their $l$-adic cohomology is naturally the sum of the above representations, as $b$ varies. I.e., there is an equality of virtual representations of $G\left(\mathbb{A}_{f}\right) \times W_{E_{v}}$

$$
\sum_{i}(-1)^{i} H^{i}\left(\mathcal{S}^{\infty} \times_{E} \bar{E}_{v}, \mathbb{Q}_{l}\right)=\sum_{b \in B\left(G_{\mathbb{Q}_{p}}, \mu_{h}\right)} H_{c}^{\bullet}\left(\overline{\mathcal{S}}_{b}^{\infty}, R^{\bullet} \Psi_{\eta}\left(\mathbb{Q}_{l}\right)\right) \quad(\mathcal{S} \text { proper }) .
$$

In [23], we showed how these representations associated with the Newton polygon stratification of the Shimura varieties can be computed in terms of the $l$-adic cohomology of the corresponding Rapoport-Zink spaces and Igusa varieties. In particular, via those formulas, Theorem 10.1 implies the following result.

$4^{\mathrm{e}}$ SÉRIE - TOME $41-2008-\mathrm{N}^{\mathrm{o}} 5$ 
COROLlaRY 11.1. - Let $b \in B\left(G_{\mathbb{Q}_{p}}, \mu_{h}\right)$ be an element of $(H N)$ type.

Then, the virtual representation $H_{c}^{\bullet}\left(\overline{\mathcal{S}}_{b}^{\infty}, R^{\bullet} \Psi_{\eta}\left(\mathbb{Q}_{l}\right)\right)$ contains no supercuspidal representations of $G\left(\mathbb{Q}_{p}\right)$.

\section{REFERENCES}

[1] V. G. Berkovich, Étale cohomology for non-Archimedean analytic spaces, Publ. Math. I.H.É.S. 78 (1993), 5-161.

[2] V. G. Ber Kovich, Vanishing cycles for formal schemes, Invent. Math. 115 (1994), 539571.

[3] V. G. Berkovich, Vanishing cycles for formal schemes. II, Invent. Math. 125 (1996), 367-390.

[4] P. Boyer, Mauvaise réduction des variétés de Drinfeld et correspondance de Langlands locale, Invent. Math. 138 (1999), 573-629.

[5] P. Colmez, J.-M. Fontaine, Construction des représentations $p$-adiques semi-stables, Invent. Math. 140 (2000), 1-43.

[6] M. Demazure, Lectures on p-divisible groups, Lecture Notes in Math. 302, Springer, 1972.

[7] V. G. Drinfel'D, Coverings of $p$-adic symmetric domains, Funkcional. Anal. i Priložen. 10 (1976), 29-40.

[8] L. Fargues, Cohomologie des espaces de modules de groupes $p$-divisibles et correspondances de Langlands locales, Astérisque 291 (2004), 1-199.

[9] J.-M. Fontaine, Modules galoisiens, modules filtrés et anneaux de Barsotti-Tate, in Journées de Géométrie Algébrique de Rennes (Rennes, 1978), Vol. III, Astérisque 65, Soc. Math. France, 1979, 3-80.

[10] A. Grothendieck, Groupes de Barsotti-Tate et cristaux de Dieudonné, Séminaire de Mathématiques Supérieures, No. 45 (Été 1970), Presses de l’Univ. Montréal, 1974.

[11] M. Harris, Local Langlands correspondences and vanishing cycles on Shimura varieties, in European Congress of Mathematics, Vol. I (Barcelona, 2000), Progr. Math. 201, Birkhäuser, 2001, 407-427.

[12] M. HARris, R. TAYLOR, The geometry and cohomology of some simple Shimura varieties, Annals of Math. Studies 151, Princeton University Press, 2001.

[13] L. Illusie, Déformations de groupes de Barsotti-Tate (d'après A. Grothendieck), Astérisque 127 (1985), 151-198.

[14] A. J. D. Jong, Crystalline Dieudonné module theory via formal and rigid geometry, Publ. Math. I.H.É.S. 82 (1995), 5-96.

[15] N. M. Katz, Slope filtration of F-crystals, in Journées de Géométrie Algébrique de Rennes (Rennes, 1978), Vol. I, Astérisque 63, Soc. Math. France, 1979, 113-163.

[16] N. M. Katz, B. Mazur, Arithmetic moduli of elliptic curves, Annals of Math. Studies 108, Princeton University Press, 1985.

[17] R. E. Kоттwitz, Isocrystals with additional structure, Compositio Math. 56 (1985), 201-220. 
[18] R. E. Koтtwitz, Points on some Shimura varieties over finite fields, J. Amer. Math. Soc. 5 (1992), 373-444.

[19] R. E. Kottwitz, Isocrystals with additional structure. II, Compositio Math. 109 (1997), 255-339.

[20] R. E. Koтtwitz, On the Hodge-Newton decomposition for split groups, Int. Math. Res. Not. 26 (2003), 1433-1447.

[21] J. I. Manin, Theory of commutative formal groups over fields of finite characteristic, Uspehi Mat. Nauk 18 (1963), 3-90; Russ. Math. Surveys 18 (1963), 1-80.

[22] E. Mantovan, On certain unitary group Shimura varieties, Astérisque 291 (2004), 201331.

[23] E. Mantovan, On the cohomology of certain PEL-type Shimura varieties, Duke Math. J. 129 (2005), 573-610.

[24] E. Mantovan, E. Viehmann, On the Hodge-Newton filtration for $p$-divisible $\mathcal{O}$-modules, preprint, arXiv:0710.4194, to appear in Math. Z.

[25] W. Messing, The crystals associated to Barsotti-Tate groups: with applications to abelian schemes, Lecture Notes in Math. 264, Springer, 1972.

[26] B. Moonen, Serre-Tate theory for moduli spaces of PEL type, Ann. Sci. École Norm. Sup. 37 (2004), 223-269.

[27] D. Mumford, Lectures on curves on an algebraic surface, Annals of Math. Studies, No. 59, Princeton University Press, 1966.

[28] F. Oort, Newton polygon strata in the moduli space of abelian varieties, in Moduli of abelian varieties (Texel Island, 1999), Progr. Math. 195, Birkhäuser, 2001, 417-440.

[29] F. Oort, T. ZINK, Families of $p$-divisible groups with constant Newton polygon, Doc. Math. 7 (2002), 183-201.

[30] M. Rapoport, M. Richartz, On the classification and specialization of $F$-isocrystals with additional structure, Compositio Math. 103 (1996), 153-181.

[31] M. Rapoport, T. ZINK, Period spaces for p-divisible groups, Annals of Mathematics Studies 141, Princeton University Press, 1996.

[32] T. Wedhorn, Ordinariness in good reductions of Shimura varieties of PEL-type, Ann. Sci. École Norm. Sup. 32 (1999), 575-618.

[33] T. ZINK, On the slope filtration, Duke Math. J. 109 (2001), 79-95.

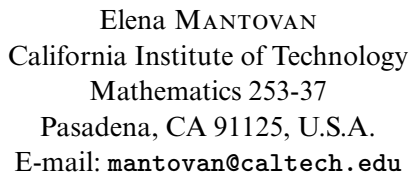

$4^{\text {e }}$ SÉRIE - TOME $41-2008-\mathrm{N}^{\mathrm{o}} 5$ 